\title{
Investigation of dark matter and modified Newtonian dynamics in early-type galaxies through globular cluster systems
}

\author{
S. Samurović
}

\author{
Astronomical Observatory, Volgina 7, 11060 Belgrade, Serbia \\ e-mail: srdjan@aob.bg.ac.rs
}

Received 12 March 2013 / Accepted 31 July 2014

\section{ABSTRACT}

\begin{abstract}
Context. The departures from Newtonian dynamics based on the mass-follows-light approach discovered in the outer parts of some early-type galaxies imply the existence of dark matter and/or necessary modifications to the Newtonian approach. We study dynamical models of a sample of ten early-type galaxies in both Newtonian and MOND approaches.

Aims. The measurements of the radial velocities of the globular clusters in ten massive early-type galaxies are used to test the predictions of dynamical models with and without dark matter assuming Newtonian and MOND approaches out to several effective radii.

Methods. The globular clusters taken from the SLUGGS database are used as tracers of the gravitational potential of the galaxies in a sample. We solve the Jeans equation for both the Newtonian (mass-follows-light and dark matter models) and the MOND approaches by assuming spherical symmetry and compare the resulting mass-to-light ratios with stellar population synthesis models. For both approaches, we apply various assumptions on velocity anisotropy.

Results. We find that the Newtonian mass-follows-light models without a significant amount of dark matter can provide successful fits for only one galaxy (NGC 2768), and for the remaining nine early-type galaxies, various amounts of dark matter are required in the outer parts beyond $2-3 R_{\mathrm{e}}$. With MOND models, we find that four early-type galaxies could be fit without dark matter and that the remaining six galaxies require an additional dark component to successfully fit the line-of-sight observed velocity dispersions; the galaxy NGC 4486 (M 87) is the only galaxy for which dark matter is required in the inner regions, and MOND cannot fit the data without additional dark matter. In the inner region, the galaxy NGC 4365 requires higher mass-to-light ratios than the stellar values from population synthesis, but a reasonable mass-to-light ratio can be reached for MOND assuming slightly tangential orbits. The ten galaxies can be split into two classes: those with concentrations at (NGC 1407) or above the $\Lambda$ CDM concentration-mass relation, given their measured virial masses, and those below this relation. The former generally require dark matter in both Newtonian and MOND approaches, while the latter do not require appreciable amounts of dark matter.
\end{abstract}

Key words. gravitation - galaxies: elliptical and lenticular, $\mathrm{cD}$ - galaxies: kinematics and dynamics - galaxies: halos

\section{Introduction}

The problem of the existence and distribution of dark matter (DM) in early-type (elliptical and lenticular) galaxies remains poorly understood, and the studies of galaxies performed so far show that various possibilities are present: whereas some galaxies, such as NGC 5128 appear to lack DM, or show that DM is not dynamically important out to approximately six effective radii $\left(R_{\mathrm{e}}\right.$; see Samurović 2010 and also Łokas 2008, who confirmed the dearth of DM with a larger mass-to-light ratio) in some cases, it appears that DM is important even in the inner regions. For example, Rix et al. (1997) found that half of the mass within one effective radius in NGC 2434 is dark. Samurović (2012) recently analyzed the massive elliptical galaxy NGC 4472 and found that beyond $\sim 2 R_{\mathrm{e}}$ either DM or modifications of Newtonian dynamics are necessary to successfully fit the observed velocity dispersion. Therefore, since the number of studied galaxies is still small, it is important to analyze as many galaxies as possible to establish the importance of DM in early-type galaxies in detail.

There are several reasons why early-type galaxies are studied to a lesser degree than their spiral counterparts and why the study of early-types is both observationally and theoretically more demanding (see for example the review in Samurović 2007): (i) there is a lack of cool gas in early-types necessary for obtaining rotation curves; (ii) early-type galaxies are faint in their outer parts, which require long exposures to obtain high quality spectra; and (iii) there is no accurate knowledge of the shape of orbits in these galaxies, which leads to a well-known problem of the mass-anisotropy degeneracy (Binney \& Mamon 1982; Tonry 1983). In the study of these galaxies, one may use X-ray halos, but not all early-type galaxies possess it (e.g. IC 3370, see Samurović \& Danziger 2005) and the underlying assumptions about the hot gas are still under discussion (Diehl \& Statler 2007; Humphrey et al. 2011). Humphrey et al. (2006) studied a sample of seven early-type galaxies using Chandra observations and concluded that there is substantial DM in all objects beyond one or several effective radii. Fortunately, the use of various tracers of mass, such as globular clusters (GCs) and planetary nebulae (PNe), allows us to investigate early-type galaxies out to several effective radii and to even assess to a certain degree the presence of anisotropies in their orbits: the example of the detailed study of DM based on GCs in NGC 1407 and its group can be found in Romanowsky et al. (2009) and the example of the use of PNe for the purpose of the study of DM in NGC 4494 is in Napolitano et al. (2009). Recently, Deason et al. (2012), hereafter D12, studied a sample of 15 elliptical galaxies out to $\sim 5 R_{\mathrm{e}}$ using both PNe and GCs and found that, the galaxies that they studied are generally dominated by DM in their outer parts (beyond $\sim 2 R_{\mathrm{e}}$ ). Another example of a detailed study of DM in an 
early-type galaxy out to $\sim 5 R_{\mathrm{e}}$ can be found in Napolitano et al. (2011): they studied NGC 4374 and found that PNe dynamics is consistent with a standard DM halo for the first time.

Gravitation lensing is another valuable tool in the investigation of DM in early-type galaxies. Weak lensing studies (e.g. Kleinheinrich et al. 2006) were used to analyze singular isothermal spheres and Navarro-Frenk-White (Navarro et al. 1997) profiles for a sample of lenses at redshift $z=0.2-0.7$; it was found that early-type galaxies have 2-3 times more massive halos than their late-type counterparts at the same luminosity. Several surveys dedicated to the study of strong lensing, such as SLACS (Auger et al. 2009) and COSMOS (Faure et al. 2008), provided the information required to compare the early-type galaxies at intermediate redshifts. For example, Tortora et al. (2010) analyzed the correlations among central DM content of early-type galaxies, their sizes, and their ages, using a sample of gravitational lenses from the SLACS survey at $z \sim 0.2$, and compared them to a larger sample of $z \sim 0$ galaxies: for a given stellar mass, they found that, the DM fractions increase and the mean densities decrease, concluding that their results agree with studies based on local galaxies.

The study of the constraints from satellite kinematics can provide insight on the mass content of early-type galaxies. Conroy et al. (2007) used the observational data from the DEEP2 Galaxy Redshift Survey and the Sloan Digital Sky Survey (SDSS), studied the motions of satellite galaxies around isolated galaxies, and found that virial-to-stellar mass ratio for red host galaxies with stellar masses $M_{*} \sim 10^{11} h^{-2} M_{\odot}$ to experience an increase of factor $\sim 3$ between redshift $z \sim 1$ and $z \sim 0$ in the virial-to-stellar mass. More et al. (2011) used the kinematics of satellite galaxies from the SDSS catalog to infer the scaling relations between halo mass and central galaxy properties and reached the conclusion that stellar mass is a better indicator of halo mass than luminosity; using a stacking procedure due to a small number of satellite galaxies around individual galaxies, they concluded that more luminous (more massive) central galaxies are found in more massive halos. Wojtak \& Mamon (2013) performed a more refined dynamical analysis of the motions of satellites of SDSS galaxies: they found that the DM concentrations of red galaxies are consistent with the shallow, or perhaps steeper concentration-mass relation found in $\Lambda \mathrm{CDM}$ simulations. They also found that the motions of satellites around red galaxies are radial in both the outer and inner regions (while the particles in $\Lambda \mathrm{CDM}$ halos have similar radial orbits in the outer regions but have isotropic orbits in the inner regions, e.g. Lemze et al. 2013).

In analogy with spirals, it is expected that all early-type galaxies also contain a significant amount of DM, and the analyses of early-types usually relied on Newtonian dynamics. However, the MOdified Newtonian Dynamics (MOND; Milgrom 1983) theory that was well tested on spirals proved to be capable of successfully fitting early-type galaxies as well. Its basic assumption is that the acceleration due to the gravitational force does not depend simply upon the mass $m$ as in the Newtonian approach, but has a more complex form, $m / \mu\left(a / a_{0}\right)$, where $a_{0}$ is a universal constant, taken to be $a_{0}=1.35_{-0.42}^{+0.28} \times$ $10^{-8} \mathrm{~cm} \mathrm{~s}^{-2}$ (Famaey et al. 2007a). In the MOND theory, the interpolating function $\mu(x)$ is used by assuming several forms. In Sect. 3.2, the details on three interpolating functions used in this paper are given: for $a \gg a_{0}$, the Newtonian acceleration is restored $(\mu=1)$, whereas the interpolation function for $a \ll a_{0}$ becomes $\mu=a / a_{0}$.

In a recent review, Famaey \& McGaugh (2012) addressed the studies of pressure-supported stellar systems (such as early-type galaxies) in MOND. They noted that "the successes of MOND are in general a bit less impressive than in rotationally-supported ones"; as we show below, this indeed appears to be the case for the early-type galaxies that we analyze in the present paper. Milgrom (2012) used the "simple" MOND formula (see below) to analyze two isolated elliptical galaxies, NGC 720 and NGC 1521, and found that the predictions of MOND are correct but also warned about the problems of hydrostatic equilibrium and possible departures from it as a possible source of measured discrepancies.

Although our main interest is the existence and amount of $\mathrm{DM}$ in the external parts of early-type galaxies (i.e., beyond $\sim 2 R_{\mathrm{e}}$ ), we mention here that high quality literature exists on $\mathrm{DM}$ in their internal parts (interior to $\sim 1-2 R_{\mathrm{e}}$ ) based on various techniques and approaches. For example, the study of DM in early-type galaxies interior to $\sim 1 R_{\mathrm{e}}$ done by the ATLAS ${ }^{3 \mathrm{D}}$ project that used integral-field stellar kinematics suggests that the stellar mass is dominant in this region: the median fraction of DM is $13 \%$ in the sample of 250 objects (Cappellari et al. 2013a).

Our investigation of a sample of early-type galaxies is based on the application of the Jeans equation (Duncan \& Wheeler 1980), which proved to be useful in the dynamical study of both spiral galaxies (for example, the Milky Way (MW), Gnedin et al. 2010; Samurović \& Lalović 2011) and early-type galaxies (e.g. Romanowsky et al. 2003; Samurović \& Danziger 2005, 2006). The Jeans equation can be used to analyze both Newtonian (mass-follows-light and stars + NFW DM models, denoted Newtonian-noDM and Newtonian-DM models, respectively, below) and MOND models that take into account orbital anisotropies. When a declining trend of the velocity dispersion in a given galaxy is detected, it is often reasonable to interpret this as a lack of DM and the existence of a constant mass-to-light ratio. If the calculated mass-to-light ratio agrees with the value obtained using stellar population synthesis (SPS) models, then one may exclude the existence of DM (or the need to modify Newtonian dynamics).

The Jeans analysis has been used very often to study earlytype galaxies: Tiret et al. (2007) modeled NGC 3379 (already modeled by Romanowsky et al. 2003 using the Newtonian approach to claim little DM in it) and found that MOND can reproduce the observations on all scales; Samurovic (2010) showed that DM is not required out to $\sim 6.4 R_{\mathrm{e}}$ for NGC 5128 and that both Newtonian mass-follows-light and MOND models provide good fits to the line-of-sight (LOS) that are observed velocity dispersion (see also the aforementioned work of Łokas 2008). On the other hand, Salinas et al. (2012) found that MOND predictions overestimate the LOS observed velocity dispersion (which shows a declining profile) for the field elliptical NGC 7507. Schuberth et al. (2012) used their observations of a sample of 460 GCs of the early-type galaxy NGC 4636 and found that its dynamics is consistent with MOND. Richtler et al. (2008) found that the central galaxy of the Fornax cluster, NGC 1399, which shows an approximately flat velocity dispersion profile (with a high value, $\sigma \approx 300 \mathrm{~km} \mathrm{~s}^{-1}$ ) requires an additional dark halo, even with MOND (see also Samurović \& Danziger 2006). Both aforementioned galaxies, NGC 3379 and NGC 5128, have a velocity dispersion profile with a declining trend that starts at $\sim 200 \mathrm{~km} \mathrm{~s}^{-1}$ for NGC 3379 and $\sim 150 \mathrm{~km} \mathrm{~s}^{-1}$ for NGC 5128.

These works do not provide a global view of the DM content of early-type galaxies probed to distant radii with PNe or GCs. In the present study, we analyze a sample of ten massive early-type galaxies. We consider both Newtonian (mass-follows-light or 
Table 1. Kinematics data for the galaxies for the sample of GCs (see text for details).

\begin{tabular}{|c|c|c|c|c|c|c|c|c|}
\hline $\begin{array}{c}\text { Name } \\
(1) \\
\end{array}$ & $\begin{array}{c}\langle r\rangle \\
(\operatorname{arcsec}) \\
(2)\end{array}$ & $\begin{array}{r}\sigma \\
\left(\mathrm{km} \mathrm{s}^{-1}\right) \\
(3)\end{array}$ & $\begin{array}{r}\text { err_o } \\
\left(\mathrm{km} \mathrm{s}^{-1}\right) \\
(4)\end{array}$ & (5) & err_s $s_{3}$ & (7) & err_s $s_{4}$ & (9) \\
\hline \multicolumn{9}{|l|}{ NGC 1400} \\
\hline & 40 & 136 & 20 & 0.192 & 0.511 & -0.786 & 1.022 & 23 \\
\hline & 120 & 138 & 20 & 0.382 & 0.511 & -0.217 & 1.022 & 23 \\
\hline & 375 & 123 & 18 & 0.398 & 0.511 & -0.212 & 1.022 & 23 \\
\hline \multicolumn{9}{|l|}{ NGC 1407} \\
\hline & 50 & 226 & 24 & -0.093 & 0.361 & -0.650 & 0.722 & 46 \\
\hline & 125 & 251 & 25 & -0.043 & 0.350 & -0.141 & 0.700 & 49 \\
\hline & 190 & 184 & 17 & -0.199 & 0.328 & -0.618 & 0.654 & 56 \\
\hline & 265 & 217 & 21 & -0.099 & 0.330 & -0.365 & 0.660 & 55 \\
\hline & 350 & 256 & 26 & 0.454 & 0.353 & 0.781 & 0.708 & 48 \\
\hline & 500 & 224 & 21 & 0.091 & 0.324 & 0.075 & 0.648 & 57 \\
\hline & 770 & 256 & 28 & -0.273 & 0.373 & -0.236 & 0.748 & 43 \\
\hline \multicolumn{9}{|l|}{ NGC 2768} \\
\hline & 60 & 195 & 24 & 0.188 & 0.433 & -0.206 & 0.866 & 32 \\
\hline & 210 & 176 & 23 & 0.053 & 0.455 & -1.345 & 0.910 & 29 \\
\hline & 450 & 126 & 16 & -0.820 & 0.447 & 0.674 & 0.894 & 30 \\
\hline & 850 & 132 & 25 & 0.290 & 0.654 & -0.527 & 1.310 & 14 \\
\hline \multicolumn{9}{|l|}{ NGC 3115} \\
\hline & 50 & 174 & 20 & 0.181 & 0.403 & -1.021 & 0.806 & 37 \\
\hline & 150 & 198 & 19 & 0.124 & 0.328 & -0.768 & 0.654 & 56 \\
\hline & 245 & 117 & 14 & 0.001 & 0.420 & -0.629 & 0.840 & 34 \\
\hline & 413 & 139 & 21 & 0.235 & 0.511 & 0.478 & 1.022 & 23 \\
\hline \multicolumn{9}{|l|}{ NGC 3377} \\
\hline & 35 & 151 & 19 & -1.981 & 0.440 & 5.807 & 0.880 & 31 \\
\hline & 100 & 98 & 12 & -0.473 & 0.440 & 0.111 & 0.880 & 31 \\
\hline & 185 & 88 & 11 & -0.055 & 0.447 & -0.919 & 0.894 & 30 \\
\hline & 402 & 93 & 12 & 0.330 & 0.447 & -0.884 & 0.894 & 30 \\
\hline
\end{tabular}

Notes. Column (1): name of the galaxy. Column (2): central point for a given bin. Column (3): velocity dispersion for the sample of GCs in a given bin. Column (4): formal errors for the velocity dispersion of the sample of GCs in a given bin. Column (5): $s_{3}$ parameter for the sample of GCs in a given bin. Column (6): formal errors for the $s_{3}$ parameter for the sample of GCs in a given bin. Column (7): $s_{4}$ parameter for the sample of GCs in a given bin. Column (8): formal errors for the $s_{4}$ parameter for the sample of GCs in a given bin. Column (9): number of GCs in a given bin.

with additional NFW DM) and MOND dynamics (without DM, denoted MOND-noDM models hereafter), assuming isotropic, slightly tangential, or increasingly radial orbits. The best-fit mass-to-light ratios are then compared with the results of various SPS models to assess the importance of DM at a given radius of a given galaxy in the sample.

The plan of the paper is as follows: in Sect. 2, we present the observational data related to the sample of galaxies; in Sect. 3, we solve the Jeans equation for each galaxy in the sample in both the Newtonian (taking into account mass-follows-light and stars + DM models) and the MOND (taking three different models into account) approach. In Sect. 4, we analyze the models of each galaxy; in Sect. 5, we discuss our findings. Finally, we present our conclusions in Sect. 6.

\section{Observational data: Kinematics and surface density profiles}

The sample of early-type galaxies comes from the SAGES Legacy Unifying Globulars and Galaxies Survey (SLUGGS) database $^{1}$ (Pota et al. 2013, hereafter P13). SLUGGS combines Subaru/Supreme-Cam wide-field imaging with spectra obtained using the Keck/DEep Imaging Multi Object Spectrograph (DEIMOS). The sample uses high-velocity resolution data and includes galaxies with a wide range of luminosities, morphological types (within the early-type class), and those from different

\footnotetext{
1 http://sluggs.swin.edu.au
}

environments (from field galaxies to members of groups and clusters).

From P13, we decided to include ten out of twelve galaxies in our sample: we analyzed the massive early-type galaxies with the central velocity dispersion $\sigma_{0} \geq 120 \mathrm{~km} \mathrm{~s}^{-1}$. We excluded NGC 821 for which a small number of GCs was observed (58 that provide the kinematics of only two bins and the galaxy NGC 7457 because of its low central velocity dispersion $\left.\left(\sim 70 \mathrm{~km} \mathrm{~s}^{-1}\right)\right)$. Whereas, for their analysis, P13 split the GCs of each galaxy into a blue and red population, we chose to work with a total sample of GCs for each galaxy to have more clusters per bin because our goal is to determine as accurately as possible the velocity dispersion and departures from the Gaussian in the distribution of $\mathrm{GCs}^{2}$. The basic information of each galaxy is given in P13, and we only extract the most important observational data necessary for our discussion below. Throughout the paper, we assume the dimensionless Hubble constant $h_{0}=0.70$.

The kinematics of the ten galaxies from the sample based on the GCs are presented in Tables 1 and 2 and also in Figs. 1-5. The LOS velocities of GCs taken from SLUGGS were used to determine the kinematics applying standard definitions of the following quantities: the velocity dispersion $(\sigma)$, and the

2 One should note that red and blue GCs may have different kinematics: their velocity anisotropies may hence be different. Thus, they should be studied separately. However, the decision was made to study them together because their total number for the majority of galaxies is still small. 
Table 2. Kinematics data for the galaxies for the sample of GCs (see text for details).

\begin{tabular}{|c|c|c|c|c|c|c|c|c|}
\hline Name & $\begin{array}{c}\langle r\rangle \\
(\operatorname{arcsec}) \\
(2)\end{array}$ & $\begin{array}{c}\sigma \\
\left(\mathrm{km} \mathrm{s}^{-1}\right) \\
(3)\end{array}$ & $\begin{array}{r}\text { Rrr_o } \\
\left(\mathrm{km} \mathrm{s}^{-1}\right) \\
(4)\end{array}$ & (5) & Rrr $\_s_{3}$ & (7) & Rrr__s $s_{4}$ & $N$ \\
\hline \multicolumn{9}{|l|}{ NGC 4278} \\
\hline & $\begin{array}{r}45 \\
120\end{array}$ & $\begin{array}{l}225 \\
171\end{array}$ & $\begin{array}{l}21 \\
16\end{array}$ & $\begin{array}{l}0.033 \\
0.130\end{array}$ & $\begin{array}{l}0.324 \\
0.330\end{array}$ & $\begin{array}{r}0.236 \\
-0.160\end{array}$ & $\begin{array}{l}0.648 \\
0.660\end{array}$ & $\begin{array}{l}57 \\
55\end{array}$ \\
\hline & 180 & 197 & 20 & -0.090 & 0.343 & 0.002 & 0.686 & 51 \\
\hline & 255 & 141 & 15 & -0.232 & 0.369 & -0.480 & 0.738 & 44 \\
\hline & 400 & 154 & 16 & 0.106 & 0.350 & -0.636 & 0.700 & 49 \\
\hline \multicolumn{9}{|l|}{ NGC 4365} \\
\hline & 35 & 279 & 29 & -0.164 & 0.353 & -0.398 & 0.708 & 48 \\
\hline & 110 & 251 & 23 & -0.054 & 0.316 & -0.448 & 0.632 & 60 \\
\hline & 175 & 221 & 24 & -0.310 & 0.382 & -0.376 & 0.766 & 41 \\
\hline & 250 & 241 & 25 & -0.015 & 0.365 & -0.234 & 0.730 & 45 \\
\hline & 506 & 246 & 23 & -0.432 & 0.324 & 0.816 & 0.648 & 57 \\
\hline \multicolumn{9}{|l|}{ NGC 4486} \\
\hline & 50 & 356 & 32 & -1.130 & 0.306 & 2.072 & 0.612 & 64 \\
\hline & 110 & 316 & 33 & -0.447 & 0.361 & -0.435 & 0.722 & 46 \\
\hline & 135 & 359 & 35 & 0.157 & 0.339 & 0.396 & 0.680 & 52 \\
\hline & 170 & 364 & 34 & 0.307 & 0.328 & 0.197 & 0.654 & 56 \\
\hline & 205 & 330 & 30 & 0.235 & 0.319 & -0.310 & 0.638 & 59 \\
\hline & 240 & 447 & 48 & 0.921 & 0.369 & 1.405 & 0.738 & 44 \\
\hline & 280 & 388 & 40 & 0.605 & 0.361 & 0.950 & 0.722 & 46 \\
\hline & 330 & 328 & 32 & 0.061 & 0.333 & -0.932 & 0.666 & 54 \\
\hline & 400 & 503 & 50 & -0.208 & 0.346 & 0.390 & 0.692 & 50 \\
\hline & 505 & 452 & 43 & -0.334 & 0.333 & 0.255 & 0.666 & 54 \\
\hline & 660 & 323 & 34 & 0.185 & 0.361 & 0.158 & 0.722 & 46 \\
\hline & 850 & 307 & 33 & -0.113 & 0.369 & -0.132 & 0.738 & 44 \\
\hline & 1075 & 323 & 34 & 0.185 & 0.361 & 0.158 & 0.722 & 46 \\
\hline & 1400 & 276 & 28 & -0.393 & 0.357 & 2.538 & 0.714 & 47 \\
\hline & 6275 & 334 & 45 & 0.429 & 0.463 & -0.193 & 0.926 & 28 \\
\hline \multicolumn{9}{|l|}{ NGC 4494} \\
\hline & 50 & 99 & 10 & 0.018 & 0.050 & 0.264 & 0.035 & 50 \\
\hline & 150 & 99 & 11 & 0.046 & 0.057 & 0.328 & 0.040 & 38 \\
\hline & 343 & 102 & 14 & -0.028 & 0.069 & 0.497 & 0.048 & 26 \\
\hline \multicolumn{9}{|l|}{ NGC 5846} \\
\hline & 70 & 219 & 23 & -0.056 & 0.365 & -0.163 & 0.730 & 45 \\
\hline & 195 & 287 & 28 & 0.153 & 0.337 & 0.706 & 0.672 & 53 \\
\hline & 300 & 220 & 22 & -0.448 & 0.353 & -0.161 & 0.708 & 48 \\
\hline & 590 & 248 & 26 & 0.168 & 0.365 & -0.822 & 0.730 & 45 \\
\hline
\end{tabular}

Notes. The same as in Table 1 .

skewness and kurtosis parameters, $s_{3}$ and $s_{4}$, which describe asymmetric and symmetric departures from the Gaussian in each bin, respectively. The typical velocity errors for the objects are below $20 \mathrm{~km} \mathrm{~s}^{-1}$, and they rarely exceed $30 \mathrm{~km} \mathrm{~s}^{-1}$.

We calculate the velocity dispersion from the observations using the standard definition and the NAG routine G01AAF. The departures from the Gaussian are calculated using the standard formulae for unbiased estimators (Joanes \& Gill 1998) that also include the errors:

$s_{3}=\frac{N}{(N-1)(N-2)} \sum_{i=1}^{N}\left(\frac{v_{i}-v_{\text {sys }}}{\sigma}\right)^{3} \pm \sqrt{\frac{6}{N}}$,

and

$$
\begin{aligned}
s_{4}= & \frac{N(N+1)}{(N-1)(N-2)(N-3)} \sum_{i=1}^{N}\left(\frac{v_{i}-v_{\text {sys }}}{\sigma}\right)^{4} \\
& -3 \frac{(N-1)^{2}}{(N-2)(N-3)} \pm \sqrt{\frac{24}{N}}
\end{aligned}
$$

where $N$ is the number of object per bin, $v_{i}$ is the velocity of the given object, $v_{\text {sys }}$ is the systemic velocity, and $\sigma$ is the velocity dispersion. The velocity moments were computed in radial bins of roughly equal number of velocities with 40 velocities in all galaxies, except NGC 1400, NGC 2768 and NGC 3377. We considered the LOS kurtosis for a first guess at the level of velocity anisotropy: positive, null, or negative kurtosis is indicative of radial, isotropic, and tangential orbits, respectively (Merritt 1987; Gerhard 1993). However, we note that rotation also affects the kurtosis (Dekel et al. 2005), and one should be careful in the interpretation of the measured kurtosis. The values of the kurtosis parameters calculated in this paper fully agree with those presented in P13.

From Tables 1 and 2 and Figs. 1-5, one can see that the profiles of the LOS velocity dispersion beyond $\sim 1 R_{\mathrm{e}}$ (for the galaxies for which we have several observed points) are approximately constant for all the galaxies in the sample, except for NGC 2768 and NGC 3115, where the velocity dispersions drop from $\sim R_{\mathrm{e}}$ to $\sim 2 R_{\mathrm{e}}$, and are constant at larger projected radii. One can also see that the values of kurtosis, $s_{4}$, in the sample are almost always close to zero at all radii, implying quasi-isotropic 

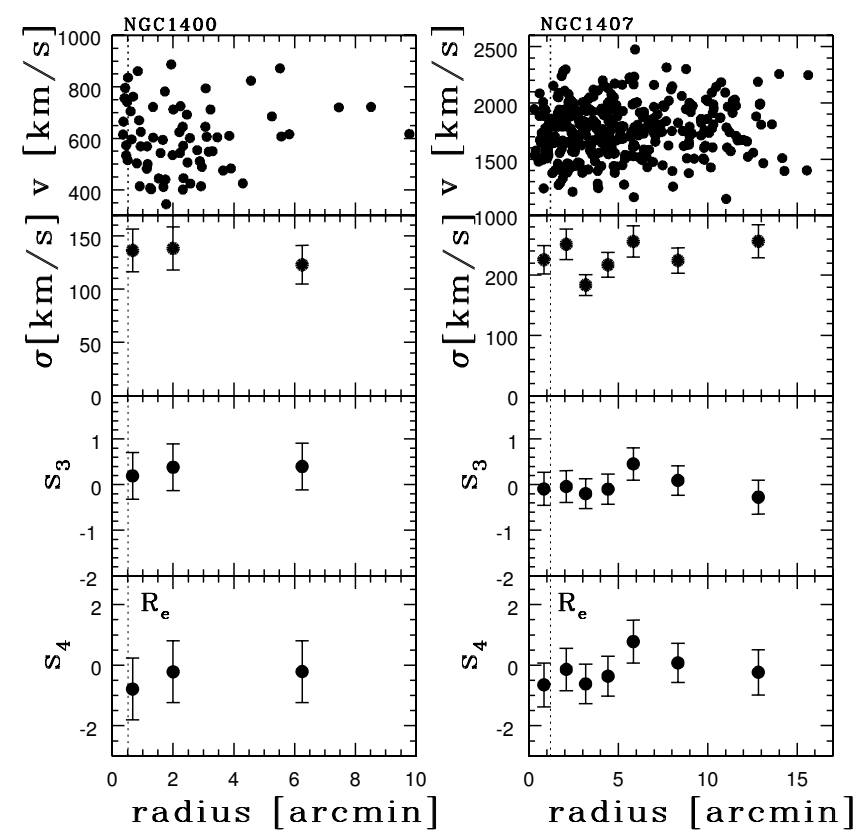

Fig. 1. Kinematics of NGC 1400 (left) and NGC 1407 (right) based on the sample of GCs from the SLUGGS database. From top to bottom: LOS velocity of the GCs in $\mathrm{km} \mathrm{s}^{-1}$; velocity dispersion in $\mathrm{km} \mathrm{s}^{-1}$ calculated in a given bin and the $s_{3}$ and $s_{4}$ parameters, which describe symmetric and asymmetric departures from the Gaussian, respectively. The vertical dotted line in both plots denotes one effective radius. See text for details.
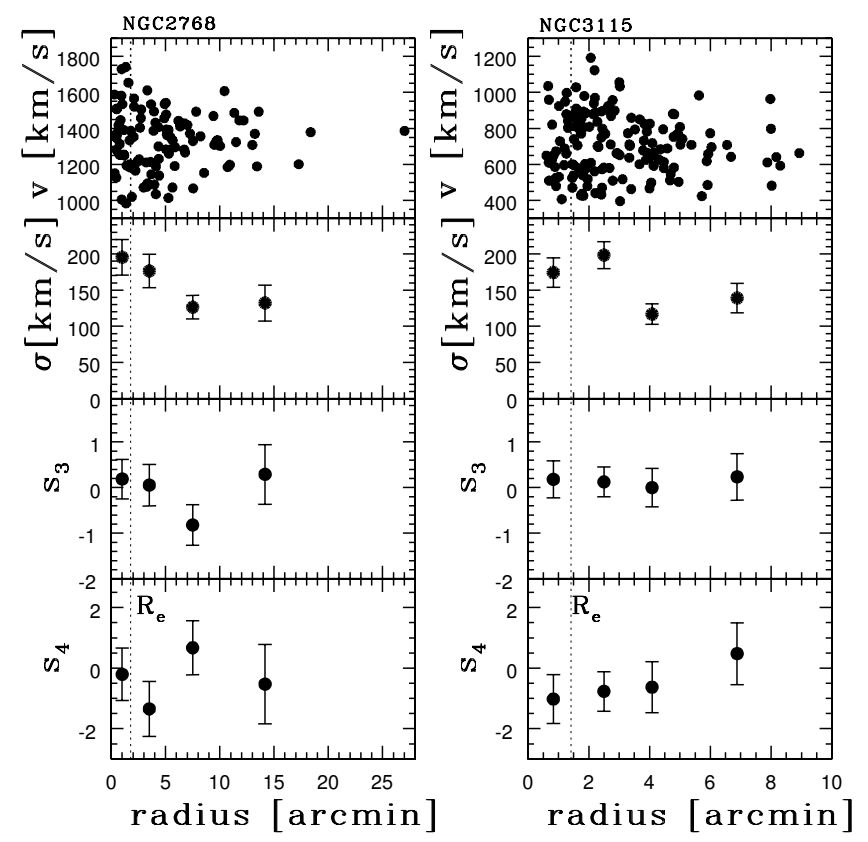

Fig. 2. Kinematics of NGC 2768 (left) and NGC 3115 (right) based on the sample of GCs from the SLUGGS database. The meaning of symbols is the same as in Fig. 1.

orbits in the absence of rotation. The exceptions are NGC 3115 , whose inner and intermediate radii have moderately negative kurtoses, and NGC 4486 (M 87), whose innermost and outermost radial bins (among 15) have positive kurtoses.

In Table 3, we present the values of the GC density-profile exponent $\gamma$ coming from the power-law fits, $\gamma=-\mathrm{d} \ln N_{\mathrm{cl}} / \mathrm{d} \ln R$ for all the galaxies in the sample. These values are used in the Jeans analysis below. From this Table, one can see that GCs in
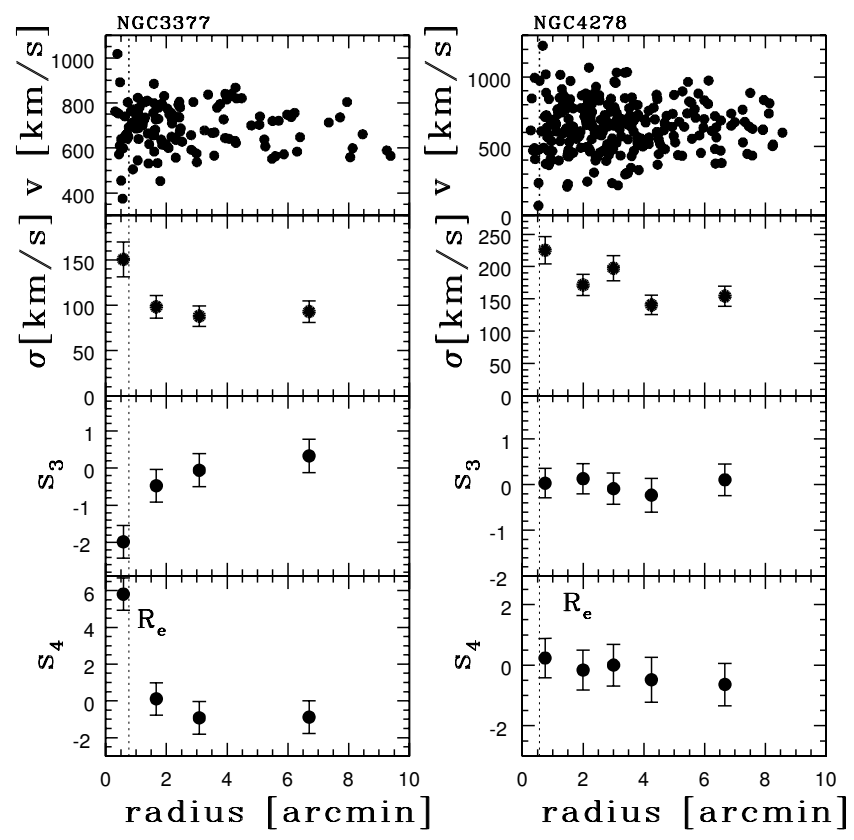

Fig. 3. Kinematics of NGC 3377 (left) and NGC 4278 (right) based on the sample of GCs from the SLUGGS database. The meaning of symbols is the same as in Fig. 1. Note the different scale of the $s_{4}$ parameter of NGC 3377.
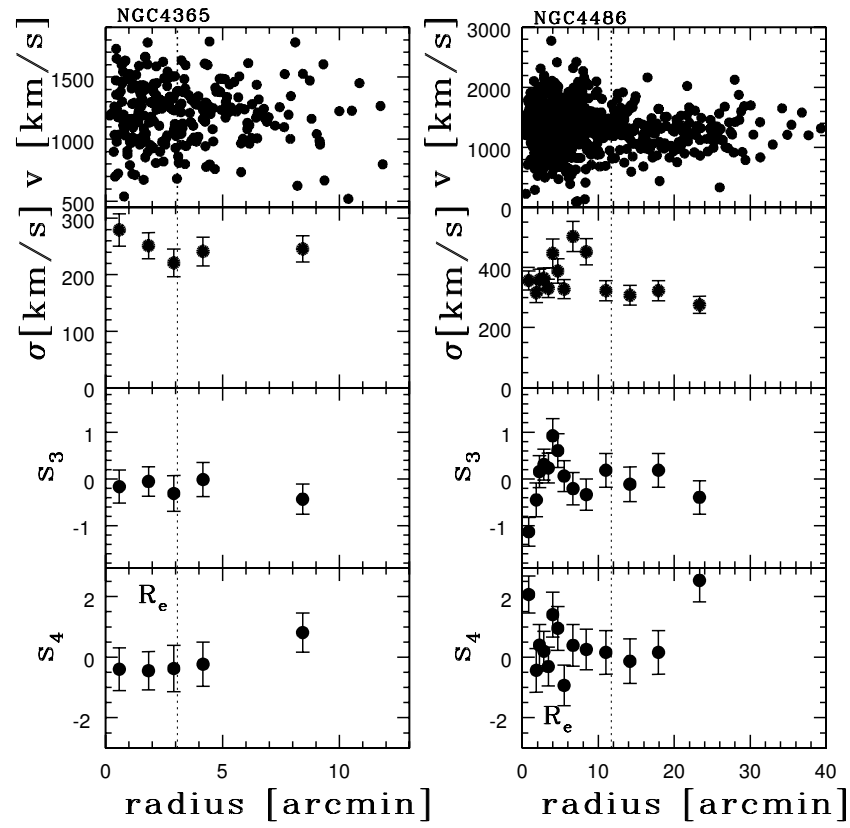

Fig. 4. Kinematics of NGC 4365 (left) and NGC 4486 (right) based on the sample of GCs from the SLUGGS database. The meaning of symbols is the same as in Fig. 1.

four galaxies can be fit using a single value of the $\gamma$ parameter; for the remaining seven galaxies the radial distribution was fit using the Sérsic model,

$\Sigma(R) \propto \exp \left[-b_{n}\left(\frac{R}{R_{\mathrm{e}}}\right)^{1 / n}\right]$,

where $b_{n}=1.9992 n-0.3271$ and $R_{\mathrm{e}}$ is the effective radius of the GC system (see e.g. Graham et al. 2003, where in each case the Sérsic index $n_{\text {SCrsic }}^{\mathrm{GC}}$ was given) and also uses two values of the $\gamma$ parameter, $\gamma_{\text {in }}$ and $\gamma_{\text {our }}$, for the inner and outer 
Table 3. GCs data and fits for the Sérsic stellar surface brightness for the galaxies in the sample and fits of the radial distribution of GCs for the galaxies in the sample.

\begin{tabular}{crrrrrrrrrr}
\hline \hline $\begin{array}{c}\text { name } \\
(1)\end{array}$ & $\begin{array}{c}R_{\max }\left[{ }^{\prime \prime}\right] \\
(2)\end{array}$ & $\begin{array}{c}R_{\max } / R_{\mathrm{e}} \\
(3)\end{array}$ & $\begin{array}{c}N_{\mathrm{GC}} \\
(4)\end{array}$ & \multicolumn{1}{c}{$\begin{array}{c}\gamma^{\mathrm{T}} \\
(6)\end{array}$} & $\begin{array}{c}\gamma_{\text {in }} \\
(7)\end{array}$ & $\begin{array}{c}\gamma_{\text {out }} \\
(8)\end{array}$ & $\begin{array}{c}n_{\text {Sersic }}^{\mathrm{GC}} \\
(9)\end{array}$ & $\begin{array}{c}\bar{\chi}_{\gamma}^{2} \text { (in) } \\
(10)\end{array}$ & $\begin{array}{c}\bar{\chi}_{\gamma}^{2} \text { (out) } \\
(11)\end{array}$ & $\begin{array}{c}\bar{\chi}_{\mathrm{s}} \\
(12)\end{array}$ \\
\hline NGC 1400 & 375 & 12.10 & 69 & $1.72 \pm 0.20$ & - & - & - & $0.40^{\mathrm{T}}$ & - & - \\
NGC 1407 & 770 & 10.69 & 354 & - & $0.94 \pm 0.34$ & $2.55 \pm 1.39$ & $1.68 \pm 0.02$ & 0.14 & 0.01 & 0.04 \\
NGC 2768 & 850 & 9.14 & 105 & $1.79 \pm 0.19$ & - & - & - & $1.09^{\mathrm{T}}$ & - & - \\
NGC 3115 & 413 & 4.86 & 150 & - & $1.34 \pm 0.84$ & $4.17 \pm 0.31$ & $1.75 \pm 0.05$ & 1.84 & 0.09 & 1.14 \\
NGC 3377 & 402 & 8.74 & 122 & - & $1.38 \pm 0.82$ & $1.93 \pm 0.43$ & $1.80 \pm 0.20$ & 1.33 & 0.04 & 4.20 \\
NGC 4278 & 400 & 12.50 & 256 & - & $0.90 \pm 1.20$ & $1.87 \pm 0.58$ & $1.90 \pm 0.15$ & 0.36 & 0.18 & 0.35 \\
NGC 4365 & 506 & 2.75 & 251 & - & $0.84 \pm 0.20$ & $2.10 \pm 0.66$ & $1.86 \pm 0.15$ & 0.34 & 0.18 & 0.44 \\
NGC 4486 & 1400 & 1.98 & 708 & $0.68 \pm 0.04$ & - & - & - & $5.33^{\mathrm{T}}$ & - & - \\
NGC 4494 & 343 & 6.47 & 114 & - & $0.73 \pm 0.93$ & $2.18 \pm 0.87$ & $1.62 \pm 0.10$ & 0.90 & 0.15 & 1.24 \\
NGC 5846 & 590 & 9.67 & 191 & - & $0.81 \pm 0.42$ & $4.15 \pm 0.46$ & $1.42 \pm 0.10$ & 0.45 & 0.12 & 1.06 \\
\hline
\end{tabular}

Notes. Outermost radial bin for each galaxy in the sample, $R_{\max }$, is expressed in arcsecs and in units of $R / R_{\mathrm{e}}$ for each object. The surface density of the given galaxy can be fit with a single value of the $\gamma$ parameter in three cases (NGC 1400, NGC 2768, and NGC 4486). For other galaxies in the sample, the fits were obtained in the inner regions (given by $\gamma_{\text {in }}$ ) and in the outer regions (given by $\gamma_{\text {out }}$ ); for these galaxies, the Sérsic index $\left(n_{\text {Sersic }}^{\mathrm{GC}}\right.$ ), which provides a fit throughout the whole galaxy, is given in the text below for each galaxy in the sample. For each case, the reduced $\bar{\chi}^{2}$ values are given. See text for details.
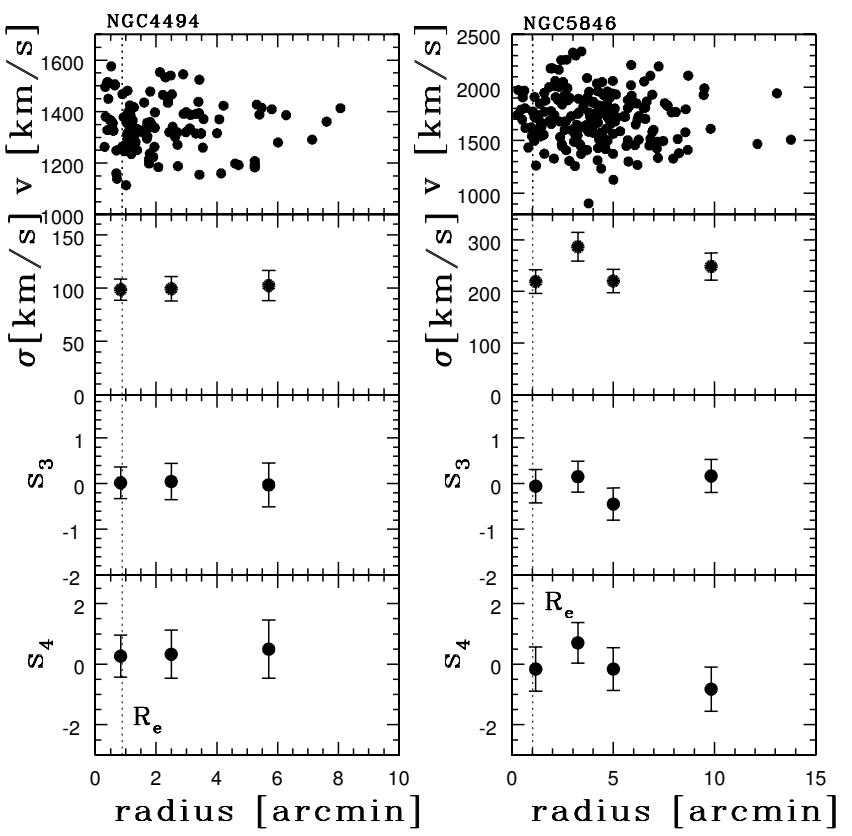

(see Carlberg et al. 1997; Katgert et al. 2004). We are interested in the problem of DM, which influences outer regions of the galaxies, and thus for several galaxies where significant departures from the power law were detected, we separately determined the surface density profile for their outer part (see Figs. 6 and 7). The change of the slope in $\gamma$ of \pm 0.50 would result in the change of the established mass-to-light ratio of approximately $\pm 25 \%$. Only more detailed observations of the galaxies in the sample will provide a more complete picture, and at the moment, one should bear this caveat in mind.

\section{Dynamical models}

For all the early-type galaxies in our sample, we solve the Jeans equation (e.g. Binney \& Tremaine (2008)) in a spherical approximation for both Newtonian and MOND approaches:

$\frac{\mathrm{d} \sigma_{r}^{2}}{\mathrm{~d} r}+(\alpha+2 \beta) \frac{\sigma_{r}^{2}}{r}=a_{\mathrm{N} ; \mathrm{M}}+\frac{v_{\mathrm{rot}}^{2}}{r}$,

where $a_{\mathrm{N} ; \mathrm{M}}$ is an acceleration term, which is different for each approach. In the Newtonian ("N") approach, it is equal to $a_{\mathrm{N}}=$

Fig. 5. Kinematics of NGC 4494 (left) and NGC 5846 (right) based on the sample of GCs from the SLUGGS database. The meaning of symbols is the same as in Fig. 1.

parts, respectively. The fits for all the galaxies were obtained using the spectroscopic data from the SLUGGS database and are shown in Figs. 6 and 7 . For the fitting, the simple $\chi^{2}$ procedures were adopted based on the ordinary least squares and the Levenberg-Marquardt algorithm. Both Sérsic index and effective radius were fit in the given ranges. For linear fits, when one value of the $\gamma$-parameters is used, all observational points are used. When two regions exist (inner and outer parts), two separate groups of points were used without intersection. The GC surface density profiles obtained here are in agreement with those obtained in P13.

The problem of radial incompleteness in the inner regions of the galaxies in the sample may occur because of the high surface brightness of the host galaxies. The estimates of the deprojected tracer density are also affected, and through the Jeans equation, the resulting mass profiles are affected $-G M(r) / r^{2}$, and for MOND ("M"), $a_{\mathrm{M}}$ satisfies (Milgrom 1983)

$a_{\mathrm{M}} \mu\left(\frac{a_{\mathrm{M}}}{a_{0}}\right)=a_{\mathrm{N}}$,

which is used in Sect. 3.2 on MOND. In Eq. (4), $\sigma_{r}$ is the radial stellar velocity dispersion, $\alpha=\mathrm{d} \ln v / \mathrm{d} \ln r$ is the slope of tracer density $v$. The exponents of the power-law fits of the surface density for each galaxy in the sample are given in Table 3: for example, the radial distribution for NGC 1400 was fit with the exponent equal to -1.72 , which means that $\alpha$ used in the Jeans equation is $\alpha=-2.72$. The rotation speed $v_{\text {rot }}$ for each galaxy is given below in the description of each galaxy and is neglected in the Jeans equation when it is consistent with zero. The values of the rotation speed are taken from P13 (their Table 6), determined using GCs from the SLUGGS sample and listed in Table 4. In Table 4, each object in the sample was designated with " $\mathrm{f}$ " (fast rotator) or " $\mathrm{s}$ " (slow rotator): the information is taken from Arnold et al. (2014, their Table 1), who used the original criteria from Emsellem et al. (2007) to calculate (for galaxies from the SLUGGS sample missing in the original work 
Table 4. Basic observational data of the galaxies in the sample.

\begin{tabular}{|c|c|c|c|c|c|c|c|c|c|c|c|}
\hline Name & $\begin{array}{c}\text { Distance } \\
{[\mathrm{Mpc}]} \\
(2)\end{array}$ & $\begin{array}{c}1 \text { arcsec } \\
{[\mathrm{pc}]} \\
(3)\end{array}$ & $\begin{array}{c}1 \text { arcmin } \\
{[\mathrm{kpc}]} \\
(4)\end{array}$ & $M_{B}$ & Morph. type & Environ. & $\begin{array}{c}R_{\mathrm{e}}^{*} \\
{[\operatorname{arcsec}]} \\
(8) \\
\end{array}$ & $\begin{array}{c}v_{\text {rot }} \\
{\left[\mathrm{km} \mathrm{s}^{-1}\right]} \\
(9)\end{array}$ & Isophotes & Profile & Rotator \\
\hline NGC 1400 & 26.8 & 129.98 & 7.80 & -20.35 & S0/E0 & G & 31 & $10 \pm 23$ & $\cap^{1}$ & $0^{2}$ & $\mathrm{f}$ \\
\hline NGC 1407 & 26.8 & 129.98 & 7.80 & -21.49 & E0 & G & 72 & $39 \pm 18$ & $\cap^{2}$ & $0^{2}$ & $\mathrm{~s}$ \\
\hline NGC 2768 & 21.8 & 105.73 & 6.34 & -21.26 & $\mathrm{E} 6 / \mathrm{SO}_{1 / 2}$ & G & 93 & $57 \pm 19$ & $?^{3}$ & $\mathrm{D}^{5}$ & f \\
\hline NGC 3115 & 9.4 & 45.59 & 2.74 & -19.94 & SO & $\mathrm{F}$ & 85 & $100 \pm 14$ & $\backslash^{1}$ & $\mathrm{D}^{6}$ & f \\
\hline NGC 3377 & 10.9 & 52.87 & 3.18 & -19.32 & E6 & G & 46 & $18 \pm 13$ & $\lambda^{1}$ & $\mathrm{D}^{5}$ & f \\
\hline NGC 4278 & 15.6 & 75.66 & 4.54 & -19.50 & E1-2 & G & 34 & $28 \pm 13$ & $\cap^{1}$ & $\mathrm{~B}^{5}$ & f \\
\hline NGC 4365 & 23.3 & 113.00 & 6.78 & -21.00 & E3 & G & 184 & $26 \pm 24$ & $\cap^{1}$ & $\mathrm{~B}^{7}$ & s \\
\hline NGC 4486 & 17.2 & 83.42 & 5.01 & -22.05 & E0 & $\mathrm{C}$ & 704 & $25 \pm 18$ & $\cap^{1}$ & $\mathrm{~B}^{5}$ & \\
\hline NGC 4494 & 16.6 & 80.51 & 4.83 & -21.07 & E1-E2 & G & 53 & $62 \pm 12$ & $\backslash^{1}$ & $\mathrm{D}^{7}$ & f \\
\hline NGC 5846 & 24.2 & 117.37 & 7.04 & -21.34 & E0 & G & 61 & $5 \pm 38$ & $?^{4}$ & $\mathrm{~B}^{5}$ & $\mathrm{~s}$ \\
\hline
\end{tabular}

Notes. Column (1): name of the galaxy. Column (2): distances to the galaxies in the sample. The distances found in P13 were taken from Tonry et al. (2001), with corrections by Mei et al. (2007), see P13 for details. Column (3): 1 arcsec expressed in parsecs. Column (4): 1 arcmin expressed in kiloparsecs. Column (5): B-band absolute magnitude (taken from HyperLeda). Column (6): morphological type (taken from P13). Column (7): environment of each galaxy (taken from Arnold et al. (2014); "G" means that the galaxy belongs to a group, "F" is a field galaxy and "C" means that the galaxy belongs to a cluster). Column (8): stellar effective radius (taken from P13, except for the values of NGC 4365 and NGC 4486 that are taken from Kormendy et al. 2009). Column (9): rotational velocity (taken from P13, based on GCs, see their Table 6). Column (10): profile type (" $\cap$ " is core, "Y" is power law and "?" is unknown due to observational problems, such as obscuration). Column (11): mean isotope shape ("D" indicates disky isophotes, "B" indicates boxy isophotes and " 0 " indicates pure ellipses). The references for the values found in Cols. (10) and (11) are as follows. (1): Lauer et al. (2007), (2): Spolaor et al. (2008), (3): Lauer et al. (2005), (4): Rest et al. (2001), (5): Emsellem et al. (2007), (6): Nieto et al. (1991), (7): Bender et al. (1989). Column (12): central rotator type ("s" indicates slow and "f" indicates slow rotators); the designations come from Arnold et al. (2014), see their Table 1 and also Emsellem et al. (2007).

of Emsellem et al.) the values of the parameter $\lambda_{R}$ to quantify the specific angular momentum in the stellar component of galaxies.

When calculating the Jeans equation, we include only the stellar component in our analysis: we neglect the hot gas observed in some galaxies (such as the central galaxy of the Virgo cluster, NGC 4486) because it is known that the total mass of hot gas in massive early-type galaxies varies up to at least several $10^{10} M_{\odot}$ (about $1 \%$ ) of the total stellar mass (Mathews \& Brighenti 2003).

The non-spherical nature of the GC dispersion is expressed through the following well-known equation:

$\beta=1-\frac{\overline{v_{\theta}^{2}}}{\sigma_{r}^{2}}$,

where $\overline{v_{\theta}^{2}}={\overline{v_{\theta}}}^{2}+\sigma_{\theta}^{2}$ and $0<\beta<1$ mean that the orbits are predominantly radial (equivalent to $s_{4}>0$ ), whereas the orbits for $-\infty \leq \beta<0$ are mostly tangential (equivalent to $s_{4}<0$ ) (Gerhard 1993).

Our Jeans analysis is based on the observed LOS velocity dispersion. In the literature, one can find other approaches to lift the mass/anisotropy degeneracy, for example, by fitting the LOS velocity kurtosis profiles together with the LOS velocity dispersion profiles (Łokas 2003; Łokas \& Mamon 2003; Richardson \& Fairbairn 2013).

In this paper, following P13, the galactocentric distances in each galaxy are expressed as the equivalent radius:

$R=\sqrt{q X^{2}+\frac{Y^{2}}{q}}$,

where $q$ is the axis ratio (ratio of minor over major axis) of the given galaxy and $X$ and $Y$ are Cartesian coordinates of the given object in the rest frame of the galaxy. The center of the galaxy coincides with the origin of the coordinate system, and $X(Y)$ is aligned with the photometric major (minor) axis. The values of the $q$ parameter are taken from P13; we stress here that this is the stellar $q$ parameter, and we implicitly assumed that the GC systems have the same orientations and flattenings as the stars. We fold the rotation and the dispersion profile into a root mean square (rms) velocity profile $v_{\text {rms }}=\sqrt{v_{\text {rot }}^{2}+\sigma^{2}}$, where $v_{\text {rot }}$ is the rotation indicated for each galaxy in the sample and $\sigma$ is the dispersion. The rotation is always taken into account in our models when it is inconsistent with the zero value, and the values of $v_{\text {rot }}$ come from P13. Although most of the galaxies in the sample are flattened in their inner stellar regions, they become more spherical and thus we use spherical models in their outer parts. Furthermore, the comparison of the results are made with D12, who, for the sake of simplicity, also used spherical models.

Naturally, more sophisticated (and also computationally more demanding) approaches are possible, such as Schwarzschild's (1979) orbit superposition modeling or the NMAGIC modeling (de Lorenzi et al. 2009), but as D12 warn "it is not obvious whether this extra complication makes any appreciable difference to the mass estimates".

In this paper, we study three cases of anisotropies:

- the isotropic case $(\beta=0)$;

- the mildly tangential case for which there is a hint from the observed kinematics $(\beta=-0.20)$. The negative values of the $s_{4}$ parameter calculated for all galaxies in the sample suggest that there is a possibility of the existence of tangential anisotropies, and thus, $\beta=-0.20$ was used, reducing the adopted value of $\beta=-0.20$ would lead to smaller a massto-light ratio. The fits obtained using this anisotropy are presented in Tables 6 and 7 with another two cases of anisotropy and are not shown in figures because the curves based on this anisotropy are very close to those based on isotropic cases, albeit characterized by smaller mass-to-light ratios;

- the theoretically based value $\left(\beta(r)=\beta_{\infty} r /\left(r+r_{a}\right)\right)$, where $\beta_{\infty} \simeq 0.5$ and $r_{a} \simeq 1.4 R_{\mathrm{e}}$. This estimate (radially dominated) comes from theoretical expectations from merging 


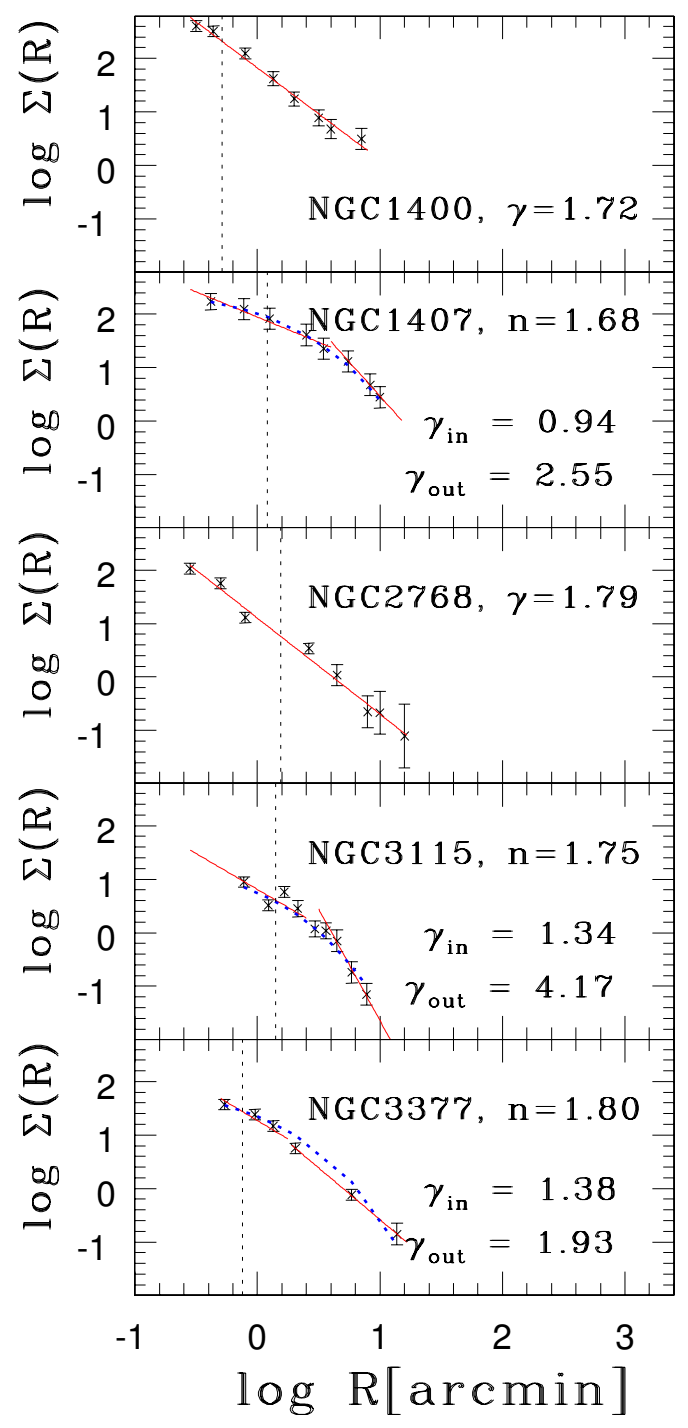

Fig. 6. Surface density profiles of GCs of five of the galaxies from the sample obtained using spectroscopic data. A power law is fit with the solid red lines to the radial surface density of GCs: $N_{\mathrm{cl}} \propto R^{-\gamma}$ for two galaxies (NGC 1400 and NGC 2768). For the remaining three galaxies, the Sérsic fit, determined by the parameter $n$ is also presented using the dotted blue lines: in such cases, a power law is fit in the inner and outer regions separately with the solid red lines (see text for details).

collisionless systems (Mamon \& Łokas 2005). The values of the calculated $\beta$ parameter are denoted $\beta_{\text {lit }}$ in tables and plots below.

The projected LOS velocity dispersion (e.g. Binney \& Mamon 1982) for all the models for the galaxies in the sample is given as

$\sigma_{\mathrm{p}}^{2}(R)=\frac{\int_{R}^{r_{\mathrm{t}}} \sigma_{r}^{2}(r)\left[1-(R / r)^{2} \beta\right] \rho(r)\left(r^{2}-R^{2}\right)^{-1 / 2} r \mathrm{~d} r}{\int_{R}^{r_{\mathrm{t}}} \rho(r)\left(r^{2}-R^{2}\right)^{-1 / 2} r \mathrm{~d} r}$,

where the truncation radius, $r_{\mathrm{t}}$, extends beyond the observed kinematical point of the highest galactocentric radius. Equation (8) is effectively a double integral, because $\sigma_{r}$ inside is a single integral itself in terms of the total mass and tracer density profiles. For the simple anisotropy models, such as those used in the present paper, the single integral expression for $\sigma_{\mathrm{p}}(R)$ is provided in the Appendix of Mamon \& Łokas (2005).

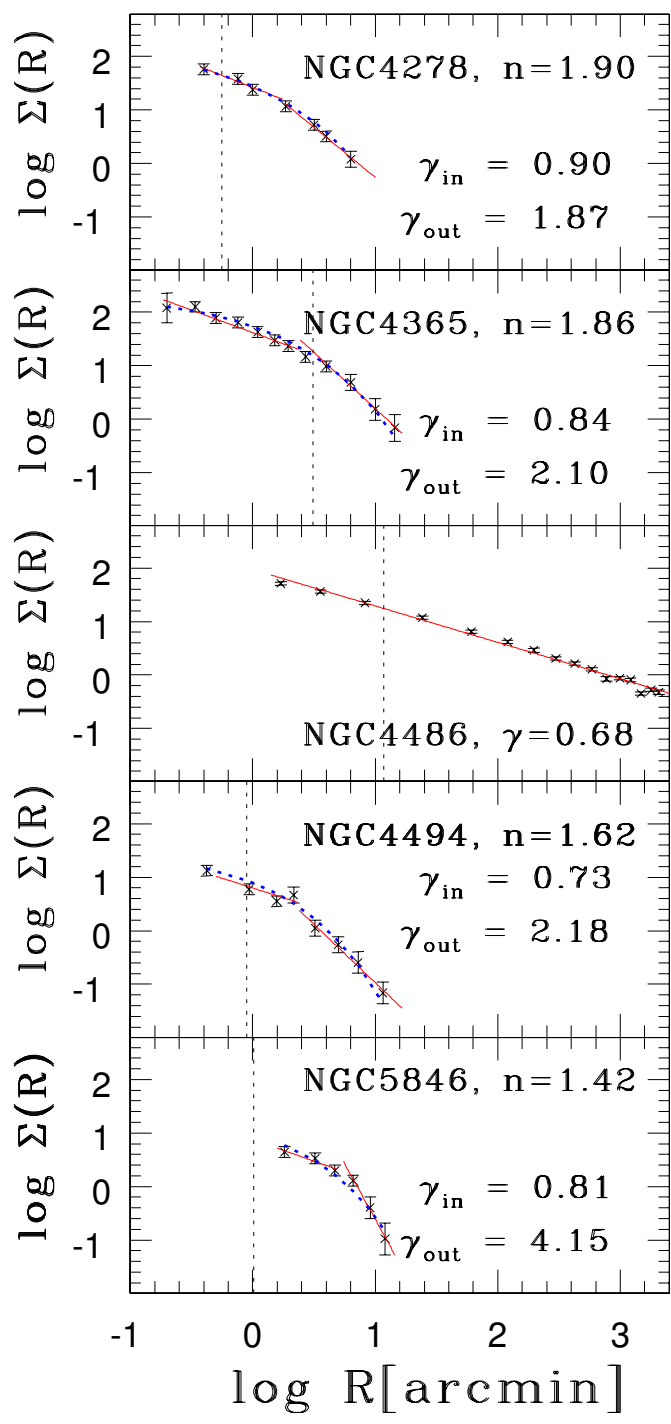

Fig. 7. Surface density profiles of GCs of the remaining five galaxies from the sample. A power law is fit with the solid red line to the radial surface density of GCs: $N_{\mathrm{cl}} \propto R^{-\gamma}$ for NGC 4486 . For the remaining four galaxies, the Sérsic fit, determined by the parameter $n$, is also presented using the dotted blue lines. In such cases, a power law is fit in the inner and outer regions separately with the solid lines (see text for details).

\subsection{Newtonian models}

For a constant mass-to-light ratio model, we used a constant mass-to-light ratio $\left(M / L_{*}\right)$ Sérsic model (see Romanowsky et al. 2009) that uses a galaxy's field stars and for which the circular velocity is

$$
\begin{aligned}
v_{\mathrm{c}}^{2}(r) & \equiv \frac{G M(r)}{r} \\
& =\frac{G\left(M / L_{*}\right) L_{*}}{r}\left\{1-\frac{\Gamma\left[(3-p) n_{*},\left(r / a_{\mathrm{s}}^{*}\right)^{1 / n_{*}}\right]}{\Gamma\left[(3-p) n_{*}\right]}\right\},
\end{aligned}
$$

where $n_{*}$ and $a_{\mathrm{s}}^{*}$ are the Sérsic index and scale radius for the stellar component, and $p$ is a function of $n_{*}$. Mamon \& Łokas (2005) provide the details regarding the Sérsic optical surface brightness that is deprojected: using the work of Lima Neto et al. (1999; see also Prugniel \& Simien 1997, for the derivation of the original formula), it can be shown that $p \simeq 1.0-0.6097 / n_{*}+$ $0.05463 / n_{*}^{2}$ and for $b \simeq 2 n_{*}-1 / 3+0.009876 / n_{*}$, it follows 
$a_{\mathrm{s}}^{*} \simeq\left\{b\left[n_{*}^{(\mathrm{fit})}\right]\right\}^{-n_{*}^{(\mathrm{fit})}} R_{\mathrm{e}}^{(\mathrm{fit})}$, where "fit" denotes the fit value of the given quantity. Parameter $a_{\mathrm{s}}^{*}$ was used only in solving Eq. (9).

For our Newtonian model with DM, we add to the stellar component an NFW (Navarro et al. 1997) DM halo. The NFW density has the following form:

$\rho(x)=\frac{4 \rho_{\mathrm{s}}}{x(x+1)^{2}}$,

and the enclosed mass becomes:

$M(x)=4 \pi \rho_{\mathrm{s}} r_{\mathrm{s}}^{3}\left[\ln (1+x)-\frac{x}{1+x}\right]$,

where $x=r / r_{\mathrm{s}}, r_{\mathrm{s}}$ is the scale radius at which the logarithmic slope of the NFW density profile is -2 , while $\rho_{\mathrm{s}}$ is the density at that radius. This halo is added to the stellar mass calculated using the Sérsic model as given above.

The free parameters of the NFW model are thus: $M / L_{*}$, $\rho_{\mathrm{s}}$ and $r_{\mathrm{s}}$. As usual (see e.g. Napolitano et al. 2009, 2011), one can parametrize the halo using the virial mass $\left(M_{\mathrm{vir}} \equiv\right.$ $\left.4 \pi \Delta_{\text {vir }} \rho_{\text {crit }} r_{\text {vir }}^{3} / 3\right)$ and concentration $\left(c_{\text {vir }} \equiv r_{\text {vir }} / r_{\mathrm{s}}\right)$, where the critical density is $\rho_{\text {crit }}=1.37 \times 10^{-7} M_{\odot} \mathrm{pc}^{-3}$ and the critical overdensity is $\Delta_{\text {vir }}=101$. The estimated values of the concentration parameter are used below when we compare the results obtained using the SLUGGS sample with theoretical expectations. In the estimates of the virial mass and the concentration index, the uncertainties related to anisotropies were taken into account, so the listed error bars in Table 8 (see below) cover the uncertainties due to all three tested Jeans models (isotropic, radially dominated, and tangentially dominated).

\subsection{MOND models}

We tested several MOND models using the Jeans equation in the spherical approximation.

The MOND acceleration, $a_{\mathrm{M}}$, is given by Eq. (5). The following three MOND models for the transition from the MOND to Newtonian regimes are tested to infer whether an additional DM component is required or whether MOND alone can correctly describe the dynamics of the galaxies in our sample: (i) the "simple" MOND formula from Famaey \& Binney (2005); (ii) the "standard" formula (Sanders \& McGaugh 2002); (iii) the Bekenstein's "toy" formula (Bekenstein 2004), as proposed in the context of his $\mathrm{TeV} e \mathrm{~S}$, which is a new relativistic gravitational theory that is an attempt to produce a framework for MOND consistent with General Relativity. The expressions, in terms of the Newtonian circular velocity and the radius, for the circular velocity obtained with MOND using these three formulae are given in Samurović \& Ćirković (2008).

1. The "simple" MOND formula is given by

$$
\mu(x)=\frac{x}{1+x} .
$$

2. The "standard" MOND formula is given by

$$
\mu(x)=\frac{x}{\sqrt{1+x^{2}}} .
$$

3. The "toy" MOND model is described with

$$
\mu(x)=\frac{-1+\sqrt{1+4 x}}{1+\sqrt{1+4 x}} .
$$

The MOND formalism also includes an external field effect (EFE). Richtler et al. (2011) studied the external gravitational fields acting on Virgo and Coma galaxies and found that the EFE can be neglected for the galaxies in our sample because of the lack of bright galaxies in their vicinities. We therefore neglect the EFE in our analysis, except for NGC 1400, which lies close to NGC 1407 (see Sect. 4.1).

\section{Results and discussion}

We now present the results of our Jeans modeling for each galaxy in the sample for the Newtonian and MOND approaches, presenting the best-fitting mass-to-light ratio for each model. After establishing the values of the dynamical mass-to-light ratio, we compare them with the values based on the models found in the literature: for example, we use stellar mass-to-light ratio as a fitting parameter in the dynamical models for the NFW models below from which the DM importance follows. We use various SPS models and compare them to the dynamical stellar mass-to-light ratios obtained using the Jeans models as a plausibility cross-check. First, we use the paper by Bell \& de Jong (2001) to calculate the theoretical mass-to-light ratios based only on the visible matter using the colors found in the HyperLeda database $^{3}$ : the predicted theoretical values for the galaxies in the sample are given in Table 5. The values listed in Table 5 are thus stellar mass-to-light ratios of the galaxies in the sample. We have used the colors from the HyperLeda database: the $B-V$ colors were used, and then the mass-to-light ratio in the $B$-band for a given metallicity $(Z=0.02)$ was calculated by applying the fitting formulae from Bell \& de Jong (2001). The used colors were corrected for Galactic extinction (Schlegel et al. 1998). Our chosen value of the metallicity was based on the work of Casuso et al. (1996), who used the Lick index $\left(\mathrm{Mg}_{2}\right)$, as an indicator of metallicity, since the $\mathrm{Mg}_{2}$ index for all our galaxies is between $\sim 0.25$ and $\sim 0.30$ (from HyperLeda). From Fig. 3 of Casuso et al., one can conclude that $Z=0.02$ is an accurate approximation.

Seven different models with several initial mass functions (IMFs) were used as given in Table 5: we used the Bruzual \& Charlot model (Bruzual \& Charlot 2003, hereafter BC) with Salpeter IMF and the PEGASE ${ }^{4}$ model (Fioc \& Rocca-Volmerange 1997) again with Salpeter IMF. (For a thorough discussion about the IMF and the problems of its use in the modeling, see Matteucci 2012.) We also used newer models of Into \& Portinari (2013, hereafter IP13), who presented their color-mass-to-light relations that include an accurate implementation of the TP-AGB (thermally pulsating asymptotic giant branch) phase and the effects of interstellar dust; they considered realistic chemical evolution models and the role of the mass-metallicity relation and metallicity gradients, which are important for the outskirts of disc galaxies. The estimates of the mass-to-light ratio based on their paper were derived for composite stellar populations (CSP, convolutions of simple stellar populations and SSPs of different age and metallicity, according to a given star formation and chemical evolution), which again uses $B-V$ colors from HyperLeda, are given in Table 5 in Cols. 4-6. The estimate in Col. 4 is based on the exponential star formation history (SFH) models with the Kroupa (2001) IMF, and the values in the next two columns come from disc galaxy models based on the Kroupa and Salpeter IMF, respectively. A Kroupa IMF is adopted as a reference IMF for the MW type

\footnotetext{
3 http://leda.univ-lyon1.fr

4 http://www2 . iap. fr/users/fioc/PEGASE.html
} 
Table 5. Values of the predicted stellar mass-to-blue-light ratios in various theoretical models.

\begin{tabular}{cccccccc}
\hline \hline code & BC & PEGASE & IP13 & IP13 & IP13 & CvD12 & CvD12 \\
\hline IMF & Salpeter & Salpeter & Kroupa1 & Kroupa2 & Salpeter & var. & Kroupa MW \\
metallicity & 0.02 & 0.02 & $\leq 0.03$ & $\leq 0.03$ & $\leq 0.03$ & $\sim 0.02$ & $\sim 0.02$ \\
SFH & SSP & SSP & CSP & CSP & CSP & SSP & SSP \\
$(1)$ & $(2)$ & $(3)$ & $(4)$ & $(5)$ & $(6)$ & $(7)$ & $(8)$ \\
\hline NGC 1400 & 6.0 & 6.6 & 3.9 & 4.3 & 7.4 & - & - \\
NGC 1407 & 7.4 & 8.1 & 5.0 & 5.7 & 9.8 & - & - \\
NGC 2768 & 6.4 & 7.0 & 4.2 & 4.7 & 8.2 & 1.41 & 1.48 \\
NGC 3115 & 6.2 & 6.8 & 4.0 & 4.5 & 7.8 & - & - \\
NGC 3377 & 4.8 & 5.2 & 2.9 & 3.1 & 5.4 & 3.27 & 2.81 \\
NGC 4278 & 6.2 & 6.8 & 4.0 & 4.5 & 7.8 & 7.51 & 4.06 \\
NGC 4365 & 6.9 & 7.6 & 4.6 & 5.2 & 9.0 & - & - \\
NGC 4486 & 6.7 & 7.3 & 4.4 & 5.0 & 8.5 & 1.28 & 0.68 \\
NGC 4494 & 5.3 & 5.8 & 3.2 & 3.6 & 6.2 & - & - \\
NGC 5846 & 7.1 & 7.8 & 4.8 & 5.5 & 9.4 & 3.95 & 2.22 \\
\hline
\end{tabular}

Notes. Column (1): name of the galaxy. Column (2): SPS Bruzual \& Charlot (BC) model with Salpeter IMF for $Z=0.02$. Column (3): SPS PEGASE model with Salpeter IMF for $Z=0.02$. Column (4): the exponential SFH model with the Kroupa IMF (Into \& Portinari 2013). Column (5): the disc galaxy model based on the Kroupa IMF (Into \& Portinari 2013). Column (6): the disc galaxy model based on the Salpeter IMF (Into \& Portinari 2013). Column (7): Conroy \& van Dokkum (2012) model allowing for IMF variation. Column (8): Conroy \& van Dokkum (2012) model assuming a fixed MW (Kroupa) IMF. See text for details.

of galaxy (see Conroy \& van Dokkum 2012, hereafter CvD12, where their new population synthesis model is presented.) At the lowest dispersions and $[\mathrm{Mg} / \mathrm{Fe}]$ values, their derived IMF is consistent with the MW IMF. In Cols. 7 and 8 of Table 5, we provide the estimates of the mass-to-light ratio (converted to the $B$-band) for six galaxies in common with the sample analyzed by CvD12. This is based on the models that respectively allow for IMF variation and for fixed (Kroupa) MW IMF ${ }^{5}$. These models predict the values of the mass-to-light ratio in the inner parts of the galaxies (interior to $R_{\mathrm{e}} / 8$ ) and are usually well below the estimates based on other SPS models (with the exception of NGC 4278, see Table 5). In Table 5, we also listed SFH.

For all the galaxies, we show the best-fitting results for isotropic and cases based on $\beta_{\text {lit }}$. The case of moderate tangential anisotropies, $\beta=-0.20$, are not shown as explained above, but is taken into account when the range of allowed mass-to-light ratios are presented. For each galaxy, we indicate the Sérsic index and scale radius for the stellar component, $n_{*}$ and $a_{\mathrm{s}}^{*}$, respectively, used in the fitting procedures. In all the figures with the Jeans modeling below, the thick lines are for isotropic cases and the thin lines are for the fits made using anisotropy determined by $\beta_{\text {lit }}$. Since we are interested here in DM, which is expected to dominate in the outer regions of galaxies when GCs are fit with two values of the $\gamma$ parameter, we always use the value that characterizes the outer part, $\gamma_{\text {out }}$, for the NFW models unless indicated otherwise. We apply the same approach in the MOND modeling: when we show only one set of models for the whole galaxy, we always use $\gamma_{\text {out }}$ in the Jeans equation.

For each model for which we solve the Jeans equation, we calculate the $\chi^{2}$ statistic:

$\chi^{2}=\sum_{i=1}^{N}\left[\frac{p_{i}^{\mathrm{obs}}-p_{i}^{\mathrm{mod}}}{\Delta p_{i}^{\mathrm{obs}}}\right]^{2}$,

where $N$ is the number of data points, $p_{i}^{\text {obs }}$ are the observed data points, the model values are given with $p_{i}^{\text {mod }}$, and $\Delta p_{i}^{\text {obs }}$ are

\footnotetext{
5 For the conversion from the $r$-band to the $B$-band, we used the absolute $r$ magnitudes of the galaxies from Cappellari et al. (2013a).
}

the uncertainties on the observed values at the given radius. We find the best fits by minimizing the $\chi^{2}$, and in the plots below (Sects. 4.1-4.10), the best-fit models are presented. The bestfitting results were obtained using an adaptive grid technique and the goodness-of-fit for each model is given. The quoted errors are $1 \sigma$ uncertainties, unless noted otherwise. We anticipate here that these uncertainties are useful when compared to of our results based on the Jeans modeling with the predictions from the cosmological simulations. (See Fig. 24 where typical error bars for the virial concentration parameter for two classes of galaxies are plotted.) Our goal is to find the best fit for each model and then to discuss its features (in comparison with the SPS models, as mentioned above) to infer the contribution of DM in them.

\subsection{NGC 1400}

The basic observational data on NGC 1400 are given in Table 4. We note here that two different early-type classifications exist for NGC 1400: a face-on S0 galaxy (Jarrett et al. 2003) and an E0 galaxy (da Costa et al. 1998). The kinematics of NGC 1400 is given in Fig. 1.

In the fits below, the values of $n_{*}=4.0$ and $R_{\mathrm{e}}^{(\mathrm{fit})}=26.6$ arcsec are used (Saxton \& Ferreras 2010; see also Coccato et al. 2009). The Newtonian fits of NGC 1400 are presented in Fig. 8 in the upper panel for three cases: the inner part ("in", interior to $\sim 4 R_{\mathrm{e}}$ ), the outer part ("out", beyond $\sim 3-4 R_{\mathrm{e}}$ ), and the NFW model. The interval of the values that provide a successful fit for the inner part is $4.2<M / L_{B}<9.2$. Thus, in the inner region, DM is not necessary and the values of the mass-to-light ratios agree with the theoretical models of stellar populations (see Tables 5 and 6 ). Beyond $\sim 3 R_{\mathrm{e}}$, the mass-to-light ratio needs to be increased to fit the observed LOS velocity dispersion: the interval becomes $7.8<M / L_{B}<19.8$. These results would thus imply the existence of additional unseen matter by taking the tested SPS models into account. The isotropic (radially anisotropic) NFW model with stellar mass-to-light ratio, $M_{*} / L_{B}=5.5(6.5)$ with a DM halo characterized with $400 \operatorname{arcsec}$ and $\rho_{\mathrm{s}}=0.0025(0.0035) M_{\odot} \mathrm{pc}^{-3}$ can also provide a successful fit to the LOS observed velocity dispersion. The lower 
Table 6. Values of the fit of mass-to-light ratios based on the Jeans modeling for the Newtonian models.

\begin{tabular}{|c|c|c|c|c|c|c|c|c|c|}
\hline Name & $(2)$ & $\begin{array}{c}\text { Const. } M / L_{B} \\
\left(r<3 R_{\mathrm{e}}\right) \\
(3) \\
\end{array}$ & $\chi^{2}$ & $\begin{array}{c}\text { Const. } M / L_{B} \\
\begin{array}{c}\left(r>3 R_{\mathrm{e}}\right) \\
(5)\end{array}\end{array}$ & $\chi^{2}$ & $\begin{array}{c}\mathrm{NFW} \\
{\left[M_{*} / L_{B} ; r_{\mathrm{s}} ; \rho_{\mathrm{s}}\right]} \\
(7)\end{array}$ & $\chi^{2}$ & $\begin{array}{c}\left(M / L_{B}\right)^{5 R_{\mathrm{e}}} \\
(9) \\
\end{array}$ & $\begin{array}{l}\left(M / L_{B}\right)^{\mathrm{rvir}} \\
(10)\end{array}$ \\
\hline \multirow[t]{3}{*}{ N1400 } & 0.00 & $6.6 \pm 1.2$ & 2.28 & $12.1 \pm 2.3$ & 1.83 & $5.5 \pm 0.5 ; 400 \pm 100 ; 0.0025 \pm 0.0010$ & 0.85 & 11.0 & 39.73 \\
\hline & -0.20 & $5.4 \pm 1.2$ & 2.12 & $9.8 \pm 2.0$ & 1.68 & $5.0 \pm 0.5 ; 350 \pm 100 ; 0.0020 \pm 0.0010$ & 1.93 & 8.8 & 30.79 \\
\hline & $\beta_{\text {lit }}$ & $8.0 \pm 1.2$ & 3.26 & $16.8 \pm 3.0$ & 2.40 & $6.5 \pm 0.5 ; 400 \pm 100 ; 0.0035 \pm 0.0010$ & 0.70 & 15.3 & 57.82 \\
\hline \multirow[t]{3}{*}{ N1407 } & 0.00 & $7.2 \pm 0.8$ & 7.46 & $42.8 \pm 2.2$ & 10.91 & $8.0 \pm 2.0 ; 550 \pm 100 ; 0.0080 \pm 0.0020$ & 1.98 & 46.5 & 205.21 \\
\hline & -0.20 & $5.6 \pm 0.6$ & 6.40 & $38.8 \pm 2.8$ & 10.89 & $7.5 \pm 2.0 ; 550 \pm 100 ; 0.0070 \pm 0.0020$ & 2.02 & 40.1 & 177.07 \\
\hline & $\beta_{\text {lit }}$ & $10.0 \pm 1.0$ & 12.14 & $53.8 \pm 2.2$ & 10.90 & $8.0 \pm 2.0 ; 650 \pm 100 ; 0.0070 \pm 0.0020$ & 1.82 & 55.2 & 177.07 \\
\hline \multirow[t]{3}{*}{ N2768 } & 0.00 & $12.1 \pm 1.3$ & 1.22 & $12.1 \pm 1.3$ & 1.22 & $8.0 \pm 1.0 ; 300 \pm 100 ; 0.0045 \pm 0.0020$ & 1.50 & 12.5 & 11.55 \\
\hline & -0.20 & $9.8 \pm 1.2$ & 1.05 & $9.8 \pm 1.2$ & 1.05 & $7.5 \pm 1.0 ; 250 \pm 100 ; 0.0045 \pm 0.0020$ & 1.08 & 10.1 & 11.55 \\
\hline & $\beta_{\text {lit }}$ & $15.0 \pm 2.0$ & 2.46 & $15.0 \pm 2.0$ & 2.46 & $9.0 \pm 1.0 ; 400 \pm 100 ; 0.0050 \pm 0.0020$ & 1.24 & 18.6 & 13.00 \\
\hline \multirow[t]{3}{*}{ N3115 } & 0.00 & $13.8 \pm 1.0$ & 0.39 & $40.8 \pm 5.0$ & 4.36 & $13.5 \pm 0.5 ; 350 \pm 50 ; 0.0900 \pm 0.0200$ & 7.79 & 51.5 & 94.52 \\
\hline & -0.20 & $10.8 \pm 1.2$ & 0.22 & $37.0 \pm 4.0$ & 4.39 & $13.0 \pm 0.5 ; 350 \pm 50 ; 0.0800 \pm 0.0200$ & 7.69 & 46.4 & 83.66 \\
\hline & $\beta_{\text {lit }}$ & $14.4 \pm 2.4$ & 0.04 & $47.0 \pm 5.6$ & 4.14 & $13.0 \pm 0.5 ; 350 \pm 50 ; 0.1000 \pm 0.0200$ & 8.00 & 55.4 & 105.42 \\
\hline \multirow[t]{3}{*}{ N3377 } & 0.00 & $6.2 \pm 1.0$ & 1.45 & $9.6 \pm 1.8$ & 2.52 & $6.5 \pm 0.5 ; 450 \pm 150 ; 0.0035 \pm 0.0020$ & 1.35 & 9.7 & 14.64 \\
\hline & -0.20 & $5.0 \pm 1.0$ & 1.56 & $8.0 \pm 1.2$ & 2.43 & $6.2 \pm 0.3 ; 400 \pm 150 ; 0.0030 \pm 0.0015$ & 2.24 & 8.0 & 12.31 \\
\hline & $\beta_{\text {lit }}$ & $7.8 \pm 1.4$ & 0.76 & $12.8 \pm 2.4$ & 2.98 & $7.3 \pm 0.8 ; 400 \pm 150 ; 0.0070 \pm 0.0040$ & 1.27 & 13.0 & 31.25 \\
\hline \multirow[t]{3}{*}{ N4278 } & 0.00 & $13.6 \pm 2.2$ & 0.00 & $27.6 \pm 1.4$ & 5.57 & $9.5 \pm 3.5 ; 180 \pm 30 ; 0.0850 \pm 0.0300$ & 3.00 & 32.9 & 63.79 \\
\hline & -0.20 & $9.8 \pm 1.5$ & 0.04 & $23.2 \pm 1.2$ & 5.44 & $9.0 \pm 2.0 ; 150 \pm 30 ; 0.0750 \pm 0.0300$ & 3.78 & 27.0 & 56.02 \\
\hline & $\beta_{\text {lit }}$ & $18.0 \pm 2.2$ & 0.08 & $39.4 \pm 4.2$ & 8.11 & $9.5 \pm 1.5 ; 180 \pm 30 ; 0.0850 \pm 0.0150$ & 3.00 & 34.9 & 63.79 \\
\hline \multirow[t]{3}{*}{ N4365 } & 0.00 & $21.6 \pm 2.2$ & 0.14 & $67.2 \pm 9.4$ & 1.62 & $10.0 \pm 3.0 ; 250 \pm 100 ; 0.0500 \pm 0.0200$ & 0.12 & 97.5 & 117.0 \\
\hline & -0.20 & $16.2 \pm 2.0$ & 0.09 & $56.8 \pm 8.4$ & 1.56 & $7.0 \pm 2.0 ; 250 \pm 100 ; 0.0400 \pm 0.0200$ & 0.13 & 77.1 & 92.3 \\
\hline & $\beta_{\text {lit }}$ & $25.0 \pm 2.6$ & 0.64 & $81.2 \pm 11.4$ & 2.10 & $10.0 \pm 3.0 ; 250 \pm 100 ; 0.0550 \pm 0.0200$ & 0.24 & 112.8 & 125.2 \\
\hline \multirow[t]{3}{*}{ N4486 } & 0.00 & $19.4 \pm 1.6$ & 17.82 & $41.0 \pm 2.6$ & 16.40 & $6.5 \pm 1.5 ; 300 \pm 50 ; 0.0700 \pm 0.0100$ & 21.79 & 56.4 & 51.3 \\
\hline & -0.20 & $13.8 \pm 1.2$ & 15.88 & $27.6 \pm 2.0$ & 15.17 & $6.0 \pm 1.0 ; 250 \pm 50 ; 0.0700 \pm 0.0100$ & 21.71 & 34.6 & 28.7 \\
\hline & $\beta_{\text {lit }}$ & $25.0 \pm 2.0$ & 27.36 & $69.8 \pm 3.0$ & 16.51 & $7.5 \pm 1.0 ; 350 \pm 50 ; 0.0750 \pm 0.0100$ & 20.30 & 89.5 & 89.7 \\
\hline \multirow[t]{3}{*}{ N4494 } & 0.00 & $1.2 \pm 0.2$ & 1.66 & $3.9 \pm 0.6$ & 2.70 & $1.5 \pm 0.5 ; 600 \pm 200 ; 0.0040 \pm 0.0020$ & 0.85 & 5.9 & 33.51 \\
\hline & -0.20 & $0.8 \pm 0.2$ & 1.04 & $3.2 \pm 0.6$ & 2.53 & $1.5 \pm 0.5 ; 550 \pm 200 ; 0.0035 \pm 0.0020$ & 0.65 & 4.9 & 29.02 \\
\hline & $\beta_{\text {lit }}$ & $1.6 \pm 0.2$ & 3.75 & $5.0 \pm 0.7$ & 3.36 & $2.2 \pm 0.8 ; 650 \pm 200 ; 0.0035 \pm 0.0020$ & 0.37 & 6.9 & 29.02 \\
\hline \multirow[t]{6}{*}{ N5846 } & 0.00 & $7.0 \pm 1.4^{\mathrm{I}}$ & $\mathbf{0 . 0 0}$ & $53.6 \pm 7.8^{\mathrm{II}}$ & 0.65 & $9.0 \pm 2.0 ; 300 \pm 150 ; 0.0450 \pm 0.0200$ & 3.82 & 58.5 & 104.53 \\
\hline & 0.00 & $88.3 \pm 18.1^{\mathrm{III}}$ & 0.01 & & & & & & \\
\hline & -0.20 & $5.3 \pm 1.1^{\mathrm{I}}$ & $\mathbf{0 . 0 0}$ & $49.0 \pm 6.8^{\mathrm{II}}$ & 0.66 & $8.0 \pm 2.0 ; 250 \pm 150 ; 0.0600 \pm 0.0200$ & 3.76 & 53.4 & 140.98 \\
\hline & -0.20 & $79.6 \pm 15.4^{\mathrm{III}}$ & $\mathbf{0 . 0 0}$ & & & & & & \\
\hline & $\beta_{\text {lit }}$ & $8.8 \pm 1.8^{\mathrm{I}}$ & $\mathbf{0 . 0 0}$ & $62.8 \pm 8.8^{\mathrm{II}}$ & 0.55 & $8.5 \pm 1.5 ; 300 \pm 100 ; 0.0600 \pm 0.0200$ & 3.52 & 75.6 & 140.98 \\
\hline & $\beta_{\text {lit }}$ & $105.0 \pm 22.4^{\mathrm{III}}$ & 0.01 & & & & & & \\
\hline
\end{tabular}

Notes. Column (1): name of the galaxy. Column (2): $\beta$ parameter for three cases (isotropic, mildly tangential, and the literature-based, see text for details). Columns (3) and (4): Newtonian mass-follows-light models interior to $3 R_{\mathrm{e}}$ and $\chi^{2}$ values. For the galaxies denoted in italics (NGC 4365 and NGC 4486), the inner regions are interior to $1 R_{\mathrm{e}}$ and $0.35 R_{\mathrm{e}}$, respectively. Columns (5) and (6): Newtonian mass-follows-light models beyond $3 R_{\mathrm{e}}$ and $\chi^{2}$ values. Columns (7) and (8): NFW models for the whole galaxy and $\chi^{2}$ values. Stellar mass-to-light ratio $M_{*} / L_{B}, r_{\mathrm{s}}$, is in arcsecs, and $\rho_{\mathrm{s}}$ is in $M_{\odot} \mathrm{pc}^{-3}$. For galaxy NGC 5846, the regions where the fits were obtained were formed differently. For the constant mass-to-light models: "I" indicates the fit obtained interior to $\sim 2 R_{\mathrm{e}}$, "II" indicates the fit obtained between $\sim 2$ and $\sim 6 R_{\mathrm{e}}$, and "III" denotes the region beyond $\sim 6 R_{\mathrm{e}}$. Column (9): mass-to-light ratios at $5 R_{\mathrm{e}}$. Column (10): mass-to-light ratios at $r_{\mathrm{vir}}$. The best-fits in this Table and in Table 7 are highlighted in bold characters and denote the best model among the three mass models (Newtonian-noDM, Newtonian-DM, and MOND-noDM).

panel of Fig. 8 shows that the three MOND models for the transition from the MOND to Newtonian regimes can fit the LOS observed velocity dispersion without the need of DM, and the standard MOND model requires the highest mass-to-light ratio, $3.7<M / L_{B}<9.2$, whereas the simple MOND model provided a fit with $3.0<M / L_{B}<7.8$ and the lowest mass-to-light was obtained in the case of the toy model, $2.4<M / L_{B}<6.0$ (see Tables 7 and 5).

Because of the vicinity of a massive galaxy, NGC 1407, the galaxy NGC 1400 is a prime candidate for the study of the aforementioned EFE. This effect occurs because MOND is a non-linear theory and because it breaks the strong equivalence principle. This effect influences non-isolated systems (Sanders \& McGaugh 2002). We estimate the EFE as follows. First, we calculate the gravitational force per unit mass exerted by NGC 1407 on NGC 1400 (Famaey et al. 2007b):

$a_{\mathrm{ext}}=\frac{\left(G M_{\mathrm{vir}}^{b} a_{0}\right)^{1 / 2}}{d}$,

where $M_{\mathrm{vir}}^{b}$ is the baryonic virial mass. From Table 8, we estimate that $M_{\mathrm{vir}}^{b} \approx 7 \times 10^{11} M_{\odot}$, and $d$ is the distance between 
Table 7. Values of the of fit of mass-to-light ratios based on the Jeans modeling for the MOND models.

\begin{tabular}{|c|c|c|c|c|c|c|c|}
\hline $\begin{array}{c}\text { Name } \\
(1)\end{array}$ & $\begin{array}{l}\beta \\
(2) \\
\end{array}$ & $\begin{array}{l}\text { Standard } \\
\text { MOND } \\
(3)\end{array}$ & $\begin{array}{l}\chi^{2} \\
(4)\end{array}$ & $\begin{array}{l}\text { Simple } \\
\text { MOND } \\
(5)\end{array}$ & $\begin{array}{l}\chi^{2} \\
(6) \\
\end{array}$ & $\begin{array}{c}\text { Toy } \\
\text { MOND } \\
(7)\end{array}$ & $\begin{array}{l}\chi^{2} \\
(8)\end{array}$ \\
\hline NGC 1400 & $\begin{array}{r}0.00 \\
-0.20 \\
\beta_{\text {lit }}\end{array}$ & $\begin{array}{l}5.8 \pm 1.2 \\
4.7 \pm 1.0 \\
7.6 \pm 1.6\end{array}$ & $\begin{array}{l}0.87 \\
0.63 \\
2.16\end{array}$ & $\begin{array}{l}4.8 \pm 1.0 \\
3.8 \pm 0.8 \\
6.3 \pm 1.5\end{array}$ & $\begin{array}{l}0.44 \\
0.31 \\
1.39\end{array}$ & $\begin{array}{l}3.7 \pm 0.9 \\
3.0 \pm 0.6 \\
5.0 \pm 1.0\end{array}$ & $\begin{array}{l}\mathbf{0 . 4 2} \\
\mathbf{0 . 3 0} \\
1.25\end{array}$ \\
\hline NGC 1407 & $\begin{array}{r}0.00 \\
0.00 \\
-0.20 \\
-0.20 \\
\beta_{\text {lit }} \\
\beta_{\text {lit }}\end{array}$ & $\begin{array}{r}7.0 \pm 0.8^{i} \\
37.0 \pm 2.0^{\circ} \\
4.5 \pm 0.5^{i} \\
29.8 \pm 1.2^{\circ} \\
9.0 \pm 0.6^{i} \\
45.4 \pm 5.0^{\circ}\end{array}$ & $\begin{array}{l}5.75 \\
4.94 \\
4.91 \\
4.39 \\
8.92 \\
5.94\end{array}$ & $\begin{array}{r}5.6 \pm 0.6^{i} \\
27.0 \pm 3.6^{o} \\
4.0 \pm 0.4^{i} \\
22.2 \pm 2.8^{o} \\
7.4 \pm 0.8^{i} \\
35.6 \pm 4.8^{o}\end{array}$ & $\begin{array}{l}4.47 \\
3.94 \\
4.26 \\
3.76 \\
6.19 \\
4.68\end{array}$ & $\begin{array}{r}4.2 \pm 0.4^{i} \\
20.6 \pm 2.4^{o} \\
3.0 \pm 0.4^{i} \\
17.2 \pm 2.2^{o} \\
5.8 \pm 0.8^{i} \\
27.6 \pm 2.8^{o}\end{array}$ & $\begin{array}{l}3.97 \\
4.17 \\
3.90 \\
4.02 \\
4.99 \\
4.83\end{array}$ \\
\hline NGC 2768 & $\begin{array}{r}0.00 \\
-0.20 \\
\beta_{\text {lit }}\end{array}$ & $\begin{array}{r}7.6 \pm 1.2 \\
5.9 \pm 1.1 \\
10.2 \pm 2.0\end{array}$ & $\begin{array}{l}5.21 \\
6.37 \\
2.53\end{array}$ & $\begin{array}{l}5.6 \pm 1.0 \\
4.4 \pm 1.0 \\
7.8 \pm 1.6\end{array}$ & $\begin{array}{l}5.80 \\
6.70 \\
3.22\end{array}$ & $\begin{array}{l}4.4 \pm 0.8 \\
3.4 \pm 0.8 \\
6.0 \pm 1.0\end{array}$ & $\begin{array}{l}5.21 \\
5.92 \\
2.92\end{array}$ \\
\hline NGC 3115 & $\begin{array}{r}0.00 \\
0.00 \\
-0.20 \\
-0.20 \\
\beta_{\text {lit }} \\
\beta_{\text {lit }}\end{array}$ & $\begin{array}{r}12.0 \pm 2.8^{i} \\
35.4 \pm 5.2^{o} \\
9.5 \pm 2.3^{i} \\
31.6 \pm 4.8^{o} \\
13.2 \pm 1.9^{i} \\
41.6 \pm 5.4^{o}\end{array}$ & $\begin{array}{l}\mathbf{0 . 0 0} \\
\mathbf{5 . 2 6} \\
0.00 \\
5.46 \\
0.01 \\
4.68\end{array}$ & $\begin{array}{r}10.4 \pm 2.6^{i} \\
26.4 \pm 4.4^{o} \\
8.1 \pm 2.1^{i} \\
23.4 \pm 3.4^{o} \\
11.9 \pm 1.9^{i} \\
31.8 \pm 4.8^{o}\end{array}$ & $\begin{array}{l}0.01 \\
6.43 \\
0.00 \\
6.59 \\
0.00 \\
5.84\end{array}$ & $\begin{array}{r}8.1 \pm 2.1^{i} \\
20.0 \pm 3.2^{o} \\
6.3 \pm 1.7^{i} \\
17.6 \pm 3.0^{\circ} \\
9.4 \pm 2.4^{i} \\
23.8 \pm 3.8^{o}\end{array}$ & $\begin{array}{l}0.00 \\
6.52 \\
0.00 \\
6.64 \\
0.00 \\
6.00\end{array}$ \\
\hline NGC 3377 & $\begin{array}{r}0.00 \\
-0.20 \\
\beta_{\text {lit }}\end{array}$ & $\begin{array}{l}3.4 \pm 1.0 \\
2.6 \pm 0.8 \\
5.2 \pm 1.4\end{array}$ & $\begin{array}{l}0.80 \\
0.92 \\
0.46\end{array}$ & $\begin{array}{l}2.6 \pm 0.6 \\
2.2 \pm 0.4 \\
3.8 \pm 0.8\end{array}$ & $\begin{array}{l}0.58 \\
0.54 \\
0.41\end{array}$ & $\begin{array}{l}2.2 \pm 0.4 \\
1.8 \pm 0.4 \\
3.0 \pm 0.6\end{array}$ & $\begin{array}{l}0.46 \\
0.57 \\
0.40\end{array}$ \\
\hline NGC 4278 & $\begin{array}{r}0.00 \\
0.00 \\
-0.20 \\
-0.20 \\
\beta_{\text {lit }} \\
\beta_{\text {lit }}\end{array}$ & $\begin{array}{r}11.3 \pm 2.7^{i} \\
22.4 \pm 3.2^{\circ} \\
8.2 \pm 2.2^{i} \\
17.0 \pm 2.4^{o} \\
17.2 \pm 3.0^{i} \\
28.0 \pm 3.0^{\circ}\end{array}$ & $\begin{array}{l}0.65 \\
\mathbf{2 . 6 6} \\
0.01 \\
4.05 \\
0.01 \\
\mathbf{1 . 7 9}\end{array}$ & $\begin{array}{r}9.6 \pm 2.0^{i} \\
16.2 \pm 2.2^{\circ} \\
7.2 \pm 2.0^{i} \\
12.8 \pm 2.0^{\circ} \\
13.4 \pm 3.2^{i} \\
23.6 \pm 3.2^{o}\end{array}$ & $\begin{array}{l}0.93 \\
3.96 \\
0.91 \\
4.04 \\
0.38 \\
3.89\end{array}$ & $\begin{array}{r}7.8 \pm 2.6^{i} \\
12.4 \pm 2.0^{\circ} \\
5.6 \pm 3.0^{i} \\
9.8 \pm 1.4^{o} \\
11.6 \pm 3.0^{i} \\
19.4 \pm 3.0^{\circ}\end{array}$ & $\begin{array}{l}0.76 \\
3.86 \\
0.99 \\
3.91 \\
0.10 \\
3.29\end{array}$ \\
\hline$N G C 4365$ & $\begin{array}{r}0.00 \\
0.00 \\
-0.20 \\
-0.20 \\
\beta_{\text {lit }} \\
\beta_{\text {lit }}\end{array}$ & $\begin{array}{r}20.4 \pm 2.6^{i} \\
56.8 \pm 10.0^{o} \\
15.0 \pm 2.0^{i} \\
46.8 \pm 7.8^{\circ} \\
23.6 \pm 2.8^{i} \\
70.6 \pm 11.0^{o}\end{array}$ & $\begin{array}{l}0.23 \\
0.50 \\
0.40 \\
0.39 \\
0.30 \\
0.89\end{array}$ & $\begin{array}{r}17.2 \pm 2.2^{i} \\
43.2 \pm 7.6^{o} \\
12.6 \pm 1.6^{i} \\
35.4 \pm 7.0^{o} \\
20.2 \pm 2.6^{i} \\
54.4 \pm 10.2^{o}\end{array}$ & $\begin{array}{l}0.75 \\
0.26 \\
1.11 \\
0.21 \\
0.20 \\
0.49\end{array}$ & $\begin{array}{r}13.4 \pm 1.8^{i} \\
32.8 \pm 6.4^{o} \\
9.8 \pm 1.4^{i} \\
27.0 \pm 4.8^{o} \\
16.0 \pm 2.2^{i} \\
41.4 \pm 7.8^{o}\end{array}$ & $\begin{array}{l}1.14 \\
0.26 \\
1.50 \\
0.22 \\
0.35 \\
0.47\end{array}$ \\
\hline$N G C 4486$ & $\begin{array}{r}0.00 \\
-0.20 \\
\beta_{\text {lit }}\end{array}$ & $\begin{array}{l}20.0 \pm 0.6 \\
13.6 \pm 1.2 \\
28.8 \pm 4.8\end{array}$ & $\begin{array}{l}33.69 \\
32.31 \\
53.54\end{array}$ & $\begin{array}{l}15.4 \pm 0.6 \\
11.6 \pm 1.0 \\
25.8 \pm 1.6\end{array}$ & $\begin{array}{l}24.06 \\
22.00 \\
41.72\end{array}$ & $\begin{array}{l}15.4 \pm 1.6 \\
10.6 \pm 1.0 \\
18.4 \pm 1.2\end{array}$ & $\begin{array}{l}24.36 \\
\mathbf{2 0 . 3 6} \\
30.94\end{array}$ \\
\hline NGC 4494 & $\begin{array}{r}0.00 \\
-0.20 \\
\beta_{\text {lit }}\end{array}$ & $\begin{array}{l}2.2 \pm 0.4 \\
1.6 \pm 0.2 \\
2.4 \pm 0.2\end{array}$ & $\begin{array}{l}\mathbf{0 . 0 5} \\
0.13 \\
1.54\end{array}$ & $\begin{array}{l}1.6 \pm 0.4 \\
1.2 \pm 0.2 \\
1.8 \pm 0.4\end{array}$ & $\begin{array}{l}0.10 \\
\mathbf{0 . 1 0} \\
1.08\end{array}$ & $\begin{array}{l}1.2 \pm 0.2 \\
1.0 \pm 0.2 \\
1.4 \pm 0.2\end{array}$ & $\begin{array}{l}0.20 \\
0.42 \\
1.34\end{array}$ \\
\hline NGC 5846 & $\begin{array}{r}0.00 \\
0.00 \\
-0.20 \\
-0.20 \\
\beta_{\text {lit }} \\
\beta_{\text {lit }}\end{array}$ & $\begin{array}{r}6.7 \pm 1.5^{i} \\
55.2 \pm 6.6^{o} \\
5.1 \pm 1.1^{i} \\
50.0 \pm 7.4^{o} \\
8.5 \pm 1.9^{i} \\
66.0 \pm 8.6^{o}\end{array}$ & $\begin{array}{l}0.00 \\
2.86 \\
0.00 \\
2.74 \\
0.00 \\
3.21\end{array}$ & $\begin{array}{r}5.7 \pm 1.4^{i} \\
44.8 \pm 6.0^{\circ} \\
4.2 \pm 1.2^{i} \\
40.2 \pm 5.6^{\circ} \\
7.3 \pm 1.7^{i} \\
54.2 \pm 8.0^{\circ}\end{array}$ & $\begin{array}{l}0.00 \\
2.59 \\
0.00 \\
2.56 \\
0.00 \\
2.69\end{array}$ & $\begin{array}{r}4.4 \pm 1.1^{i} \\
34.2 \pm 4.6^{o} \\
3.3 \pm 0.8^{i} \\
30.6 \pm 4.2^{o} \\
5.7 \pm 1.4^{i} \\
41.6 \pm 5.8^{o}\end{array}$ & $\begin{array}{l}0.00 \\
2.70 \\
0.00 \\
2.68 \\
0.00 \\
2.76\end{array}$ \\
\hline
\end{tabular}

Notes. Column (1): name of the galaxy. Column (2): $\beta$ parameter. Columns (3) and (4): standard MOND models and $\chi^{2}$ values. Columns (5) and (6): simple MOND models and $\chi^{2}$ values. Columns (7) and (8): toy MOND models and $\chi^{2}$ values. For all the MOND models, " $i$ " indicates interior region (interior to $\sim 3 R_{\mathrm{e}}$ ) and " $o$ " denotes the outer region (beyond $\sim 3 R_{\mathrm{e}}$ ). Two exceptions are for the galaxies denoted in italics (NGC 4365 and NGC 4486): their inner regions are interior to $1 R_{\mathrm{e}}$ and $0.35 R_{\mathrm{e}}$, respectively. The best-fits in this Table and in Table 6 are highlighted in bold characters and denote the best model among the three mass models (Newtonian-noDM, Newtonian-DM, and MOND-noDM).

the two objects. This estimate is based on the baryonic, stellar component for which we take the highest possible value to maximize the influence of the stellar content, $M / L_{B} \sim 10$ (see Tables 5-7). We, thus, take that the stellar contribution is $\sim 6 \%$. 
Table 8. Central velocity dispersion, the estimated dynamical mass, $M_{\mathrm{dyn}}$, the estimated virial mass, $M_{\mathrm{vir}}$, and the concentration parameter, $c_{\mathrm{vir}}$, of the galaxies in the sample.

\begin{tabular}{ccccr}
\hline \hline Name & $\begin{array}{c}\sigma_{0} \\
{\left[\mathrm{~km} \mathrm{~s}^{-1}\right]}\end{array}$ & $\begin{array}{c}M_{\text {dyn }} \\
{\left[M_{\odot}\right]}\end{array}$ & $\begin{array}{c}M_{\text {vir }} \\
{\left[M_{\odot}\right]}\end{array}$ & \multicolumn{1}{c}{$c_{\text {vir }}$} \\
$(1)$ & $(2)$ & $(3)$ & $(4)$ & \multicolumn{1}{c}{$(5)$} \\
\hline NGC 1400 & $251.5 \pm 4.4^{\mathrm{L}}$ & $(2.88 \pm 0.10) \times 10^{11}$ & $1.05_{-0.57}^{+0.59} \times 10^{12}$ & $5.38_{-1.24}^{+0.87}$ \\
NGC 1407 & $270.6 \pm 6.1^{\mathrm{L}}$ & $(7.74 \pm 0.34) \times 10^{11}$ & $1.17_{-0.42}^{+0.43} \times 10^{13}$ & $7.53_{-1.03}^{+0.82}$ \\
NGC 2768 & $202.3 \pm 10.1^{\mathrm{C}}$ & $(4.55 \pm 0.47) \times 10^{11}$ & $6.02_{-3.03}^{+3.28} \times 10^{11}$ & $6.35_{-1.32}^{+0.99}$ \\
NGC 3115 & $266.9 \pm 4.3^{\mathrm{L}}$ & $(3.11 \pm 0.10) \times 10^{11}$ & $1.39_{-4.00}^{+5.61} \times 10^{12}$ & $18.07_{-2.27}^{+1.95}$ \\
NGC 3377 & $145.6 \pm 7.3^{\mathrm{C}}$ & $(5.83 \pm 0.60) \times 10^{10}$ & $2.28_{-1.82}^{+1.96} \times 10^{11}$ & $7.03_{-2.89}^{+1.61}$ \\
NGC 4278 & $248.3 \pm 12.4^{\mathrm{C}}$ & $(1.79 \pm 0.18) \times 10^{11}$ & $5.89_{-2.65}^{+2.68} \times 10^{11}$ & $17.35_{-3.14}^{+2.31}$ \\
NGC 4365 & $255.9 \pm 12.8^{\mathrm{C}}$ & $(5.02 \pm 0.51) \times 10^{11}$ & $3.92_{-2.34}^{+1.95} \times 10^{12}$ & $14.48_{-3.79}^{+2.08}$ \\
NGC 4486 & $314.1 \pm 15.7^{\mathrm{C}}$ & $(7.53 \pm 0.77) \times 10^{11}$ & $4.70_{-2.30}^{+3.30} \times 10^{12}$ & $16.77_{-1.06}^{+0.95}$ \\
NGC 4494 & $154.2 \pm 7.7^{\mathrm{C}}$ & $(1.15 \pm 0.12) \times 10^{11}$ & $1.29_{-8.24}^{+8.96} \times 10^{12}$ & $5.82_{-1.12}^{+1.12}$ \\
NGC 5846 & $231.7 \pm 11.6^{\mathrm{C}}$ & $(4.34 \pm 0.45) \times 10^{11}$ & $6.56_{-3.55}^{+3.61} \times 10^{12}$ & $14.99_{-3.43}^{+2.36}$ \\
\hline
\end{tabular}

Notes. The central velocity dispersion were taken from the HyperLeda catalog ("L") and Cappellari et al. (2013b; "C"); the dynamical mass is calculated as given in Sect. 4. The concentration parameter is calculated as given in Sect. 3.

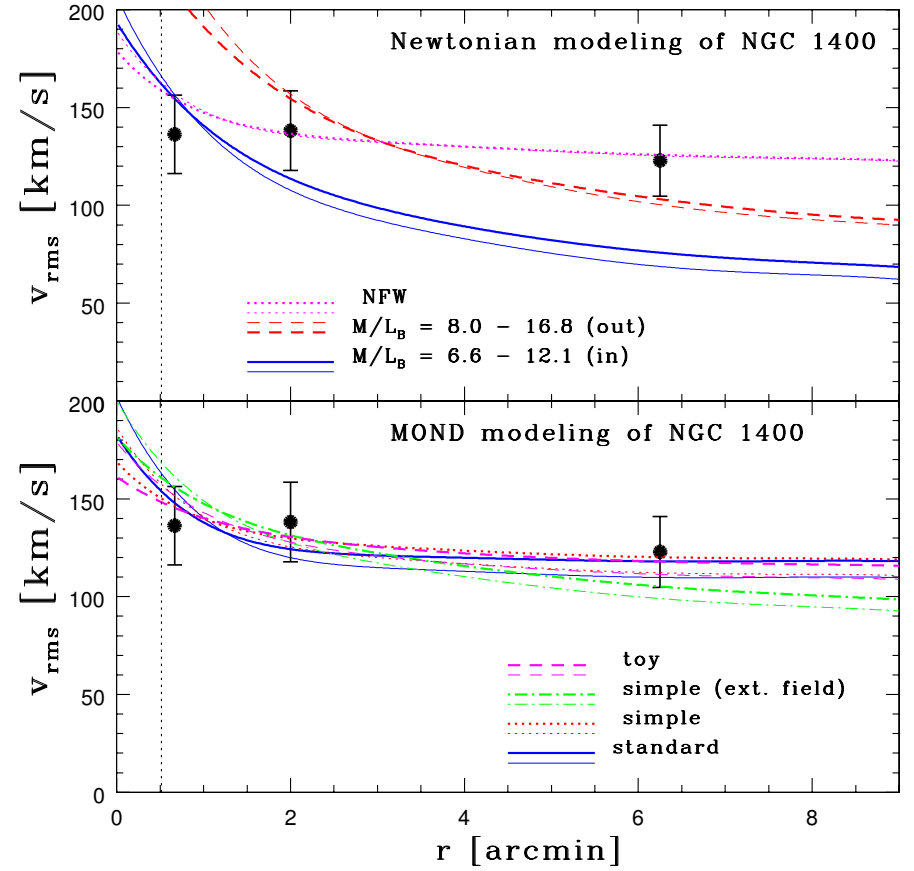

Fig. 8. Jeans Newtonian and MOND modeling of the projected velocity dispersion of NGC 1400 . The thick lines are for isotropic cases and the thin lines are for fits based on $\beta_{\text {lit }}$. Upper panel: for the Newtonian approach, there are two regions where the fitting was performed: inner region (interior to $\sim 3-4 R_{\mathrm{e}}$ ) and outer region (beyond $\sim 3 R_{\mathrm{e}}$ ). The thick solid blue line is for $M / L_{B}=6.6$ (inner region) and the thick dashed red line is for $M / L_{B}=12.1$ (outer region). The thin solid blue line is for $M / L_{B}=8.0$ (inner region) and the thin red dashed line $M / L_{B}=16.8$ (outer region). The NFW models with $M_{*} / L_{B}=5.5(6.5), r_{\mathrm{s}}=400$ arcsec, and $\rho_{\mathrm{s}}=0.0025(0.0035) M_{\odot} \mathrm{pc}^{-3}$ are plotted with the thick (thin) dotted magenta line. Lower panel: isotropic MOND models of the projected velocity dispersion of NGC 1400 for three interpolation functions $\mu(r)$. The standard MOND model is presented with the thick (thin) solid blue lines for $M / L_{B}=5.8$ (7.6); the simple MOND model is presented with the thick (thin) dotted red lines for $M / L_{B}=4.8(6.3)$, and the toy MOND model is presented with the thick (thin) dashed red lines for $M / L_{B}=3.7$ (5.0). The green dot-dashed lines are for the simple MOND model with the external field (see text for details). In both panels, one effective radius is indicated by a vertical dotted line.
Since both galaxies are at the same distance from us (see Table 4) and are separated by $\sim 12$ arcmin (see Spolaor et al. 2008), we use $d=93.6 \mathrm{kpc}$. Thus, we calculate that $a_{\mathrm{ext}}=0.28 a_{0}$. (This is the upper limit of the acceleration due to the EFE.) We tested the "simple" MOND model, where estimates of the mass-to-light ratio are intermediate for three MOND models for the transition from the MOND to Newtonian regimes (see Table 7) and apply the correction due to the EFE. We find that this effect does not significantly influence our results obtained in its absence. More precisely, for the isotropic case, the best-fitting mass-tolight ratio is $M / L_{B}=5.7 \pm 1.1$ for which $\chi^{2}=2.04$, and for the radially anisotropic case, the best-fitting case is obtained using $M / L_{B}=7.4 \pm 1.4$ (with $\chi^{2}=3.59$ ). Both fits are shown in Fig. 8. It can be seen that anisotropic case cannot fit the outermost observed point. To achieve this, one needs to increase the mass-to-light ratio to $M / L_{B} \sim 10$ (which is not shown). Thus, the inclusion of the EFE requires a small increase of the massto-light ratio, but such estimates are still consistent with the no dark-matter hypothesis, or with its small contribution. Because the EFE for other galaxies in the sample is smaller, we neglect it in our analysis below.

The stellar mass-to-light ratios inferred from the Newtonian mass-follows-light in the inner part agree with the tested SPS models. We note again that the predictions of the mass-to-light ratio based on the three MOND models for the transition from the MOND to Newtonian regimes are lower, and especially, the simple and toy models predict much lower values. The massto-light ratios found in the outer parts (beyond $\sim 3 R_{\mathrm{e}}$ ) in the Newtonian mass-follows-light approach imply the existence of DM there.

\subsection{NGC 1407}

The object NGC 1407 is a massive E0 galaxy and is the brightest galaxy in the Eridanus group, the second brightest galaxy in this group is NGC 1400. The basic observational data on NGC 1407 are given in Table 4. The kinematics of GCs of NGC 1407 are given in Fig. 1.

For the fits below, we use the values of $n_{*}=8.3$ and $R_{\mathrm{e}}^{\text {(fit) }}=67.4 \operatorname{arcsec}$ (Saxton \& Ferreras 2010; see also Coccato et al. 2009). As shown, the fits were done in an inner and outer 


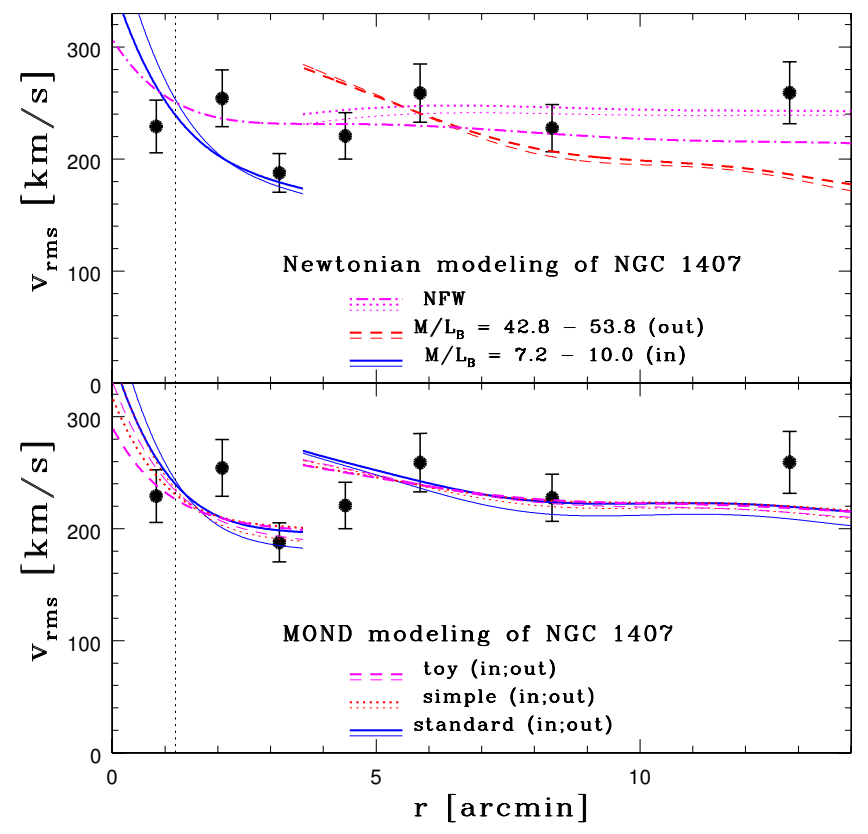

Fig. 9. Jeans Newtonian and MOND modeling of the projected velocity dispersion of NGC 1407. The thick lines are for isotropic cases, and the

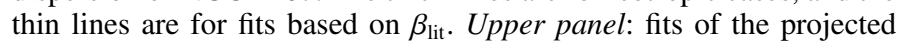
velocity dispersion of NGC 1407 using the Newtonian approach for the inner ("in", solid lines) and outer ("out", dashed lines) part. In the inner part, the thick blue line is for $M / L_{B}=7.2$, and the thin blue line is for $M / L_{B}=10.0$. In the outer part, the thick red line is for $M / L_{B}=$ 42.8 , and the thin red line is for $M / L_{B}=53.8$. The NFW models with $\left.M_{*} / L_{B}=8.0, r_{\mathrm{s}}=550(650) \operatorname{arcsec}, \rho_{\mathrm{s}}=0.0080(0.0070)\right) M_{\odot} \mathrm{pc}^{-3}$ are shown with the thick (thin) dotted magenta lines. The thick dot-dashed magenta line is for the isotropic NFW model for which $M / L_{B}=6.5$, $r_{\mathrm{s}}=400 \operatorname{arcsec}$ and $\rho_{\mathrm{s}}=0.0070 M_{\odot} \mathrm{pc}^{-3}$. Lower panel: again, the galaxy is split into its inner ("in") and outer ("out") part. The values of the mass-to-light ratio of the fits are as follows: for the standard MOND model, $M / L_{B}=7.0$ (37.0) for the inner (outer) part for the isotropic case and $M / L_{B}=9.0$ (45.4) for the inner (outer) part for the anisotropic case based on $\beta_{\text {lit }}$. For the simple MOND model, $M / L_{B}=5.6$ (27.0) for the inner (outer) part for the isotropic case, and $M / L_{B}=7.4$ (35.6) for the inner (outer) part for the anisotropic case based on $\beta_{\text {lit }}$. For the toy MOND model, $M / L_{B}=4.2$ (20.6) for the inner (outer) part for the isotropic case and $M / L_{B}=5.8$ (27.6) for the inner (outer) part for the anisotropic case based on $\beta_{\text {lit }}$. In both panels, one effective radius is indicated by a vertical dotted line.

region, and we note that two different values of the $\gamma$ parameter that characterize the radial surface density were used for this task: $\gamma=0.94$ (inner region) and $\gamma=2.55$ (outer region), see Fig. 6. The example of NGC 1407 represents another case of a galaxy where ordinary, stellar matter appears to dominate in the inner regions. The Newtonian fits of NGC 1407 are presented in Fig. 9 in the upper panel. The velocity dispersion of NGC 1407 can be fit with a constant mass-to-light ratio without anisotropies (as seen in the value $M / L_{B}=7.2$ ) and the anisotropic case (based on $\beta_{\text {lit }}$ with $M / L_{B}=10.0$ ). Taking the values from Table 6 into account, the interval of the values that provides a successful fit for the inner part, interior to $\sim 3 R_{\mathrm{e}}$ is $5.0<M / L_{B}<11.0$ and the observations of the GCs in the inner region thus show that $\mathrm{DM}$ is not necessary (or that the contribution is small) when one uses the tested SPS models. Beyond $\sim 3 R_{\mathrm{e}}$, the interval of the allowed mass-to-light ratio becomes $36.0<M / L_{B}<56.0$. (The two fits from the given range are shown in the upper panel of Fig. 9: $M / L_{B}=42.8$ and $M / L_{B}=53.8$, respectively). To fit the outermost point, one requires a higher mass-to-light ratio, $M / L_{B} \sim 100$. These estimates suggest the existence of additional unseen matter by considering all the stellar population models (see Tables 6 and 5). The NFW models with stellar mass-tolight ratio, $M_{*} / L_{B}=8.0$, with a DM halo characterized with $r_{\mathrm{s}}=550(650)$ arcsec and $\rho_{\mathrm{s}}=0.0080(0.0070) M_{\odot} \mathrm{pc}^{-3}$ also provide a successful fit to the LOS observed velocity dispersion. To check whether the NFW models can provide a fit throughout the whole galaxy, we search for a best fit based on a single parameter $\alpha$, which is valid in both the inner and the outer region. It was found that $\alpha=2.28$, and the best fit $\left(\chi^{2}=10.80\right)$ for the isotropic case with $M_{*} / L_{B}=6.5, r_{\mathrm{s}}=400 \mathrm{arcsec}$, and $\rho_{\mathrm{s}}=0.0070 M_{\odot} \mathrm{pc}^{-3}$ is plotted with the thick dot-dashed line in Fig. 9. As can be seen, although the quality of the fit is poorer with respect to the fit found in the outer region, the NFW models (also the two anisotropic case not shown here) can provide a fit for NGC 1407. In the lower panel of Fig. 9 the three MOND models for the transition from the MOND to Newtonian regimes are plotted. All three models can fit the LOS observed velocity dispersion without the need of DM in the inner part ("in"), out to $\sim 5 \operatorname{arcmin}\left(=4.2 R_{\mathrm{e}}\right)$. The standard MOND model requires the highest mass-to-light ratio, $4.0<M / L_{B}<9.6$ (the upper limit is close to the value predicted by the IP13 Salpeter model), whereas the simple MOND model provided a fit with $3.6<M / L_{B}<8.2$, and the lowest mass-to-light was obtained in the case of the toy model, $2.6<M / L_{B}<6.6$ (see Tables 7 and 5). None of the three MOND models for the transition from the MOND to Newtonian regimes can provide a fit beyond $4.2 R_{\mathrm{e}}$ without the additional, unseen component, and a set of fits in this region ("out") suggests the existence of additional, unseen matter there. The allowed intervals of the mass-to-light ratio in the outer part of NGC 1407 are $28.6<M / L_{B}<50.4$ (standard MOND model), $19.4<M / L_{B}<40.4$ (simple MOND model), and $15.0<M / L_{B}<30.4$ (toy MOND model). Both massfollows-light and MOND models imply the rise of the mass-tolight ratio and, thus, the existence of DM in the outer parts in the galaxy NGC 1407.

D12 quote the mass-to-light ratio based on the use of GCs interior to $5 R_{\mathrm{e}}, M / L_{B}=19 \pm 2$, which is consistent with our results related to Newtonian modeling based on the constant massto-light ratio. Namely, our values in the interior of NGC 1407 are lower (see Table 6), but they are related to a region interior to $\sim 3 R_{\mathrm{e}}$. Our values in the outer region are higher, but they are related to the data points beyond $\sim 5 R_{\mathrm{e}}$. To fit the point at $4.42 \operatorname{arcmin}\left(=3.68 R_{\mathrm{e}}\right)$ using the Newtonian mass-follows-light assumption, we used $M / L_{B}=28.0 \pm 11.2$ (which includes the uncertainty related to the anisotropy, i.e., all three cases of the $\beta$ parameter were tested). This value (for which the plot is not shown) is consistent with that obtained by D12.

\subsection{NGC 2768}

For NGC 2768, we again face an ambiguity with respect to its classification: de Vaucouleurs et al. (1991) classified it as E6 galaxy, whereas Sandage \& Bedke (1994) classified it as $\mathrm{SO}_{1 / 2}$. The object NGC 2768 is at the center of the loose group, which is cataloged as Lyon group of galaxies (LGG) 167 (Garcia 1993). Crocker et al. (2008) listed all known galaxies within $400 \mathrm{kpc}$ in their Table 3 that may have recently interacted with NGC 2768. They listed eight objects, and the nearest galaxy is at $72 \mathrm{kpc}$, and the farthest one is at the distance of $317 \mathrm{kpc}$. The basic observational data on NGC 2768 are given in Table 4 . The kinematics of NGC 2768 are given in Fig. 2.

For the fits below, we use the values of $n_{*}=3.3$ and $R_{\mathrm{e}}^{(\mathrm{fit})}=81.8 \operatorname{arcsec}$ (Krajnović et al. 2013). In the case of 


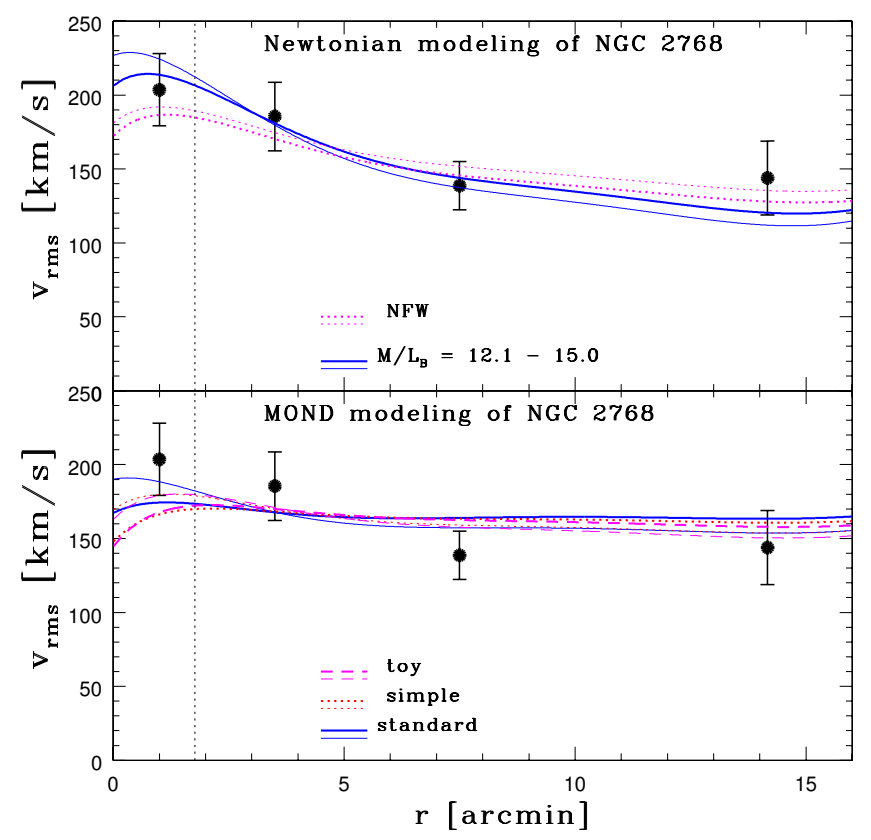

Fig. 10. Jeans Newtonian and MOND modeling of the projected velocity dispersion of NGC 2768 . The thick lines are for isotropic cases, and the thin lines are for fits based on $\beta_{\text {lit }}$. Upper panel: in the Newtonian approach, the mass-follows-light models are plotted with the thick (thin) solid blue line for $M / L_{B}=12.1$ (15.0), and the NFW models with $M_{*} / L_{B}=8.0(9.0)$ are $r_{\mathrm{s}}=300(400)$ arcsec and $\rho_{\mathrm{s}}=$ $0.0045(0.0050) M_{\odot} \mathrm{pc}^{-3}$, which are plotted with the thick (thin) dotted magenta line. Lower panel: MOND models of the projected velocity dispersion of NGC 2768 for three interpolation functions $\mu(r)$ for both isotropic and anisotropic case (based on $\beta_{\text {lit }}$ ). The standard MOND model is presented with the thick (thin) solid blue line for $M / L_{B}=$ 7.6 (10.2); the simple MOND model is presented with the thick (thin) dotted red line for $M / L_{B}=5.6$ (7.8); and the toy MOND model is presented with the thick (thin) dashed red line for $M / L_{B}=4.4$ (6.0). In both panels, one effective radius is indicated by a vertical dotted line.

NGC 2768, one again encounters, using mass-follows-light Newtonian models, a galaxy for which the velocity dispersion can be fit with a single $M / L_{B}$ value. The interval of the allowed mass-to-light ratio for the mass-follows-light Newtonian models is $8.6<M / L_{B}<17.0$. Here, the PEGASE and IP13 Salpeter models provide the best agreement with the lower estimates obtained from the Jeans models. The fits of NGC 2768 presented in Fig. 10 in the upper panel are the fits obtained using $M / L_{B}=12.1$ (15.0) for the isotropic (anisotropic case based on $\left.\beta_{\text {lit }}\right)$. The anisotropic value is higher than the values found using tested SPS models, which may imply the existence of some DM in NGC 2768. The NFW models with stellar mass-to-light ratio, $M_{*} / L_{B}=8.0(9.0)$ valid for the isotropic (anisotropic case based on $\beta_{\text {lit }}$ ) with a DM halo characterized with $r_{\mathrm{s}}=300$ (400) arcsec and $\rho_{\mathrm{s}}=0.0045(0.0050) M_{\odot} \mathrm{pc}^{-3}$ also provide successful fits to the LOS observed velocity dispersion. In the lower panel of Fig. 10, the three MOND models for the transition from the MOND to Newtonian regimes are plotted. All three models can fit the LOS observed velocity dispersion throughout the whole galaxy. (We note that the outermost point at $\sim 14$ armin can only be fit with the anisotropic models based on $\beta_{\text {lit. }}$.) The standard MOND model requires the highest mass-to-light ratio, $4.8<M / L_{B}<12.2$; the simple MOND model provided a fit with $3.4<M / L_{B}<9.4$. The lowest mass-to-light was obtained in the case of the toy model, $2.6<M / L_{B}<7.0$ (see Tables 7 and 5). The outermost points can be fit using MOND only for the anisotropic model based on $\beta_{\text {lit }}$ and for the standard $\mu(x)$ formula. The values obtained for the MOND models are in reasonable agreement with the BC, PEGASE, and IP13 models. The values of the mass-to-light ratio found in CvD12 are too low for all three MOND models for the transition from the MOND to Newtonian regimes. As mentioned above, none of the three isotropic MOND models can provide a fit to the two points beyond $\sim 5$ arcmin $\left(=3 R_{\mathrm{e}}\right)$ because all three fits overestimate the LOS observed velocity dispersion (a case similar to that encountered in Salinas et al. (2012), who analyzed the galaxy NGC 7507 using the standard and simple MOND models).

\subsection{NGC 3115}

The object NGC 3115 is a massive S0 galaxy and is the only field galaxy in the sample, which is the closest galaxy in the sample. The basic observational data on NGC 3115 are given in Table 4. The kinematics of GCs of NGC 3115 are given in Fig. 2.

The values of $n_{*}=4.4$ and $R_{\mathrm{e}}^{(\mathrm{fit})}=103 \operatorname{arcsec}(\mathrm{Hu} 2008)$ are used in the Jeans fits below. As shown, the fits were done in an inner and outer region and we note here that two different values of the $\gamma$ parameter that characterize the radial surface density were used for this task: $\gamma=1.34$ and steeper $\gamma=4.17$, see Fig. 6. Using Newtonian mass-follows-light models for NGC 3115, we can see that one cannot fit the LOS observed velocity dispersion with a single mass-to-light ratio throughout the whole galaxy (see the upper panel of Fig. 11). In the inner part $\left(\sim 3 R_{\mathrm{e}}\right)$, the values of the mass-to-light ratio are in the interval $9.6<M / L_{B}<16.8$, which implies little or no DM in a given region. In this case, the IP13 Salpeter models provide the best agreement with the lower values of the estimates obtained from the Jeans models (see Table 5), and based on the estimates of the tested SPS models, there appears to be a DM present even in the inner regions of NGC 3115 . Beyond $\sim 3 R_{\mathrm{e}}$ in the outer region of NGC 3115 , the increase in the mass-to-light ratio is required to fit the outermost point at $\sim 5 R_{\mathrm{e}}$; the allowed interval for the mass-to-light then becomes $33.0<M / L_{B}<52.6$, which indicates the copious amount of DM in the outer parts of NGC 3115 (see Tables 6 and 5). The two NFW models (which practically overlap) presented in Fig. 11 with a stellar mass-tolight ratio, $M_{*} / L_{B}=13.5$ (13.0) with a DM halo characterized with $r_{\mathrm{s}}=350 \operatorname{arcsec}$ and $\rho_{\mathrm{s}}=0.0900(0.1000) M_{\odot} \mathrm{pc}^{-3}$, can provide a successful fit to the LOS observed velocity dispersion. All three MOND models for the transition from the MOND to Newtonian regimes can provide a fit to the LOS observed velocity dispersion consistent with the lack of DM in the inner part ("in"), out to $\sim 3 \operatorname{arcmin}\left(=2 R_{\mathrm{e}}\right.$; based on $\gamma_{\text {in }}$ and shown in Fig. 11, see also Table 7). In the lower panel of Fig. 11, the three MOND models for the transition from the MOND to Newtonian regimes based on $\gamma_{\text {out }}$ are shown and are valid beyond $\sim 3 R_{\mathrm{e}}$. The standard MOND model as usual requires the highest mass-tolight ratio, $26.8<M / L_{B}<47.0$, the simple MOND model provided a fit with $20.0<M / L_{B}<36.6$, and the lowest mass-tolight ratio (marginally consistent with the stellar mass only) was obtained in the case of the toy model, $14.6<M / L_{B}<27.6$ (see Tables 7 and 5). None of the three MOND models for the transition from the MOND to Newtonian regimes (except isotropic and mildly tangentially anisotropic toy model) can provide a fit beyond $\sim 3 R_{\mathrm{e}}$ without $\mathrm{DM}$ and a set of fits in this outer region ("out") indicates the existence of additional, unseen matter there. Both Newtonian mass-follows-light and MOND models imply the rise of the mass-to-light ratio and, thus, the existence of DM in the outer parts of the galaxy NGC 3115. 


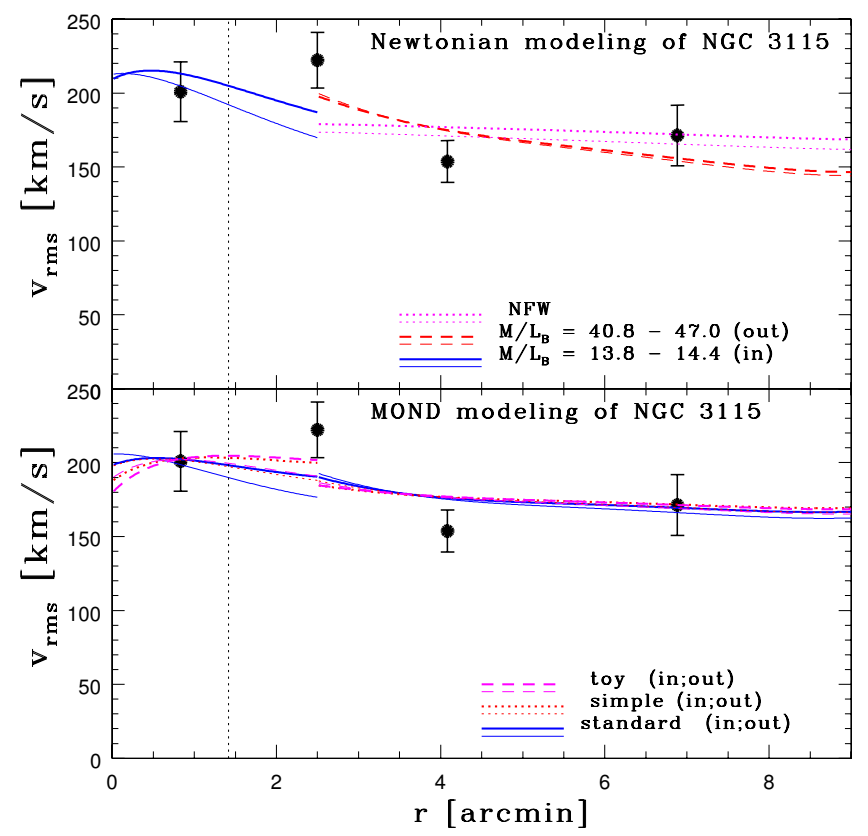

Fig. 11. Jeans Newtonian and MOND modeling of the projected velocity dispersion of NGC 3115. The thick lines are for isotropic cases and the thin lines are for fits based on $\beta_{\text {lit }}$. Upper panel: fits of the projected velocity dispersion of NGC 3115 using the Newtonian approach for the inner ("in", solid lines) and outer ("out", dashed lines) part. In the inner part, the thick blue line is for $M / L_{B}=13.8$, and the thin blue line is for $M / L_{B}=14.4$. In the outer part, the thick red line is for $M / L_{B}=40.8$, and the thin red line is for $M / L_{B}=47.0$. The NFW models with $M_{*} / L_{B}=13.5(13.0), r_{\mathrm{s}}=350 \operatorname{arcsec}$ and $\rho_{\mathrm{s}}=0.0900(0.1000) M_{\odot} \mathrm{pc}^{-3}$ are shown with the thick (thin) dotted magenta lines. Lower panel: the galaxy is split into its inner ("in") and outer ("out"). The values of the mass-to-light ratio of the fits are: for the standard MOND model, $M / L_{B}=12.0$ (35.4) for the inner (outer) part for the isotropic case, and $M / L_{B}=13.2$ (41.6) for the inner (outer) part for the anisotropic case based on $\beta_{\text {lit }}$. For the simple MOND model, $M / L_{B}=10.4$ (26.4) for the inner (outer) part for the isotropic case, and $M / L_{B}=11.9$ (31.8) for the inner (outer) part for the anisotropic case based on $\beta_{\text {lit. }}$. For the toy MOND model, $M / L_{B}=8.1$ (20.0) for the inner (outer) part for the isotropic case and $M / L_{B}=9.4$ (23.8) for the inner (outer) part for the anisotropic case based on $\beta_{\mathrm{lit}}$. In both panels, one effective radius is indicated by a vertical dotted line.

The galaxy NGC 3115 was analyzed by Emsellem et al. (1999), who used the Jeans models to infer the mass-to-light in the inner region (inside $\sim 1 \operatorname{arcmin}$ ) at $M / L_{B}=13.4$ (converted from the $V$-band to the $B$-band for the sake of comparison). Our results for the inner part of NGC 3115 agree with this result (see Table 6). We note that Emsellem et al. also provide predictions based on their three-integral models for the mass-to-light ratio at several effective radii. For their outermost point ( $5 \mathrm{arcmin}$, no observational data available), they predict $M / L_{B}=85.5$, which is higher than our highest predicted value in Newtonian massfollows-light approximation, at $M / L_{B}=70.6$. Given the still small number of observed tracers in the outermost regions of NGC 3115 and large uncertainties regarding the value of the velocity dispersion there, it is difficult to firmly establish the mass-to-light ratio in the outermost region. One can expect that new observations will clarify this. At the moment, however, one can conclude that NGC 3115 shows a significant increase of the mass-to-light ratio in its outer parts, which is most probably to the existence of the additional, unseen component.

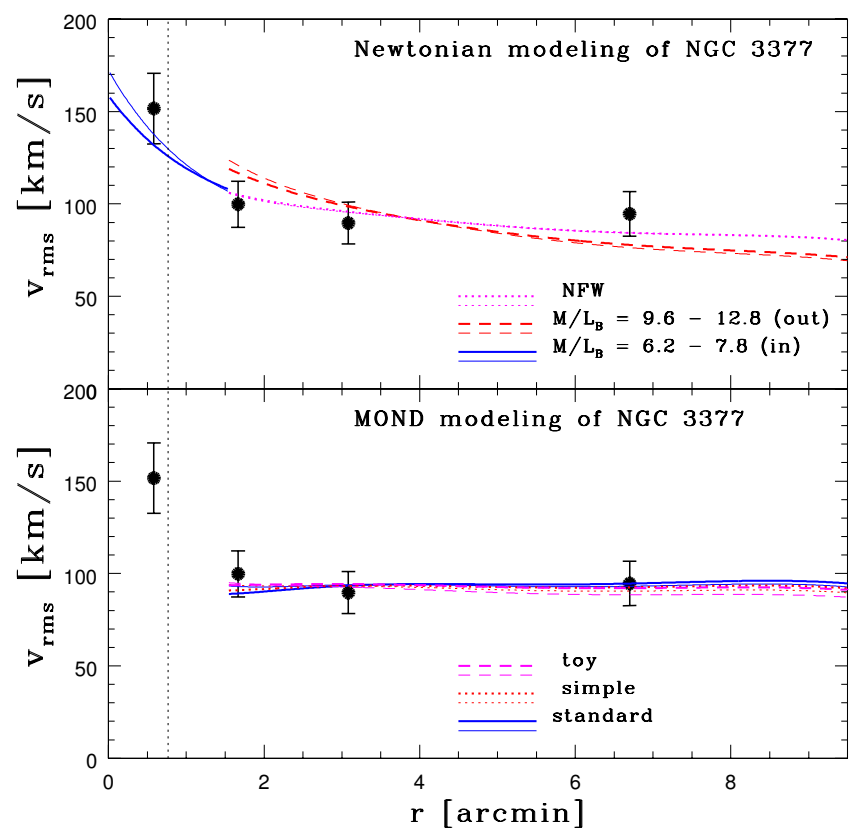

Fig. 12. Jeans Newtonian and MOND modeling of the projected velocity dispersion of NGC 3377. The thick lines are for isotropic cases and the thin lines are for fits based on $\beta_{\text {lit }}$. Upper panel: fits of the projected velocity dispersion of NGC 3377 using the Newtonian approach for the inner ("in", solid blue lines) and outer ("out", dashed red lines) part. In the inner part, the thick (thin) solid line is for $M / L_{B}=6.2$ (7.8). In the outer part, the thick (thin) dashed line is for $M / L_{B}=9.6(12.8)$. The NFW models, with $M_{*} / L_{B}=6.5(7.3), r_{\mathrm{s}}=450$ (400) arcsec, and $\rho_{\mathrm{s}}=0.0035(0.0070) M_{\odot} \mathrm{pc}^{-3}$ are shown with the thick (thin) dotted magenta lines. Lower panel: standard MOND model is presented with the thick (thin) solid blue line for $M / L_{B}=3.4$ (5.2); the simple MOND model is presented with the thick (thin) red dotted line for $M / L_{B}=2.6$ (3.8), and the toy MOND model is presented with the thick (thin) red dashed line for $M / L_{B}=2.2$ (3.0). In both panels, one effective radius is indicated by a vertical dotted line.

\subsection{NGC 3377}

The object NGC 3377 is a massive E6 galaxy in the Leo I group. The basic observational data on NGC 3377 are given in Table 4. The kinematics of GCs of NGC 3377 are given in Fig. 3.

The values of $n_{*}=5.0$ and $R_{\mathrm{e}}^{(\mathrm{fit})}=53.4 \operatorname{arcsec}($ Krajnović et al. 2013) are used in the Jeans fits below. As shown, the fits were done in an inner and outer region, and for this task, two different values of the $\gamma$ parameter that characterize the radial surface density were used: $\gamma=1.38$ (inner region) and $\gamma=1.93$ (outer region), see Fig. 6. Using Newtonian mass-follows-light models for NGC 3377, we find that one cannot fit the LOS observed velocity dispersion with a single mass-to-light ratio throughout the whole galaxy (see the upper panel of Fig. 12). In the inner part $\left(\sim 3 R_{\mathrm{e}}\right)$, the values of the mass-to-light ratio are in the interval $4.0<M / L_{B}<9.2$, and they are consistent with a no dark-matter hypothesis and BC, PEGASE and IP13 Salpeter SPS models (see Table 5). Beyond $\sim 3 R_{\mathrm{e}}$, in the outer region of NGC 3377, one needs to increase the mass-to-light ratio to fit the outermost point at $\sim 10 R_{\mathrm{e}}$. The allowed interval for the massto-light then becomes $6.8<M / L_{B}<15.2$, which indicates that there is DM in its outer parts (see Tables 6 and 5). To fit the outermost point, one needs $M / L_{B} \sim 20$. The NFW models presented in Fig. 12 with a stellar mass-to-light ratio, $M_{*} / L_{B}=6.5$ (7.3), with a DM halo characterized by $r_{\mathrm{s}}=450(400)$ arcsec and $\rho_{\mathrm{s}}=0.0035(0.0070) M_{\odot} \mathrm{pc}^{-3}$ also provide a successful fit to 
the LOS observed velocity dispersion. In the lower panel of Fig. 12, the three MOND models for the transition from the MOND to Newtonian regimes are plotted: Except for the innermost point at 1 arcmin, all three models can fit the LOS observed velocity dispersion without the need of DM. The highest mass-to-light ratio of the standard MOND model is in the interval $1.8<M / L_{B}<6.6$; the simple MOND model provided a fit with $1.8<M / L_{B}<4.6$, and as usual, the lowest mass-to-light was obtained in the case of the toy model, $1.4<M / L_{B}<3.6$ (see Tables 7 and 5). The estimates of the mass-to-light ratio obtained from the three MOND models for the transition from the MOND to Newtonian regimes are consistent with the estimates obtained using all the tested SPS models for the first two cases and with IP13 Kroupa and CvD12 models for the toy model.

The mass-to-light ratio $M / L_{B}=12 \pm 2$ for NGC 3377, which is based on PNe obtained by D12, agrees with our calculated value for the region beyond $\sim 3 R_{\mathrm{e}}$ (see Table 6 ).

\subsection{NGC 4278}

The object NGC 4278 is classified as an (E1-2) elliptical galaxy and is a member of the Coma I cloud. The basic observational data on NGC 4278 are given in Table 4. The kinematics of GCs of NGC 4278 are given in Fig. 3.

The values of $n_{*}=4.8$ and $R_{\mathrm{e}}^{(\mathrm{fit})}=31.6$ arcsec Krajnović et al. (2013) are used in the Jeans fits below. Again, the fits were done in an inner and outer region, and thus two different values of the $\gamma$ parameter that characterize the radial surface density were used: $\gamma=0.90$ (inner region) and $\gamma=1.87$ (outer region), see Fig. 7. The Newtonian mass-follows-light models imply the allowed interval for the mass-to-light ratio of $8.3<M / L_{B}<20.2$ in the inner region (interior to $\sim 3 R_{\mathrm{e}}$ ). We present the fits with $M / L_{B}=13.6$ for the isotropic case and $M / L_{B}=18.0$ for the anisotropic case based on $\beta_{\text {lit }}$ (see the upper panel of Fig. 13). Here, IP13 Salpeter and CvD12 models with a variation in IMF models provide the values of the mass-to-light ratio that are close to lower values of the estimates obtained from the Jeans models (see Table 5). This might indicate the presence of DM even in the inner parts $\left(\lesssim 3 R_{\mathrm{e}}\right)$ of NGC 4278 . Beyond $\sim 3 R_{\mathrm{e}}$, the velocity dispersion again rises (to approximately $200 \mathrm{~km} \mathrm{~s}^{-1}$ ), which implies the increase of the total cumulative mass a part of which must be dark: the allowed interval of the mass-to-light ratio in the outer region is $22.0<M / L_{B}<43.6$ (see Table 6). The values of the mass-tolight ratio are clearly incompatible with any theoretical model based on the visible matter (see Table 5). The fits obtained using the NFW model have the following parameters: $M_{*} / L_{B}=9.5$, $r_{\mathrm{s}}=180 \operatorname{arcsec}$ and $\rho_{\mathrm{s}}=0.0850 M_{\odot} \mathrm{pc}^{-3}$. We note that two different curves are obtained although the same values for the fitting parameters were used. One can see that the density parameter $\rho_{\mathrm{s}}$ is high, and is the highest value found for any galaxy in our sample. With regard to the MOND modeling, both isotropic and anisotropic models are shown in the lower panel of Fig. 13. The fits of the inner part (based on $\gamma_{\text {in }}$ ) are shown in Fig. 13, as seen also in Table 7. As can be seen, all three anisotropic models based on $\beta_{\text {lit }}$, require some amount of DM in the inner part ("in"), out to $\sim 2 \operatorname{arcmin}\left(=3.5 R_{\mathrm{e}}\right)$ to fit the LOS observed velocity dispersion. The standard MOND model requires the highest mass-to-light ratio, $6.0<M / L_{B}<20.2$. In this case and also for other two tested MOND approaches, the upper limit is obviously higher than the values predicted by the tested SPS models by implying the existence of DM in the case of the anisotropic model based on $\beta_{\text {lit }}$. The simple MOND model provided a fit

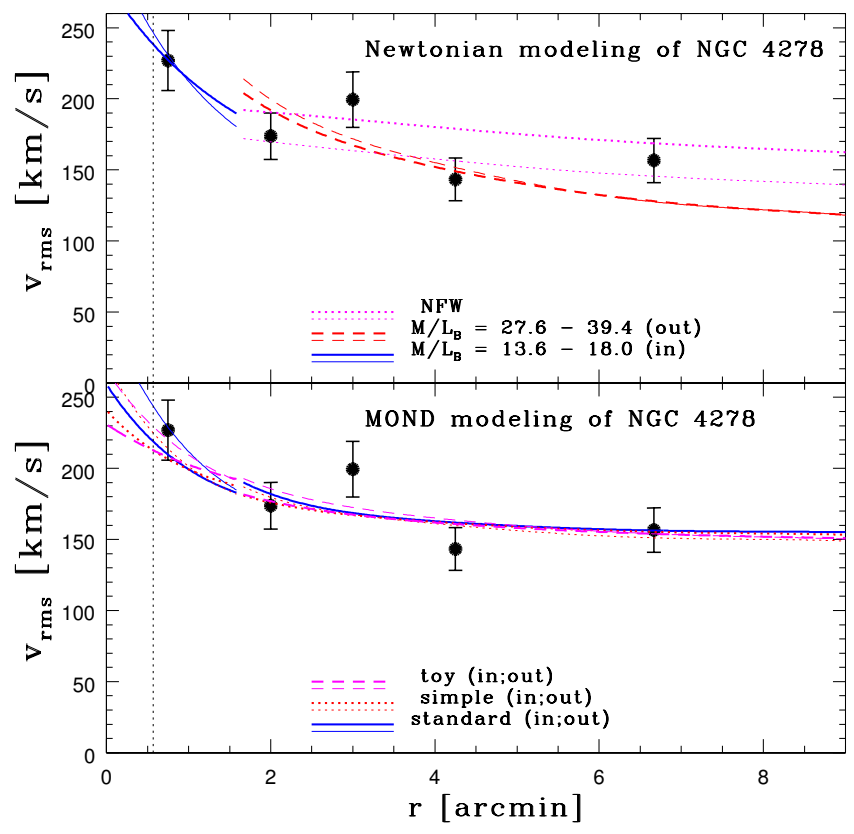

Fig. 13. Jeans Newtonian and MOND modeling of the projected velocity dispersion of NGC 4278 . The thick lines are for isotropic cases and the thin lines are for fits based on $\beta_{\text {lit }}$. Upper panel: fits of the projected velocity dispersion of NGC 4278 using the Newtonian approach for the inner ("in", solid blue lines) and outer ("out", dashed red lines) part. In the inner part, the thick line is for $M / L_{B}=13.6$, and the thin line is for $M / L_{B}=18.0$. In the outer part, the thick line is for $M / L_{B}=27.6$, and the thin line is for $M / L_{B}=39.4$. The NFW models with $M_{*} / L_{B}=9.5$, $r_{\mathrm{s}}=180 \operatorname{arcsec}$, and $\rho_{\mathrm{s}}=0.0850 M_{\odot} \mathrm{pc}^{-3}$ are shown with the thick (thin) dotted magenta lines. Lower panel: the galaxy is split into its inner ("in") and outer ("out"). The values of the mass-to-light ratio of the fits are: for the standard MOND model, $M / L_{B}=11.3$ (22.4) for the inner (outer) part for the isotropic case, and $M / L_{B}=17.2(28.0)$ for the inner (outer) part for the anisotropic case based on $\beta_{\text {lit }}$. For the simple MOND model, $M / L_{B}=9.6$ (16.2) for the inner (outer) part for the isotropic case and $M / L_{B}=13.4$ (23.6) for the inner (outer) part for the anisotropic case based on $\beta_{\text {lit }}$, and for the toy MOND model $M / L_{B}=7.8$ (12.4) for the inner (outer) part for the isotropic case and $M / L_{B}=11.6$ (19.4) for the inner (outer) part for the anisotropic case based on $\beta_{\text {lit }}$. In both panels, one effective radius is indicated by a vertical dotted line.

with $5.2<M / L_{B}<16.6$, and the lowest mass-to-light ratio (but still partially incompatible with the stellar matter only) was obtained in the case of the toy model, $2.6<M / L_{B}<14.6$ (see Tables 7 and 5). The values of the mass-to-light ratio in the inner region of NGC 4278 from the three MOND models for the transition from the MOND to Newtonian regimes are consistent with the estimates found for all tested SPS models, although there is a hint for the need of DM also there. In the outer region, all three tested MOND models certainly require $\mathrm{DM}$ to provide a fit beyond $4.2 R_{\mathrm{e}}$ and a set of fits in this region ("out") thus suggest the existence of additional, unseen matter there. The allowed intervals of the mass-to-light ratio in the outer part of NGC 4278 are: $14.6<M / L_{B}<31.00$ (standard MOND model), $10.8<M / L_{B}<26.8$ (simple MOND model), and $8.4<M / L_{B}<22.4$ (toy MOND model). The mass-followslight Newtonian models clearly indicate the need of DM in the outer parts of the galaxy (beyond $\sim 3 R_{\mathrm{e}}$ ).

Our estimate of the mass-to-light ratio in the outer region of NGC 4278 (using Newtonian mass-follows-light approach; see Table 6) agrees with the result of Bertola et al. (1993), who used a HI disk to infer the mass-to-light ratio $M / L_{B}=45$ (at $\sim 10 R_{\mathrm{e}}$ ). 


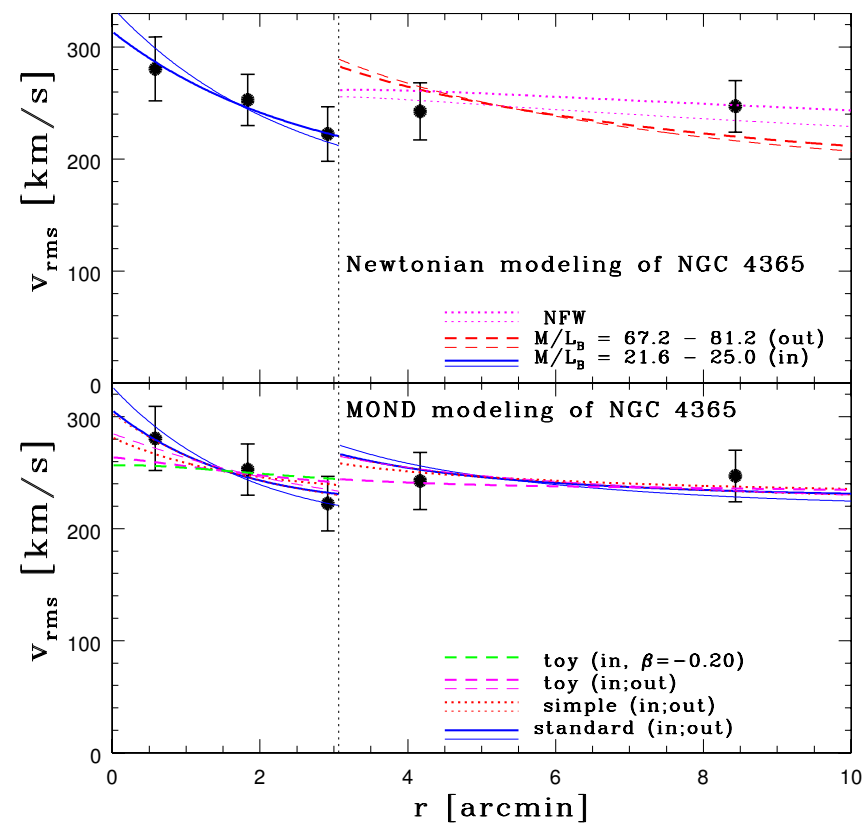

Fig. 14. Jeans Newtonian and MOND modeling of the projected velocity dispersion of NGC 4365 . The thick lines are for isotropic cases and the thin lines are for fits based on $\beta_{\text {lit. }}$ Upper panel: fits of the projected velocity dispersion of NGC 4365 using the Newtonian approach for the inner ("in", solid blue lines) and outer ("out", dashed red lines) part. In the inner part, the thick solid line is for $M / L_{B}=21.6$, and the thin solid line is for $M / L_{B}=25.0$. In the outer part, the thick dashed line is for $M / L_{B}=67.2$, and the thin dashed line is for $M / L_{B}=81.2$. The NFW models with $M_{*} / L_{B}=10.0, r_{\mathrm{s}}=250 \operatorname{arcsec}$ and $\rho_{\mathrm{s}}=0.0500(0.0550) M_{\odot} \mathrm{pc}^{-3}$ are shown with the thick (thin) dotted magenta lines. Lower panel: the galaxy is split into its inner ("in") and outer ("out"). The values of the mass-to-light ratio of the fits are as follows: for the standard MOND model, $M / L_{B}=20.4$ (56.8) for the inner (outer) part for the isotropic case and $M / L_{B}=23.6$ (70.6) for the inner (outer) part for the anisotropic case based on $\beta_{\text {lit }}$. For the simple MOND model, $M / L_{B}=17.2$ (43.2) for the inner (outer) part for the isotropic case and $M / L_{B}=20.2$ (54.4) for the inner (outer) part for the anisotropic case based on $\beta_{\text {lit. }}$. For the toy MOND model, $M / L_{B}=13.4$ (32.8) for the inner (outer) part for the isotropic case and $M / L_{B}=16.0$ (41.4) for the inner (outer) part for the anisotropic case based on $\beta_{\text {lit }}$. The toy model for the tangentially anisotropic case ( $\beta=-0.20$ ) for which $M / L_{B}=9.8$ is shown with the green dashed curve in the interior part of NGC 4365. In both panels, one effective radius is indicated by a vertical dotted line.

\subsection{NGC 4365}

The object NGC 4365 is a massive E3 galaxy behind the Virgo cluster. The basic observational data on NGC 4365 are given in Table 4. The kinematics of GCs of NGC 4365 are given in Fig. 4 (left).

For the fits below, we use the values of $n_{*}=7.11$ and $R_{\mathrm{e}}^{\text {(fit) }}=184.22 \operatorname{arcsec}$ (Kormendy et al. 2009). We note the higher value of the effective radius used in this paper with respect to that found in P13 that is equal to 60 arcsec (equivalent to $\sim 6.78 \mathrm{kpc}$ ). Our adopted value is equivalent to $\sim 20.8 \mathrm{kpc}$. Once again, the fits were done in an inner and outer region, and two different values of the $\gamma$ parameter, $\gamma=0.84$ (inner region) and $\gamma=2.10$ (outer region), that characterize the radial surface density were used, see Fig. 7.

The case of NGC 4365 is an example of a galaxy with a high mass-to-light ratio value in its inner region, which is interior to $1 R_{\mathrm{e}}$. The Newtonian fits of NGC 4365 are presented in the upper panel of Fig. 14. One can see that the velocity dispersion of NGC 4365 can be fit with a constant mass-to-light ratio without anisotropies (with the value $M / L_{B}=21.6$ ) and the anisotropic case based on $\beta_{\text {lit }}$ (with $M / L_{B}=25.0$ ). From the values reported in Table 6 , the interval of the mass-to-light ratio that provides a successful fit for the inner part, interior to $1 R_{\mathrm{e}}$ is $14.2<M / L_{B}<27.6$, and the observations of the GCs in the inner region suggest that DM is required for all tested cases. For all SPS models, there is a need for DM. Beyond $\sim 1 R_{\mathrm{e}}$, the Newtonian mass-follows-light models require significant amount of DM (see Tables 6 and 5). The interval of the allowed mass-to-light ratio becomes $48.4<M / L_{B}<92.6$. (The two fits from the given range are shown in the upper panel of Fig. 14: $M / L_{B}=67.2$ and $M / L_{B}=81.2$, respectively.) The NFW models with a stellar mass-to-light ratio, $M_{*} / L_{B}=10.0$, and a DM halo characterized by $r_{\mathrm{s}}=250 \operatorname{arcsec}$ and $\rho_{\mathrm{s}}=$ $0.0500(0.0550) M_{\odot} \mathrm{pc}^{-3}$ can provide a successful fit to the LOS observed velocity dispersion.

All tested MOND models require DM throughout the whole galaxy; the only exception is the tangentially anisotropic toy model (Fig. 14, see also Table 7 for details), which is marginally consistent with the IP13 Salpeter SPS model in the inner parts of NGC 4365, which is interior to $1 R_{\mathrm{e}}$. In the lower panel of Fig. 14, the three MOND models for the transition from the MOND to Newtonian regimes based on $\gamma_{\text {out }}$ are shown and are valid beyond $\sim 1 R_{\mathrm{e}}$. The standard MOND model requires the highest massto-light ratio, $39.0<M / L_{B}<81.6$; the simple MOND model provided a fit with $28.4<M / L_{B}<64.6$, and the lowest mass-tolight ratio (but still inconsistent with the stellar mass only) was obtained in the case of the toy model, $22.2<M / L_{B}<49.2$ (see Tables 7 and 5). None of the three MOND models for the transition from the MOND to Newtonian regimes can provide a fit beyond $\sim 1 R_{\mathrm{e}}$ without $\mathrm{DM}$, and a set of fits in this outer region ("out") indicates the existence of additional, unseen matter there.

Both mass-follows-light and MOND models imply the rise of the mass-to-light ratio and, thus, the existence of DM in the galaxy NGC 4365.

\subsection{NGC 4486}

The object NGC 4486 (=M87) is a massive giant E0 galaxy and is a member of the Virgo cluster found at its very center. The basic observational data on NGC 4486 are given in Table 4 . The kinematics of GCs of NGC 4486 are given in Fig. 4 (right).

The kinematics of GCs includes both "new" and "old" data, where the year 2003 was taken as the boundary (see Strader et al. 2011). The especially problematic region is $5<r<10$ arcmin where we see a significant increase in the velocity dispersion: in this region one finds 97 "old" out of 183 GCs (as noted by Strader et al., the "new" data set "has a sparse coverage at $R \sim 6.7$ arcmin $(\sim 32 \mathrm{kpc})$ where many of the old extreme velocities were"). Given the problem of reliability of the "old" data (Strader et al. 2011) and possible contamination in a aforementioned region, we did not attempt to model those discrepant observed points at this stage. More observations are certainly required to better establish the GCs kinematics between 5 and 10 arcmin. The outer regions beyond $\sim 10$ arcmin do not suffer from this problem, since all the observed GCs belong to the "new" group.

For the fits below, we use the values of $n_{*}=11.84$ and $R_{\mathrm{e}}^{(\mathrm{fit})}=$ 703.91 arcsec (Kormendy et al. 2009). We note that again the value of the effective radius used in this paper is much higher than that found in P13 that is equal to $81 \operatorname{arcsec}(=7.76 \mathrm{kpc})$; our adopted value is equivalent to $\sim 58.7 \mathrm{kpc}$. One can see that 


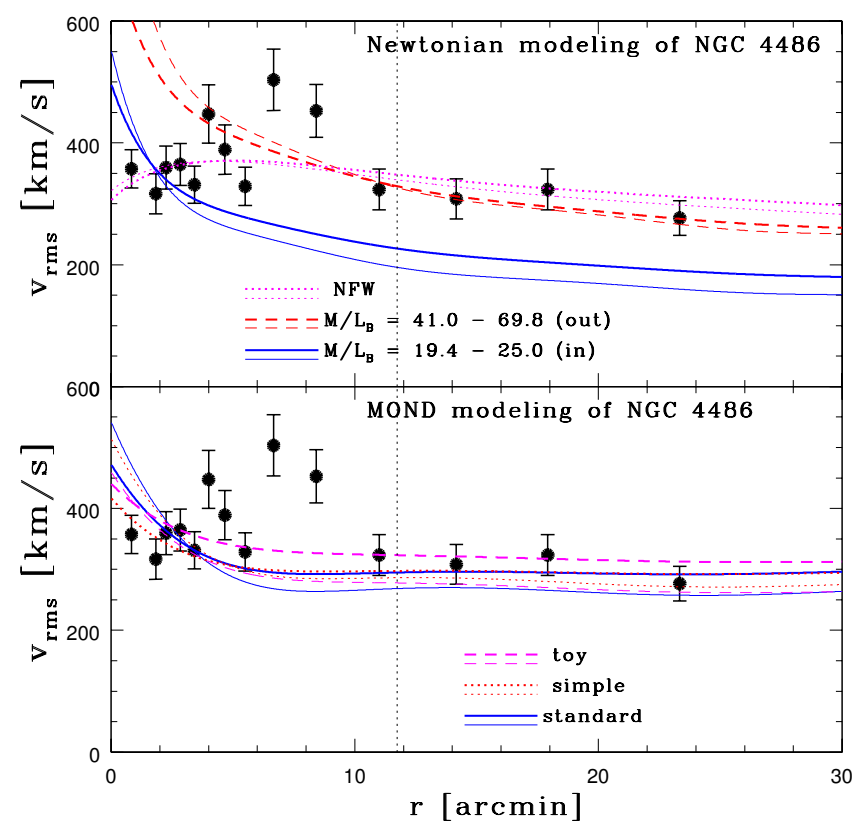

Fig. 15. Jeans Newtonian and MOND modeling of the projected velocity dispersion of NGC 4486. The thick lines are for isotropic cases and the thin lines are for fits based on $\beta_{\text {lit }}$. Upper panel: fits of the projected velocity dispersion of NGC 4486 using the Newtonian approach for the inner ("in", solid blue lines) and outer ("out", dashed red lines) part. In the inner part, the thick line is for $M / L_{B}=19.4$, and the thin line is for $M / L_{B}=25.0$. In the outer part, the thick line is for $M / L_{B}=41.0$ and the thin line is for $M / L_{B}=69.8$. The NFW models with $M_{*} / L_{B}=6.5(7.5), r_{\mathrm{s}}=300(350)$ arcsec and $\rho_{\mathrm{s}}=0.0700(0.0750) M_{\odot} \mathrm{pc}^{-3}$ are shown with the thick (thin) dotted magenta lines. Lower panel: standard MOND model is presented using $M / L_{B}=20.0$ (28.8) for the isotropic (anisotropic case based on $\beta_{\text {lit }}$ ); the simple MOND model is presented using $M / L_{B}=15.4$ (25.8) for the isotropic (anisotropic case based on $\beta_{\text {lit }}$ ), and the toy MOND model is presented using $M / L_{B}=15.4$ (18.4) for the isotropic (anisotropic case based on $\beta_{\text {lit }}$ ). In both panels, one effective radius is indicated by a vertical dotted line.

the values of both parameters are very high (they are in both cases the highest in the sample) and they are obtained using a composite profile of the surface brightness of NGC 4486 taking observations from the available literature into account.

As shown below, the case of NGC 4486 is the only example of the galaxy for which the mass of the inner region (interior to $\sim 0.35 R_{\mathrm{e}}$, equivalent to $\sim 15 \mathrm{kpc}$ ) is either dominated by the dark component (for the isotropic or radially anisotropic case) or its contribution is appreciable (for the tangentially anisotropic case). In Fig. 15, one may see that the velocity dispersion of NGC 4486 can be fit with a constant mass-to-light ratio without anisotropies (the value $M / L_{B}=19.4$ is plotted) and the anisotropic case based on $\beta_{\text {lit }}$ with $M / L_{B}=25.0$ is plotted. Taking into account the values found in Table 6 , the interval of the values that provide a successful fit for the inner part, interior to $\sim 0.35 R_{\mathrm{e}}$, is $12.6<M / L_{B}<27.0$, and the observations of the GCs suggest that DM is required in the inner region of NGC 4486 (for BC, PEGASE and IP13 Salpeter models, see Table 5). Beyond $\sim 0.35 R_{\mathrm{e}}$, the observed values of the velocity dispersion suggest that the dark component becomes more important. The allowed interval of the mass-to-light ratio becomes $25.6<M / L_{B}<72.8$. The NFW models, which are isotropic (anisotropic case based on $\beta_{\text {lit }}$ ) with a stellar mass-tolight ratio, $M_{*} / L_{B}=6.5(7.5)$, with a DM halo characterized by $r_{\mathrm{s}}=300(350)$ arcsec and $\rho_{\mathrm{s}}=0.0700(0.0750) M_{\odot} \mathrm{pc}^{-3}$ provide a successful fit to the LOS observed velocity dispersion throughout the whole galaxy. In the lower panel of Fig. 15, the three MOND models for the transition from the MOND to Newtonian regimes are plotted. As in all the cases studied above, the standard MOND model requires the highest massto-light ratio, $12.4<M / L_{B}<33.6$ (the values $M / L_{B}=20.0$ and $M / L_{B}=28.8$ are shown with the solid lines); obviously, DM is necessary for the standard MOND model (see Tables 6 and 5). The fits based on the simple MOND model for which $10.6<M / L_{B}<27.4$ provide the similar conclusion, as in the previous case again by indicating the need for the additional, dark component. The toy MOND model although predicts the lower mass-to-light ratio, $9.6<M / L_{B}<19.6$, suggests that DM is necessary in NGC 4486 because the obtained values of the mass-to-light ratios are always higher than the values predicted by the SPS models. This is the only galaxy in our sample for which the MOND models without DM cannot provide a fit in its inner regions (interior to $\sim 15 \mathrm{kpc}$ ).

Strader et al. (2011) showed that the surface brightness profile of red GCs follows closely the stellar light distribution of NGC 4486 (Kormendy et al. 2009) and that the deviations occur in the central few kiloparsecs (interior to $\sim 1.6$ arcmin), where the GCs have a flat core-like distribution and also in the very outer halo (beyond $\sim 20 \mathrm{arcmin}$ ). If one wants to assess the effects of the incompleteness (see Sect. 2), one can use the photometric slope based on the galaxy light distribution, which avoids the radial incompleteness issue to establish the mass-tolight ratio in the inner part of NGC 4486. At values interior to $\sim 1.6$ arcmin, the stellar photometric slope is somewhat steeper $(\gamma=0.79 \pm 0.02)$. The best-fit value of the mass-to-light ratio increases, and, for example, for the Newtonian mass-followslight isotropic case, it will become $M / L_{B}=21.0$, which is an increase of approximately 8 percent with respect to the result obtained using the slope based on the total sample of GCs $\left(M / L_{B}=19.4\right.$, see Fig. 15). Thus, the possible incompleteness issue would result in an increase of the total mass-to-light ratio in the inner parts, which leads to the conclusion that the contribution of the invisible dark, component is even larger there. The conclusions also reached in the case of the MOND models remain: NGC 4486 is the only galaxy for which no MOND model without the contribution of DM could be found. Strader et al. (2011) provide their estimates of the mass-to-light ratio of NGC 4486. They found: a) $M / L_{B}=20 \pm 9$ (at $\sim 2.2$ arcmin in the inner parts) and b) $M / L_{B}=46 \pm 15$ (at $\sim 16.8$ arcmin in the outer parts). Both values are consistent with our results (see Fig. 15 and Table 6).

The mass-to-light ratio based on GCs interior to $\sim 8.75$ arcmin of NGC 4486 obtained in D12, $M / L_{B}=43 \pm 4$, is consistent with our value obtained by using Newtonian mass-follows-light approach for the isotropic case: $41.0 \pm 2.6$ (see Table 6).

\subsection{NGC 4494}

The object NGC 4494 is an E1-E2 elliptical galaxy in the outer regions of the Virgo cluster. The basic observational data on NGC 4494 are given in Table 4. The kinematics of GCs of NGC 4494 are given in Fig. 5.

The values of $n_{*}=3.4$ and $R_{\mathrm{e}}^{\text {(fit) }}=45 \operatorname{arcsec}$ (Krajnović et al. 2013) are used in the Jeans fits below. As was done above when two values of the $\gamma$ parameter were used, the fits were determined in two regions: $\gamma=0.73$ (inner region) and steeper $\gamma=2.18$ (outer region), as seen in Fig. 7. Using Newtonian mass-follows-light models for NGC 4494, we can see that one cannot fit the LOS observed velocity dispersion with a single 


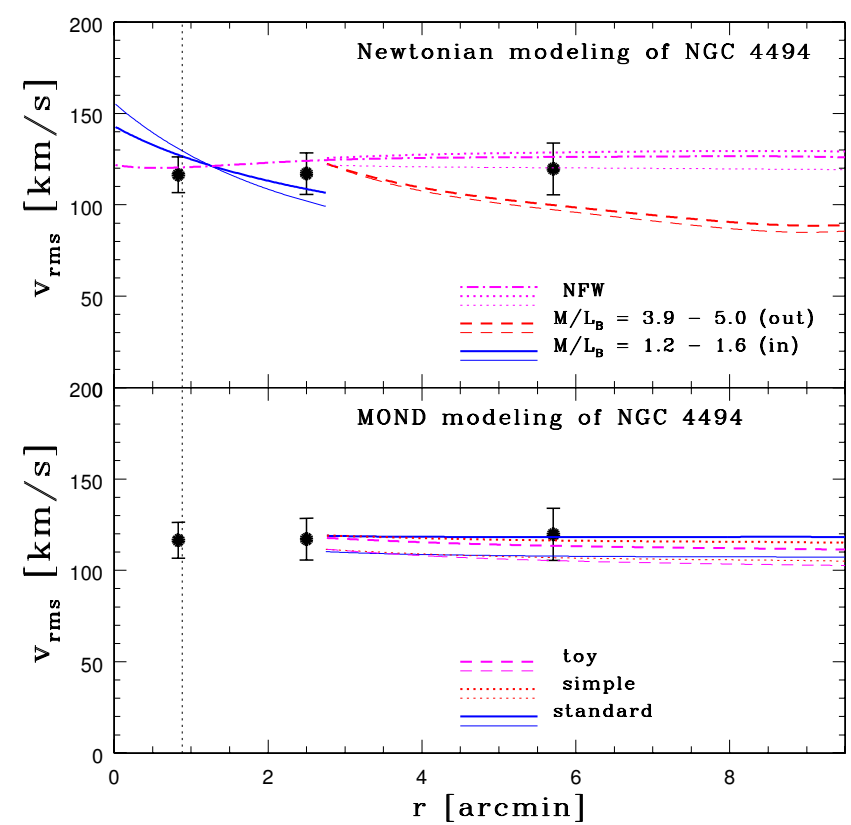

Fig. 16. Jeans Newtonian and MOND modeling of the projected velocity dispersion of NGC 4494. The thick lines are for isotropic cases, and the thin lines are for fits based on $\beta_{\text {lit }}$. Upper panel: fits of the projected velocity dispersion of NGC 4494 using the Newtonian approach for the inner ("in", solid blue lines) and outer ("out", dashed red lines) part. In the inner part, the thick line is for $M / L_{B}=1.2$, and the thin line is for $M / L_{B}=1.6$. In the outer part, the thick line is for $M / L_{B}=3.9$ and the thin line is for $M / L_{B}=5.0$. The NFW models with $M_{*} / L_{B}=1.5$ (2.2), $r_{\mathrm{s}}=600(650)$ arcsec, and $\rho_{\mathrm{s}}=0.0040(0.0035) M_{\odot} \mathrm{pc}^{-3}$ are shown with the thick (thin) dotted magenta lines. The thick dot-dashed magenta line is for the isotropic NFW model for which $M / L_{B}=1.2$, $r_{\mathrm{s}}=550$ arcsec, and $\rho_{\mathrm{s}}=0.0035 M_{\odot} \mathrm{pc}^{-3}$. Lower panel: standard MOND model is presented using $M / L_{B}=2.2$ (2.4) for the isotropic (anisotropic case based on $\beta_{\text {lit }}$ ). The simple MOND model is presented using $M / L_{B}=1.6(1.8)$ for the isotropic (anisotropic case based on $\beta_{\text {lit }}$ ), and the toy MOND model is presented using $M / L_{B}=1.2$ (1.4) for the isotropic (anisotropic case based on $\beta_{\text {lit }}$ ). In both panels, one effective radius is indicated by a vertical dotted line.

mass-to-light ratio throughout the whole galaxy (see the upper panel of Fig. 16). In the inner part $\left(\sim 3 R_{\mathrm{e}}\right)$, the mass-to-light ratio is in the interval $0.6<M / L_{B}<1.8$, which suggests an unusually low mass-to-light ratio of this galaxy. Beyond $\sim 3 R_{\mathrm{e}}$, in the outer region of NGC 4494, an increase in the mass-to-light ratio is required to fit the outermost point at $\sim 6 R_{\mathrm{e}}$. The allowed interval for the mass-to-light ratio then becomes $2.6<M / L_{B}<5.7$, which again indicates low mass-to-light ratio of NGC 4494, even in the outer parts (see Tables 6 and 5). To fit the outermost point, one requires a higher mass-to-light ratio, $M / L_{B} \sim 7.0$. Here, the IP13 Kroupa models provide the best agreement with the estimates obtained from the Jeans models. We note that all stellar population models suggest that NGC 4494 (with NGC 3115) has the lowest mass-to-light ratio of all ten galaxies in the sample, and this is indeed confirmed with our Jeans modeling. The two NFW models presented in Fig. 16 with a stellar mass-tolight ratio, $M_{*} / L_{B}=1.5(2.2)$, with a DM halo characterized by $r_{\mathrm{s}}=600(650) \operatorname{arcsec}$ and $\rho_{\mathrm{s}}=0.0040(0.0035) M_{\odot} \mathrm{pc}^{-3}$ can provide a successful fit to the LOS observed velocity dispersion. We note that the obtained values of $\rho_{\mathrm{s}}$ are the lowest for any galaxy in the sample. Again, as in the case of NGC 1407, we checked whether the NFW models can provide a fit throughout the whole galaxy, and we present the best fit based on a single parameter $\alpha$ valid in both the inner and the outer regions, $\alpha=2.57$. The best fit $\left(\chi^{2}=0.68\right)$ for the isotropic case with $M_{*} / L_{B}=1.2, r_{\mathrm{s}}=550 \operatorname{arcsec}$, and $\rho_{\mathrm{s}}=0.0035 M_{\odot} \mathrm{pc}^{-3}$ is plotted with the thick dot-dashed line in Fig. 16. Here, the quality of the fit is comparable to that found for the fit in the outer region, and we conclude that the NFW models (also the two anisotropic case not shown here) can provide a fit for NGC 4494. All three MOND models for the transition from the MOND to Newtonian regimes can provide a fit to the LOS observed velocity dispersion consistent with the lack of DM throughout the whole galaxy. As in the Newtonian case, the values for the fit of mass-to-light ratios are very low: the standard MOND model as usual requires the highest mass-to-light ratio, $1.4<M / L_{B}<2.6$; the simple MOND model provided a fit with $1.0<M / L_{B}<2.2$, and the lowest mass-to-light ratio was obtained in the case of the toy model, $0.8<M / L_{B}<1.6$ (see Tables 7 and 5). The overall conclusion regarding NGC 4494 is that DM is required out to $\sim 6 R_{\mathrm{e}}$ unless we assume that there is an increase of the stellar mass-to-light ratio by a factor of $\sim 4$ with radius between the innermost and outermost radii in this galaxy; only a detailed observational study of the stellar populations in NGC 4494 that addresses possible internal stellar mass-to-light ratio gradients will resolve this problem. Because there is a hint of increasing mass-to-light ratio at the outermost radii in the Newtonian massfollows-light model, a more detailed study of stellar population is certainly required to clarify the contribution (or lack) of the dark component.

The mass-to-light ratio of NGC 4494 is also the lowest in the sample of 15 galaxies found in D12 (using PNe): they found that interior to $5 R_{\mathrm{e}}, M / L_{B}=5 \pm 1$, which is consistent with our result (for the outer part of the galaxy, see Table 6). Napolitano et al. (2009) studied DM in NGC 4494 using a sample of $255 \mathrm{PNe}$ out to $\sim 7 R_{\mathrm{e}}$ : they found that at $5 R_{\mathrm{e}}$, the dynamical mass-to-light ratio is $6<M / L_{B}<10$, which also agrees with our result. We also note that their NFW halo parameters $r_{\mathrm{s}}=410 \pm 30 \operatorname{arcsec}$ and $\rho_{\mathrm{s}}=0.0019 \pm 0.0006 M_{\odot} \mathrm{pc}^{-3}$ agree with our result (see Table 6). NGC 4494 is another galaxy analyzed by Milgrom \& Sanders (2003), who used the PNe observations of Romanowsky et al. (2003) and showed that the standard MOND formula for $M / L_{B}=5.4$ (the Hernquist 1990 model of the light distribution was applied) can describe the LOS observed velocity dispersion of NGC 4494 without the need of DM. More recently, Morganti et al. (2013) analyzed NGC 4494 using the NMAGIC code applied to the observations of approximately $260 \mathrm{PNe}$, which extend out to $\sim 7 R_{\mathrm{e}}$ and found that their best-fitting models are described with $M / L_{B}=3.71 \pm 0.15$ with the $\mathrm{DM}$ fraction interior to $\sim 5 R_{\mathrm{e}}$ equal to $0.6 \pm 0.1$.

\subsection{NGC 5846}

The object NGC 5846 is a massive E0 galaxy and is a member of a group. The basic observational data on NGC 5846 are given in Table 4. The kinematics of GCs of NGC 5846 are given in Fig. 5.

For the fits below, we use the values of $n_{*}=3.9$ and $R_{\mathrm{e}}^{(\mathrm{fit})}=67.4 \operatorname{arcsec}$ (Krajnović et al. 2013). As shown, the fits were performed in an inner and outer region, and again, two different values of the $\gamma$ parameter that characterize the radial surface density were used: $\gamma=0.81$ (inner region, interior to $\sim 3 R_{\mathrm{e}}$ ) and steeper $\gamma=4.15$ (outer region, beyond $\sim 3 R_{\mathrm{e}}$ ), see Fig. 7. The case of the galaxy NGC 5846 is the only case where we modeled the LOS observed velocity dispersion using a Newtonian mass-follows-light approach in three separate regions because no mass-follows-light model could provide a fit 


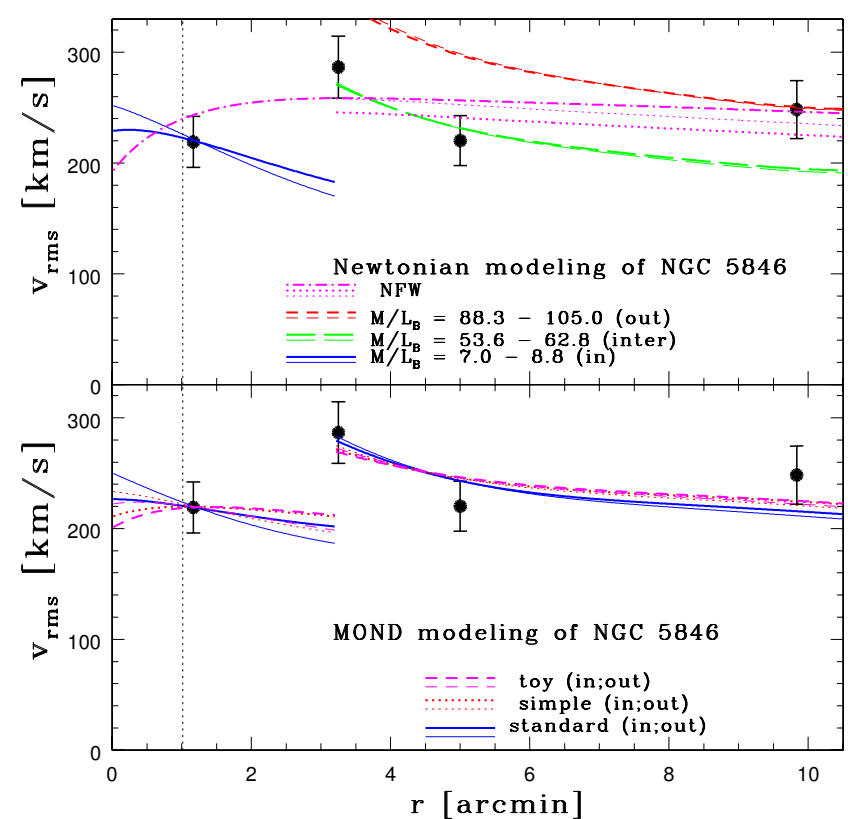

Fig. 17. Jeans Newtonian and MOND modeling of the projected velocity dispersion of NGC 5846. The thick lines are for isotropic cases and the thin lines are for fits based on $\beta_{\text {lit. }}$ Upper panel: isotropic Jeans modeling of the projected velocity dispersion of NGC 5846 using the Newtonian approach. For the Newtonian mass-follows-light approach, the three regions are as follows: inner region (interior to $2 R_{\mathrm{e}}$, solid blue lines), intermediate region (between $2 R_{\mathrm{e}}$ and $6 R_{\mathrm{e}}$, long dashed green lines), and outer region (beyond $6 R_{\mathrm{e}}$, short dashed red lines). The massto-light ratio in these regions are $M / L_{B}=7.0(8.8), 53.6(62.8)$ and 88.3 (105.0) for the thick (thin) lines. The NFW models with $M_{*} / L_{B}=$ $9.0(8.5), r_{\mathrm{s}}=300 \operatorname{arcsec}$, and $\rho_{\mathrm{s}}=0.0450(0.0600) M_{\odot} \mathrm{pc}^{-3}$ are shown with the thick (thin) dotted magenta lines. The thick dot-dashed magenta line is for the isotropic NFW model for which $M / L_{B}=7.0, r_{\mathrm{s}}=$ $350 \operatorname{arcsec}$, and $\rho_{\mathrm{s}}=0.0200 \mathrm{M}_{\odot} \mathrm{pc}^{-3}$. Lower panel: isotropic MOND models of the projected velocity dispersion of NGC 5846 for three interpolation functions $\mu(r)$ split into two regions. The inner region is interior to $2 R_{\mathrm{e}}$, and the outer region is beyond $2 R_{\mathrm{e}}$. The standard MOND model is presented with the solid blue lines for $M / L_{B}=6.7$ (8.5) (inner region) and $M / L_{B}=55.2$ (66.0) (outer region); the simple MOND model is presented with the dotted red lines for $M / L_{B}=5.7$ (7.3) (inner region) and $M / L_{B}=44.8$ (54.2) (outer region), and the toy MOND model is presented with the dashed red lines for $M / L_{B}=4.4$ (5.7) (inner region) and $M / L_{B}=34.2$ (41.6) (outer region). In both panels, one effective radius is indicated by a vertical dotted line.

throughout the whole galaxy. The Newtonian fits of NGC 5846 are presented in Fig. 17. The inner region interior to $\sim 2 R_{\mathrm{e}}$ can be fit with $4.2<M / L_{B}<10.6$, and this is compatible with all the tested SPS models, except the CvD12 Kroupa MW model (see Table 5), implying either the lack of DM or a low amount there; the cases for which $M / L_{B}=7.0$ (8.8) are shown on the graph. Between $\sim 2 R_{\mathrm{e}}$ and $\sim 6 R_{\mathrm{e}}$, the mass-to-light ratio increases, and the interval for which the fit can be obtained is $42.4<M / L_{B}<71.6$. This result implies the increasing fraction of DM in this region; the cases for which $M / L_{B}=53.6$ (62.8) are also shown. Finally, in the outermost region, the mass-to-light ratio continues to increase between $\sim 6 R_{\mathrm{e}}$ and $\sim 10 R_{\mathrm{e}}$ and the values $64.2<M / L_{B}<127.4$ provide a fit to the LOS observed velocity dispersion. The cases for which $M / L_{B}=88.3$ (105.0) are shown with the thick (thin) short dashed line. With NGC 4365 (for which $36.6<M / L_{B}<74.0$ in the outer parts, was established above), this is the highest value of the obtained mass-to-light ratio in the mass-follows-light approximation for any galaxy in our sample. The NFW models with a stellar mass-to-light ratio, $M_{*} / L_{B}=9.0(8.5)$, with a DM halo characterized by $r_{\mathrm{s}}=300 \operatorname{arcsec}$ and $\rho_{\mathrm{s}}=0.0450(0.0600) M_{\odot} \mathrm{pc}^{-3}$ also provide a successful fit to the LOS observed velocity dispersion. As in the cases of NGC 1407 and NGC 4494 above, we again checked whether the NFW models can provide a fit throughout the whole galaxy, and we present the best fit based on a single parameter $\alpha$ valid in both the inner and the outer regions, $\alpha=2.89$, and the best fit, $\chi^{2}=3.84$, for the isotropic case with $M_{*} / L_{B}=7.0, r_{\mathrm{s}}=350 \operatorname{arcsec}$ and $\rho_{\mathrm{s}}=0.0200 M_{\odot} \mathrm{pc}^{-3}$ is plotted in Fig. 17. The quality of the fit is comparable to that found for the fit in the outer region, and we conclude that the NFW models (also the two anisotropic case not shown here) can provide a fit for NGC 5846. In the lower panel of Fig. 17, the three isotropic MOND models are plotted for two separate regions: the inner region is again interior to $2 R_{\mathrm{e}}$ but the exterior region now extends from $2 R_{\mathrm{e}}$ to $\sim 10 R_{\mathrm{e}}$. The reason for this division is that none of the tested MOND models could provide a successful fit throughout the whole galaxy. As can be seen from Fig. 17 and Tables 7 and 5, the values of the mass-tolight in the inner region are consistent with the no dark-matter hypothesis: the allowed interval of the mass-to-light ratio in this region is $4.0<M / L_{B}<10.4$ (for the standard MOND model), $3.0<M / L_{B}<9.0$ (for the simple MOND model) and $2.5<M / L_{B}<7.1$ (for the toy MOND model). The estimates of the mass-to-light ratio in the inner region of NGC $5846 \mathrm{ob}-$ tained using the three MOND models for the transition from the MOND to Newtonian regimes are consistent with all tested SPS models, except the CvD12 Kroupa models. The fits beyond $\sim 2 R_{\mathrm{e}}$ cannot be obtained without DM for any of the MOND models: in the case of the standard MOND models, the allowed interval is $42.6<M / L_{B}<74.6$; for the simple MOND model, the allowed interval is $34.6<M / L_{B}<62.2$. In the case of the toy MOND model, the range becomes $26.4<M / L_{B}<47.4$. The result that there is an increase in the value of the fixed mass-to-light ratio for all MOND models means that using the MOND approach with the fixed mass-to-light ratio as the only free parameter is not sufficient for explaining the LOS observed velocity dispersion of NGC 5846. As can be seen from Fig. 17, the mass-to-light ratio increases significantly beyond $\sim 2 R_{\mathrm{e}}$. Only NFW modeling could provide a successful fit throughout the whole galaxy. Again, both mass-follows-light and MOND models imply the rise of the mass-to-light ratio and, thus, the existence of DM in the galaxy NGC 5846.

The case of galaxy NGC 5846 is the only case, where we encounter the discrepancy between our result for the mass-tolight ratio and that found in D12. As given above, for a region between $\sim 2 R_{\mathrm{e}}$ and $\sim 6 R_{\mathrm{e}}$, we obtain $43.2<M / L_{B}<68.4$, using a Newtonian mass-follows-light approach, whereas D12 quote the value of $M / L_{B}=28 \pm 6$. We note that both allowed ranges of the mass-to-light ratio are indicative of the presence of DM in the outer parts of NGC 5846. The reason for this discrepancy most probably lies in the result that we fit the observed data point in our Jeans models at $\sim 3.25 \operatorname{arcmin}\left(=3.20 R_{\mathrm{e}}\right)$ for which the LOS observed velocity dispersion reaches its maximum $\left(\sim 287 \mathrm{~km} \mathrm{~s}^{-1}\right)$. The result for the mass-to-light ratio found in D12 was obtained using a sample of PNe from Coccato et al. (2009): in Fig. 7 of Coccato et al., one can see that the velocity dispersion of PNe does not show an increase and is below $200 \mathrm{~km} \mathrm{~s}^{-1}$. Further studies and detailed comparison between the two tracers will clarify this issue. However, as shown above, the observations indicate that the kinematics of NGC 5846 in its outer regions is incompatible with the existence of the stellar population only; it is important to stress that this conclusion is valid for both Newtonian and MOND approaches. 
The galaxy NGC 5846 was recently subject of the study of Napolitano et al. (2014), who used the same SLUGGS sample of GCs. They attempted to constrain the IMF using a radially extended dataset of GCs with the determination of the NFW parameters (they did not address MOND), a procedure performed earlier in Samurović (2012) on the early-type galaxy NGC 4472 (where MOND was also tested). Napolitano et al. studied blue and red GC subpopulations separately and tested four models. Their standard NFW halo (without adiabatic compression) for the isotropic case ("iso-noAC" model), although providing lower value of the concentration parameter $\left(c_{\mathrm{vir}}=7_{-5}^{+6}\right)$, is because of large error bars consistent with our estimate of the same quantity $\left(c_{\mathrm{vir}}=14.99_{-3.43}^{+2.36}\right)$. The same is true for the estimates of the virial mass. They obtain a higher value of the virial mass, $M_{\mathrm{vir}}=2.14_{-1.16}^{+2.13} \times 10^{13} M_{\odot}$, whereas our estimate is $M_{\text {vir }}=6.56_{-355}^{+3.61} \times 10^{12} M_{\odot}$ (see Table 8$)$. The value of the best-fitting stellar mass-to-light ratio obtained by Napolitano et al. $\left(M_{*} / L_{B}=10.7\right.$, converted to the $B$-band, for the same "iso-noAC" model) is consistent with our value found for the isotropic NFW model, for which we find $M_{*} / L_{B}=9.0 \pm 2.0$ (see our Table 6).

\section{Discussion}

In Figs. 18 and 19, we plotted the $M / L_{B}$ profiles in units of $R / R_{\mathrm{e}}$. For simplicity, only two points are plotted: the inner point is always at $2 R_{\mathrm{e}}{ }^{6}$ and the outer point is for the estimate of the massto-light ratio in the outermost bin (see Table 3). We note that in four cases (NGC 1407, NGC 3377, NGC 4365, and NGC 4494) we plotted asymmetric error bars to accurately present the higher values of the established mass-to-light ratios in the outermost bins. In these two figures, one can clearly see which stellar mass models agree with the dynamical modeling at low radii where the stars are expected to dominate (Mamon \& Łokas 2005). One can also see that only one galaxy from the sample shows no increase in the mass-to-light ratio (NGC 2768 in Fig. 18).

To compare with the SPS models, we plotted several horizontal lines for each galaxy based on the models presented in Table 5. The lower limits of the mass-to-light ratios of the SPS models are determined (where available) with the CvD12 models with the Kroupa MW IMF and the IMF with variation, and the upper values of the mass-to-light ratios are determined with the IP13 Salpeter models, while the BC, PEGASE and IP13 models based on the Kroupa IMF are in between (or at the bottom when the CvD12 estimate is missing).

The only field galaxy in the sample (fast rotator NGC 3115) shows one of the sharpest increase of the mass-to-light ratio among the galaxies in the sample; it is also worth noting that this is the object for which there is a discrepancy of the mass-to-light ratio based on the dynamical modeling with respect to the tested SPS models in the inner region (the same situation is also encountered in the case of NGC 4486). The central cluster galaxy (NGC 4486) shows a similar mass-to-light profile as a group galaxy, NGC 4278 (also a cored boxy galaxy). The IP13 models with Salpeter IMF show best agreement with the modeled massto-light ratios in the inner regions of the early-type galaxies in our sample. The BC and PEGASE models with Salpeter IMF are in good agreement too, whereas the CvD12 models provide the values that are far too low. Apart from NGC 2768, for which we did not detect the increase of the mass-to-light ration in our Jeans models, the highest fraction of stars at the outermost radius

\footnotetext{
6 The two exceptions are NGC 4365 and NGC 4486 for which the inner points are at $0.65 R_{\mathrm{e}}$ and $0.23 R_{\mathrm{e}}$, respectively.
}

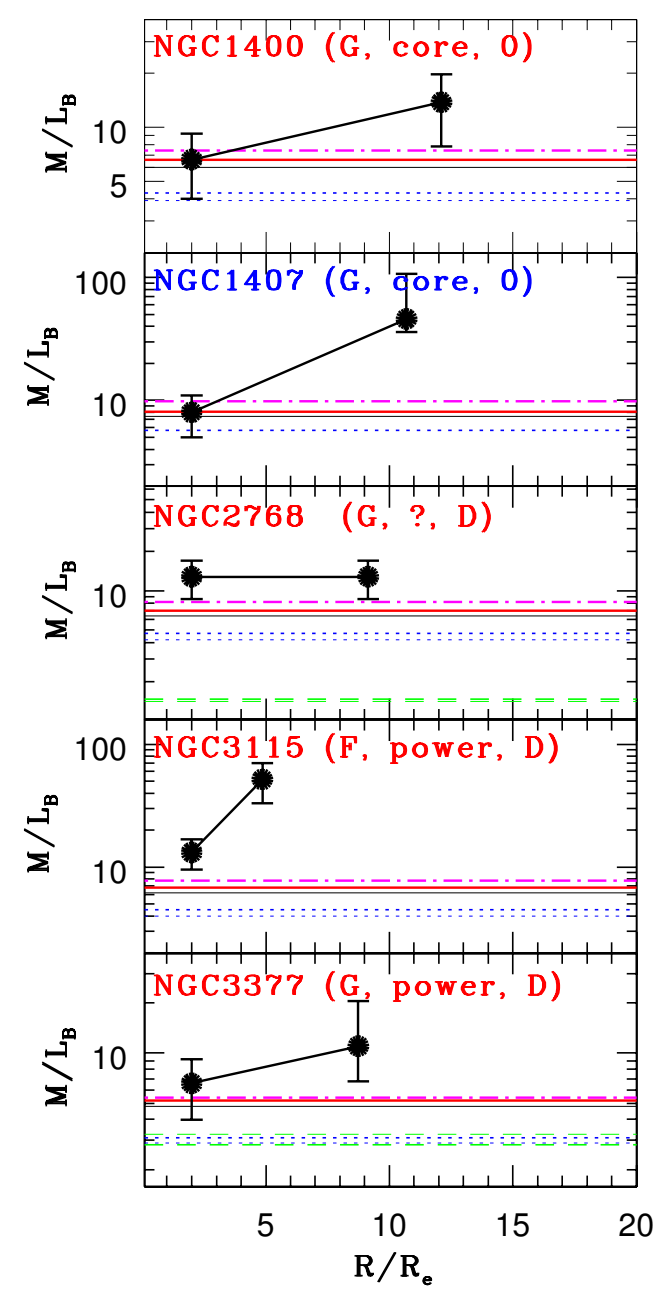

Fig. 18. Mass-to-light profiles of the galaxies in the sample based on the Newtonian models. For each object, two observational points are plotted: the inner one at $2 R_{\mathrm{e}}$ and the outer one at the outermost bin. For each galaxy, we indicate: the environment ("G" means that the galaxy belongs to a group, "F" is a field galaxy, and " $\mathrm{C}$ " means that the galaxy belongs to a cluster), profile type ("core" is for core, " $\cap$ " in Table 4 , "power" is for power low, "’" in Table 4, and "?" is unknown) and mean isotope shape (" $\mathrm{D}$ " indicates disky isophotes, " $\mathrm{B}$ " indicates boxy isophotes, and " 0 " indicates pure ellipses). The meaning of the horizontal lines is as follows: thin solid black lines are for the BC models; thick red solid lines are for the PEGASE models; dotted blue lines are for the exponential SFH IP13 models with the Kroupa IMF; thick dotted blue lines are for the IP13 disc galaxy model based on the Kroupa IMF; thick dot-dashed line is for the IP13 disk galaxy model based on the Salpeter IMF; thin green dashed lines are for the CvD12 models allowing for IMF variation; and thick green dashed lines are for the CvD12 models assuming a fixed MW (Kroupa) IMF. The names of the slow (fast) rotators are plotted using Roman characters (italic).

is for NGC 1400 and NGC 3377. In Fig. 19, one can also see the peculiar mass-to-light profile of NGC 4494: here, we encounter the situation that all the tested SPS models predict much higher estimates than the value obtained using the Jeans modeling (see discussion below).

The inner dynamical mass for each galaxy can be calculated using the following expression (Bertin et al. 2002):

$M_{\text {dyn }}=K_{\mathrm{v}} \frac{\sigma_{0}^{2} R_{\mathrm{e}}}{G}$,

where $K_{\mathrm{v}}=5$ (Cappellari et al. 2006) and $\sigma_{0}$ is the central velocity dispersion taken from the HyperLeda database. We note 
S. Samurović: Investigation of dark matter and MOND in early-type galaxies

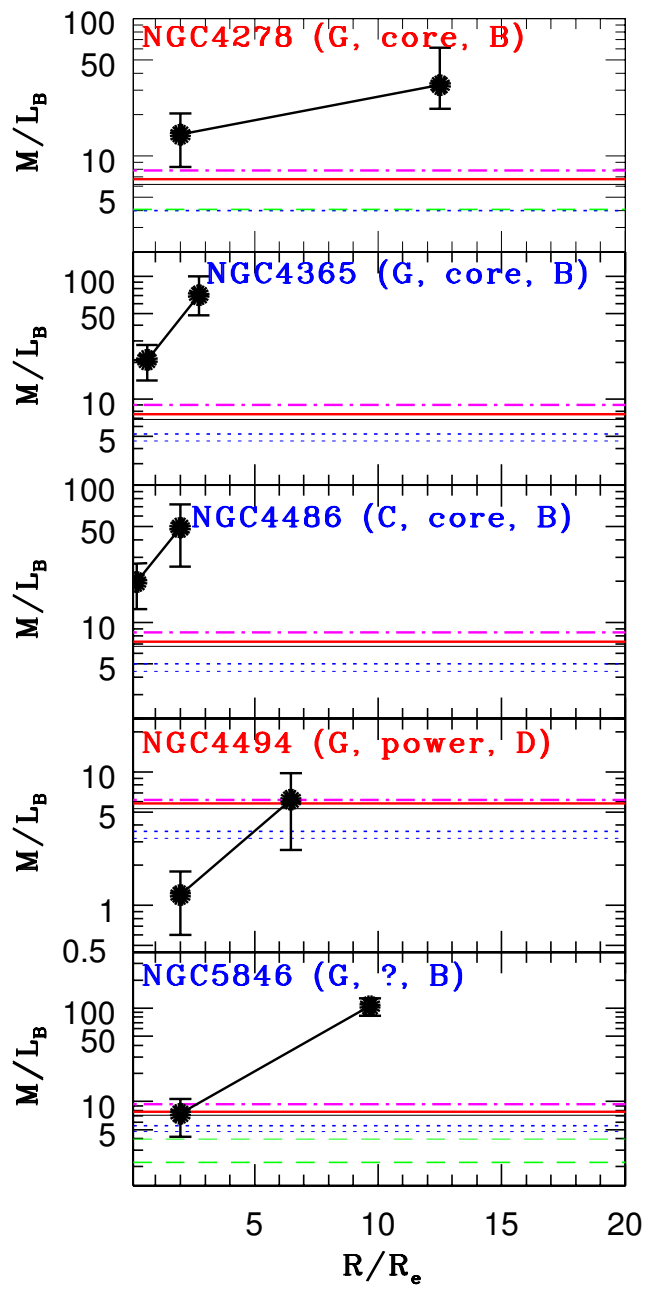

Fig. 19. Mass-to-light profiles of the galaxies in the sample based on the Newtonian models. The meaning of symbols is the same as in Fig. 18. We note that the values of the mass-to-light ratios for NGC 4365 and NGC 4486 appear close because of the values of their effective radii used in this paper (see text for details); the inner points for NGC 4365 and NGC 4486 are at $0.65 R_{\mathrm{e}}$ and $0.23 R_{\mathrm{e}}$, respectively.

that this equation is only correct for estimating the mass within the half-light radius: if the galaxy has NFW DM, then the constant, $K_{\mathrm{v}}$, is increased (see Courteau et al. 2014). The "dynamical mass" given above measures twice the total mass at the halfluminosity radius, where stars are expected to fully dominate the DM (see e.g. Mamon \& Łokas 2005); hence the total stellar mass of the galaxy. There is another problem with this estimate, and that is because the central supermassive black holes increase the values of inner velocity dispersions. When taken from catalogs (such as HyperLeda), they may come from observations made using different apertures with different point spread functions. Thus, we assumed that Eq. (17) is correct for the stellar masses. We added two sets of values to Figs. 20-23: dynamical mass-to-light ratios, $M_{\mathrm{dyn}} / L_{B}$, and the values of $M_{\mathrm{stars}} / L_{r}$ measured dynamically by Cappellari et al. (2013b, their Table 1) (after subtracting the DM component and after suitable color transformation), and $M_{\text {stars }} / L_{B}$ as asterisks. In such a way, one can compare the intervals of $M_{\text {stars }} / L_{B}$ of the different SPS models with $M_{\text {dyn }} / L_{B}$ and $M_{\text {stars }} / L_{B}$ from Cappellari et al. (2013b). From Figs. 20,21, one can quickly infer the contribution of DM, and from Figs. 22, 23, one can easily inspect the ability of

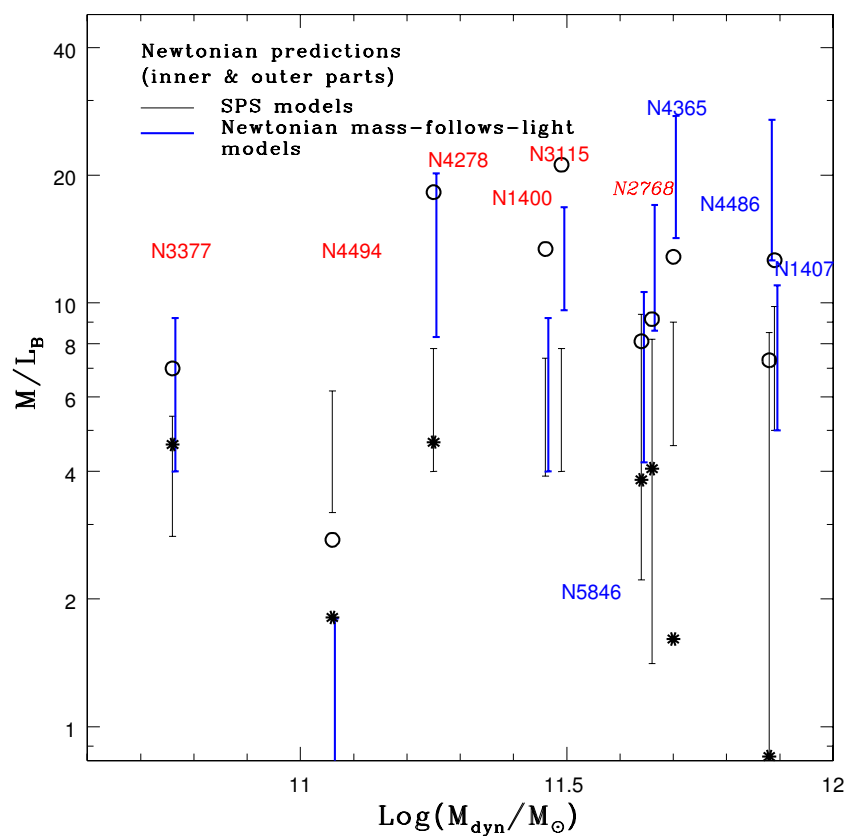

Fig. 20. Comparison of the Newtonian mass-follows-light models (thick solid lines, shifted for the sake of visibility) with the predictions of various SPS models (thin solid lines). For one galaxy for which the name was given with "W" in an exponent (NGC 2768), the comparison is made for its whole radial extent. For NGC 4365 and NGC 4486, the inner parts extend out to $1 R_{\mathrm{e}}$ and $0.35 R_{\mathrm{e}}$, respectively; for the remaining seven objects, this plot shows the comparison for their inner parts, interior to $\sim 3 R_{\mathrm{e}}$. Open circles and asterisks denote $M_{\mathrm{dyn}} / L_{B}$ (Eq. (17)) and $M_{\text {stars }} / L_{B}$ (dynamically measured by Cappellari et al. 2013b), respectively (see text for details). The names of the slow (fast) rotators are plotted using Roman characters (italic).

MOND to model the galaxies in the sample ${ }^{7}$. Figure 21 shows that none of the galaxies in our sample (except for the problematic NGC 4494) can be explained by the Newtonian model without DM. Figure 23 indicates that no MOND model can describe the kinematics of galaxies NGC 1407, NGC 3115, NGC 4278, NGC 4365, NGC 4486, nor NGC 5846 with stellar matter only.

One should note that when comparing Newtonian and MOND mass models, one should take into account that the velocity anisotropy in MOND might be different than for Newtonian dynamics: since massive galaxies in MOND are expected to lack DM halos, MOND galaxies are built as much by mergers as are the high mass galaxies in Newtonian dynamics (e.g. Cattaneo et al. 2011). When we compare Newtonian and MOND models it is important to stress that we compare $\chi_{\text {Newtonian-noDM,inner }}^{2}+\chi_{\text {Newtonian-noDM,outer }}^{2}, \chi_{\text {Newtonian-DM }}^{2}$ and $\chi_{\text {MOND-noDM }}^{2}$. Since mergers are also one way to put outer stars (and GCs) on very elongated orbits, galaxies built from Newtonian dynamics may have more radial orbits in their outer regions than MOND galaxies. On the other hand, mergers should be rare for galaxies of lesser mass (Cattaneo et al. 2011): in this case, the elliptical morphologies are reached by other processes than mergers, such as ram pressure stripping (Gunn \& Gott 1972) and galaxy harassment (see Mastropietro et al. 2005; Cattaneo et al. 2011). In the former, the disk is starved, and the galaxy should turn into a disky elliptical or S0. For the latter, the numerous minor non-merging encounters puff up the galaxy,

7 Note that three out of ten galaxies from our sample are missing in Cappellari et al. (2013b): NGC 1400, NGC 1407, and NGC 3115 (see Table 8). In such cases in Figs. 20-23, we plot only $M_{\text {dyn }} / L_{B}$ based on the values found in HyperLeda. 


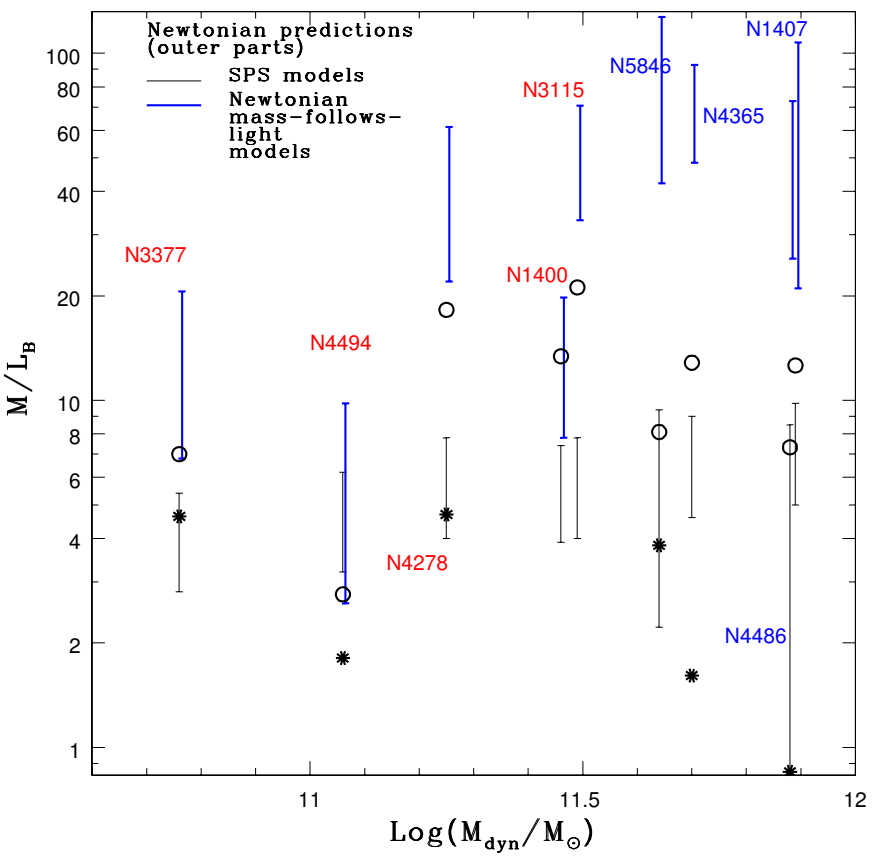

Fig. 21. Comparison of the Newtonian mass-follows-light models (thick solid lines, shifted for the sake of visibility) with the predictions of various SPS models (thin solid lines). In this plot, the comparison is made for the outer parts (exterior to $\sim 3 R_{\mathrm{e}}$, except for NGC 4365 and NGC 4486, for which the exterior parts are beyond $1 R_{\mathrm{e}}$ and $0.35 R_{\mathrm{e}}$, respectively) of the galaxies in the sample. The meaning of symbols is the same as in Fig. 20.

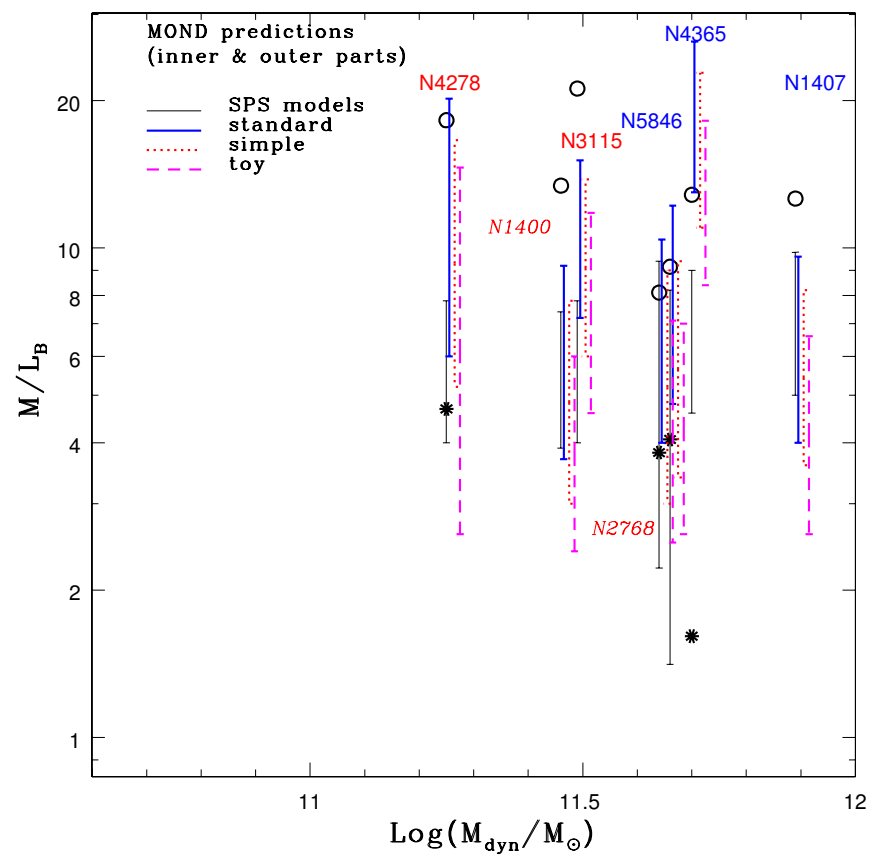

Fig. 22. Comparison of three different MOND models (standard MOND model is plotted with the thick solid lines, the simple MOND models is plotted with the thick dotted lines, and the toy MOND model is presented with the thick dashed lines; all models were shifted to the right for the sake of visibility) with the predictions of various SPS models (thin solid lines). For two galaxies for which the names were given in italics (NGC 1400 and NGC 2768), the comparison is made for their whole radial extent. For NGC 4365 and NGC 4486, the inner parts extend out to $1 R_{\mathrm{e}}$ and $0.35 R_{\mathrm{e}}$, respectively; for the remaining five objects, this plot shows the comparison for their inner parts, interior to $\sim 3 R_{\mathrm{e}}$. The meaning of symbols is the same as in Fig. 20.

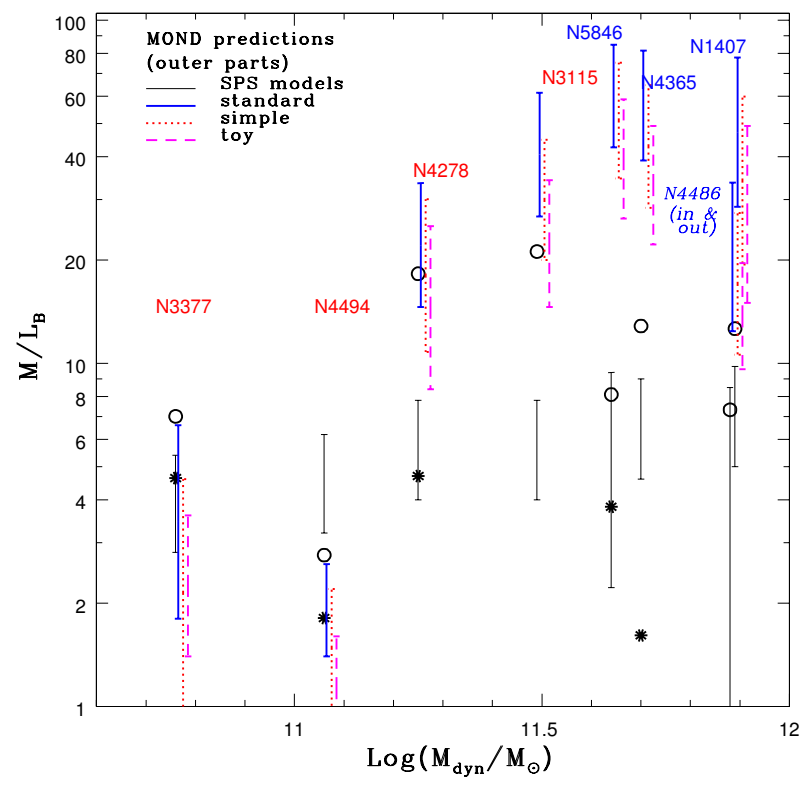

Fig. 23. Same as in Fig. 22 but the comparison for seven galaxies is made for their outer parts (exterior to $\sim 3 R_{\mathrm{e}}$ ). For NGC 4365 the exterior part is beyond $1 R_{\mathrm{e}}$, and for NGC 4486 , the comparison is made for both inner and outer regions. We note the upper value of the mass-to-light ratio is here $M / L_{B}=100$ (in Fig. 21 the upper value is $M / L_{B}=130$ ). The meaning of symbols is the same as in Fig. 20.

tidally destroying the outer disk. If the galaxies of the sample are in poor environments, ram pressure stripping is ineffective, while harassment is reduced. The environment of the galaxies from our sample is indicated in Table 4, and one can see that eight objects belong to groups, one galaxy (NGC 3115, the only So galaxy in the sample) is classified as a field galaxy and one galaxy (NGC 4486) is the central galaxy of the Virgo cluster. For the field galaxy, NGC 3115, we find the largest value of the concentration index (see Table 8), whereas the galaxies belonging to groups show a variety of values from small (5.38 for NGC 1400) to high (14.99 for NGC 5846), and NGC 4486 has a high value of the concentration parameter (16.77).

The Newtonian models with NFW DM provide better fits than the Newtonian models without DM for all galaxies (see Table 6): the only exception being NGC 3115 (for NGC 4486, both $\chi^{2}$ values are high because of the peculiar kinematics of this galaxy). The same is also true when one compares the quality of the fits obtained using NFW models and MOND: Only for NGC 3377, the quality of the MOND models is better, but the innermost point in that case could not be fit. For the Newtonian mass-following-light models, isotropic and mildly tangential anisotropic models provide marginally better fits than the radially anisotropic models. For the NFW and MOND models, none of the choices of the $\beta$ parameter can be preferred.

For the Newtonian mass-follows-light approach, we present two plots: in Fig. 20, the comparison is made for the inner region (interior to $\sim 3 R_{\mathrm{e}}$ ), and in Fig. 21 the comparison is made for the outer region (exterior to $\sim 3 R_{\mathrm{e}}$ ), except for two objects, NGC 4365 and NGC 4486, for which the exterior parts are beyond $1 R_{\mathrm{e}}$ and $0.35 R_{\mathrm{e}}$, respectively. One object (NGC 2768) is present only in Fig. 20 because the estimate made using the Jeans models for this galaxy is valid for the whole radial extent.

In the inner region, the only galaxy for which there is a significant discrepancy is NGC 4494 for which the Jeans models provided an anomalously low mass-to-light ratio; in the outer 
parts of this galaxy, there is agreement between the SPS and Jeans models, but, as already noted, either there is an increase of the stellar mass-to-light ratio of this galaxy by a factor of $\sim 4$ between the innermost and outermost radii, or DM is required (see Fig. 21). Only a detailed observational study of the stellar populations in NGC 4494 will clarify this issue.

With regard to the remaining early-type galaxies from our sample in the inner parts (Fig. 20), the predicted intermediate mass-to-light ratio based on the IP13 and BC models provide the best agreement with the values obtained using the Jeans models. For those galaxies with strong indications of massive dark halos (see Fig. 21), their inner parts can also be described with the higher stellar mass-to-light ratios based on the tested SPS models (the only exception is NGC 5846). Since a given SPS model must provide the estimate of the mass-to-light ratio at all radii, this is a strong indication of the existence of DM in the regions beyond $\sim 3 R_{\mathrm{e}}$.

Figures 20 and 21 thus suggest that there is no SPS model that can reconcile the dynamical mass-to-light ratios with the stellar component only and that galaxies NGC 1400, NGC 1407, NGC 3115, NGC 4278, NGC 4365, NGC 4486, and NGC 5846 require massive dark halos. Galaxies NGC 3377 and NGC 4494 also show the gradients in their mass-to-light ratios that are indicative of the existence of DM in them. Figures 20 and 21 thus suggest that there exists a value of the inner dynamical mass above which a massive dark halo in early-type galaxy can be expected: we estimate that this value based on Eq. (17), is $\log \left(M_{\text {dyn }} / M_{\odot}\right) \simeq 11.2\left(M_{\text {dyn }} \simeq 1.58 \times 10^{11} M_{\odot}\right)$. Future more precise measurements of the dynamical mass and the study of stellar populations will hopefully confirm (or rule out) the existence of such a value and provide a more precise estimate of the limiting value of the total dynamical mass in the case that it indeed exists.

For two galaxies below $M_{\text {dyn }} \simeq 1.58 \times 10^{11} M_{\odot}$, we only have a hint of the existence of DM (NGC 3377 and NGC 4494), and for one galaxy above the given limit (NGC 2768, which has a relatively high mass-to-light ratio), we have a hint of the lack of significant amount of DM. Only a detailed study of the stellar content in these objects provides a clear answer to the question of its contribution to their total dynamical mass. It is interesting to note that this critical mass, $M_{\text {dyn }} \simeq 1.58 \times 10^{11} M_{\odot}$, which appears to be close to the total mass within $R_{\mathrm{e}}$, mostly stellar, is close to the critical stellar mass beyond which Cattaneo et al. (2011) argue that most galaxies are built by mergers $\left(M_{\text {crit }}=\right.$ $10^{11} h^{-1} M_{\odot}=1.43 \times 10^{11} M_{\odot}$ for the value of the Hubble parameter we adopted in this paper). Since we have only two galaxies below the aforementioned critical mass, the conclusion regarding the separation of dynamical classes can only be tentative, and larger samples with more galaxies with lower masses will provide a stronger conclusion regarding this issue. Figures 20 and 21 show that the most massive galaxies from our sample belong to the class of slow rotators: these galaxies (NGC 1407, NGC 4365, NGC 4486, and NGC 5846) cannot be fit without significant amount of DM in their outer parts.

The MOND predictions for all three tested formulae are also presented in two plots: in Fig. 22, the comparison is again made for the inner region (interior to $\sim 3 R_{\mathrm{e}}$ ), and in Fig. 23 , the comparison is made for the outer region (exterior to $\sim 3 R_{\mathrm{e}}$ ) again, except for two objects, NGC 4365 and NGC 4486, for which the exterior parts are beyond $1 R_{\mathrm{e}}$ and $0.35 R_{\mathrm{e}}$, respectively). Figure 22 shows that one can see that MOND can describe the following two objects using a single mass-to-light ratio without splitting the analysis into the inner and outer parts: NGC 1400 and NGC 2768. As in the case of the Newtonian models presented above, the anomalously low $M / L$ values of the galaxy NGC 4494 cannot be reconciled with the SPS models. In general, the simple MOND model used with the IP13 SPS with Salpeter IMF models can provide a satisfactory prediction for the mass-to-light ratio in the inner parts of the galaxies in the sample (Fig. 22), where the stellar component dominates. Besides NGC 4494, the only exception is NGC 4365.

With regard to the investigation of $\mathrm{DM}$, the three tested MOND formulae all provide similar predictions for the massto-light ratios and, given the uncertainties, one cannot single out one formula that provides a better fit with respect to the remaining two. When one analyses the outer regions of the earlytype galaxies from the sample (Fig. 23), it becomes evident that six galaxies (NGC 1407, NGC 3115, NGC 4278, NGC 4365, NGC 4486, and NGC 5846) cannot be fit using the MOND approach regardless of the formula used. As in the previous case of the Newtonian modeling, a limiting value of the dynamical mass $\log \left(M_{\text {dyn }} / M_{\odot}\right) \simeq 11.2\left(M_{\text {dyn }} \simeq 1.58 \times 10^{11} M_{\odot}\right)$ separates two classes of early-type galaxies: the objects for which the dynamical mass is below this value, which can be fit using MOND, and those objects for which the dynamical mass is above this value, which require an additional DM halo. The future investigations using larger samples of galaxies (with better observational data) will probably provide more accurate answers about the existence of such a limit where MOND appears to have a breakdown. The firm conclusion coming as a result of modeling performed in the present paper is that there exists massive early-type galaxies with dynamical masses above $M_{\text {dyn }} \simeq 1.58 \times 10^{11} M_{\odot}$ for which a significant amount of DM is necessary even when using the MOND approach in all three tested approximations. Figure 23 shows that the most massive galaxies from our sample that belong to the class of slow rotators (NGC 1407, NGC 4365, NGC 4486, and NGC 5846) cannot be fit using MOND without significant amount of DM in their outer parts; moreover, NGC 4486 needs DM in MOND even in its inner part.

If one takes two examples of well-known, early-type galaxies from the literature that were mentioned in Sect. 1, one may test the aforementioned limit: NGC 5128 with a mass below the critical mass can be fit using MOND without any DM, whereas NGC 1399 with a mass above the critical mass requires additional DM (in both Newtonian and MOND dynamics).

Another important result of this paper can be seen in Fig. 24: it shows the dependence of the concentration parameter as a function of the virial mass, and it can be used to divide all the eleven galaxies in the sample into two classes. The shaded area represents the region determined by the mean relation expected from $\Lambda \mathrm{CDM}$ model for which the central line is given with

$c_{\mathrm{vir}}\left(M_{\mathrm{vir}}\right) \simeq 12\left(\frac{M_{\mathrm{vir}}}{10^{11} M_{\odot}}\right)^{-0.094}$,

where the limits are determined with $1 \sigma$ scatter (equal to 0.11 dex, see Napolitano et al. 2011).

Figure 24 indicates that the concentration parameter of only one galaxy (NGC 1407) is consistent with the $\Lambda \mathrm{CDM}$ simulations and that the galaxies tend to cluster into two classes: one class (Class 1, which consists of NGC 4278, NGC 3115, NGC 4365, NGC 4486, and NGC 5846) that is above the region of $\Lambda C D M$ halos (hatched region) and the other class (Class 2, which consists of NGC 3377, NGC 2768, NGC 1400, and NGC 4494) that is below the hatched region. The value of the concentration parameter, where the division occurs is $c_{\mathrm{vir}}^{\lim } \sim 7$. The galaxy NGC 1407 for which the concentration parameter is consistent with the $\Lambda \mathrm{CDM}$ simulations is clearly the example of an object with a significant amount of DM, and it cannot 


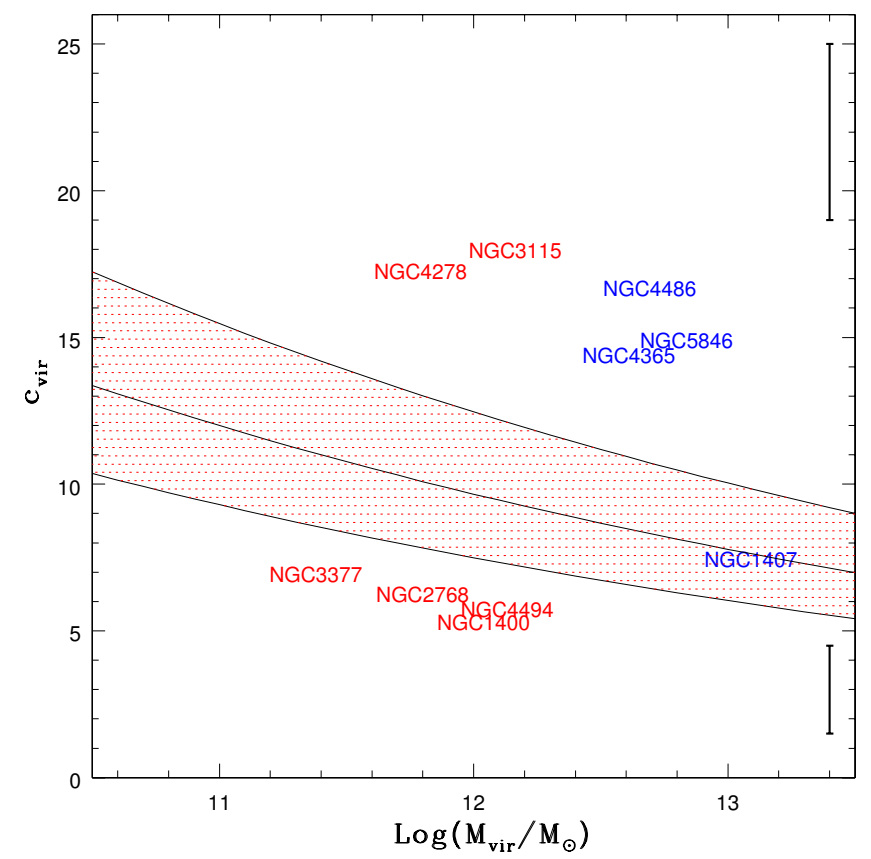

Fig. 24. Concentration parameter as a function of the virial mass (expressed in the solar units). The central line of the hatched region is given by Eq. (18), and its limits are determined with $1 \sigma$ scatter (see text for details). The vertical solid lines on the right hand side of the plot represent the typical error bars for the concentration parameter for two classes of galaxies: the lower line is for the objects below the hatched region, and the higher line is for the objects above the hatched region. The names of the slow (fast) rotators are plotted in blue (red).

be modeled in both approaches, Newtonian and MOND, without the inclusion of the invisible, dark component (see Figs. 21 and 23). Both classes of galaxies in the sample can be modeled with NFW halos of various normalizations.

The galaxies in Class 1 show a clear increase of the massto-light ratio beyond $\sim 3 R_{\mathrm{e}}$ which is indicative of the existence of DM (although NGC 3377, NGC 4494, and NGC 1400 from Class 2 also show the increase of the mass-to-light ratio). The galaxies belonging to Class 2 can be modeled without DM (or with its low content) in both Newtonian and MOND approaches. In general, the galaxies from Class 1 cannot be modeled with MOND without the inclusion of an additional, dark component. This result obtained on the sample of ten early-type galaxies confirms the ambiguity already noted in the literature: some objects appear to have low amounts of DM (or may even be completely devoid of the DM halos), and such galaxies can be also modeled in the MOND approach. On the other hand, another equally important class of galaxies appears to possess massive dark halos. The MOND approach cannot eliminate its influence, and thus, $\mathrm{DM}$ is required in such cases, which obviously introduces unwanted complications in the MOND theory. Examples of such galaxies are NGC 1407, NGC 3115, NGC 4365, NGC 4278, NGC 4486, and NGC 5846. For these galaxies, we could not find any MOND model that could provide a fit to the LOS observed velocity dispersion without DM. The existence of two classes of galaxies might be connected to the bimodality discussed by Napolitano et al. (2011) in which the authors suggest that "the less dramatic DM content found in lower-luminosity "ordinary" ellipticals suggests a bimodality in the halo properties which may be produced by divergent baryonic effects during their assembly histories".
Since we applied only spherical Jeans models in our analysis, one may ask how our conclusions are changed when we omit three galaxies that show flattening of their isophotes. When we omit these galaxies (NGC 2768, NGC 3377, and NGC 3115), the conclusions do not change. First, from Figs. 20-23, one sees that the established critical mass remains equal to $M_{\text {dyn }} \simeq 1.58 \times$ $10^{11} M_{\odot}$. Second, one can see in Fig. 24 that two galaxies remain below the hatched zone (NGC 1400 and NGC 4494), and four galaxies remain above it (NGC 4278, NGC 4365, NGC 4486, and NGC 5846), and therefore, the conclusions reached above remain unchanged.

Figure 24 indicates that the galaxies below the hatched region (NGC 1400, NGC 2768, NGC 3377 and NGC 4494), or those with low concentration parameters relative to $\Lambda \mathrm{CDM}$ halos of the same virial mass $\left(c_{\mathrm{vir}} \sim 5-7\right)$ all have the $\gamma$-parameter related to the surface density profiles of their GCs in their outer regions in a narrow range: $1.70 \leq \gamma \leq 2.20$. However, the galaxy with the highest concentration parameter, NGC 3115 $\left(c_{\mathrm{vir}}=18.07\right)$ has the highest value of the $\gamma$-parameter: $\gamma=4.17$.

Another interesting conclusion related to the isophote shapes can be reached from Fig. 24. All the galaxies below the hatched zone (except NGC 1400) belong to the disky population, and all the galaxies above it (except NGC 3115) are boxy galaxies. With regard to the profile types below the hatched zone, we have two galaxies with a power law and one with core profile, while we have three objects above it with a core and one with a power law profile, so firm conclusions cannot be reached at this stage.

\section{Conclusions}

The dynamics of the sample of ten massive nearby early-type galaxies was studied using the spherical Jeans equation to establish the importance of DM in such objects. We used GCs as a tracer of the potential of the galaxies. To infer the existence of DM, we used the Newtonian (mass-follows-light and stars + NFW DM) models and MOND models to calculate the mass-tolight ratios, which can then be compared with the predictions of various SPS models based on the stellar matter.

In Table 9, we list only the most important conclusions for five sets of models: Newtonian models without DM for the inner parts, Newtonian models without DM for the outer parts, stars + NFW DM models for the whole galaxies, MOND models without DM for the inner parts, and MOND models without DM for the outer parts. The detailed presentation of the conclusions is given below. All three MOND models for the transition from the MOND to Newtonian regimes are presented together in Table 9 (Cols. 5 and 6).

Our conclusions are as follows.

1. The kinematics of the sample of ten early-type galaxies based on the observed GCs, which are taken from the SLUGGS database, were studied, and it was found that the velocity dispersion remains approximately constant beyond $\sim 1-2 R_{\mathrm{e}}$ in all cases where several observed points are available. We also extracted the kurtotic parameter, $s_{4}$, for all the galaxies in the sample and found that it is consistent with zero or moderately negative in many cases, implying small tangential anisotropies. Thus, we tested cases that were isotropic, were mildly tangential anisotropic, and had positive values of anisotropies based on the numerical simulations.

2. We solved the Jeans equation for two Newtonian models: mass-follows-light and stars + NFW DM models both in the spherical approximation. We found that only one early-type 
Table 9. Summary of the conclusions.

\begin{tabular}{|c|c|c|c|c|c|}
\hline $\begin{array}{c}\text { Name } \\
\text { (1) }\end{array}$ & $\begin{array}{l}\text { Newtonian-noDM } \\
\text { (int) } \\
\text { (2) }\end{array}$ & $\begin{array}{c}\text { Newtonian-noDM } \\
\text { (out) } \\
\text { (3) }\end{array}$ & $\begin{array}{c}\text { Newtonian-DM } \\
\text { (int and out) } \\
\text { (4) }\end{array}$ & $\begin{array}{l}\text { MOND-noDM } \\
\text { (int) } \\
\text { (5) }\end{array}$ & $\begin{array}{l}\text { MOND-noDM } \\
\text { (out) } \\
\text { (6) }\end{array}$ \\
\hline NGC 1400 & $\sqrt{ }$ & - & $\sqrt{ }$ & $\sqrt{ }$ & $\sqrt{ }$ \\
\hline NGC 1407 & $\sqrt{ }$ & - & $\sqrt{ }$ & $\sqrt{ }$ & - \\
\hline NGC 2768 & $\sim$ & $\sim$ & $\sqrt{ }$ & $\sqrt{ }$ & $\sqrt{ }$ \\
\hline NGC 3115 & - & - & $\sqrt{ }$ & $\sqrt{ }$ & - \\
\hline NGC 3377 & $\sqrt{ }$ & - & $\sqrt{ }$ & $\sqrt{ }$ & $\sqrt{ }$ \\
\hline NGC 4278 & - & - & $\sqrt{ }$ & $\sqrt{ }$ & - \\
\hline NGC 4365 & - & - & $\sqrt{ }$ & $\sqrt{ }$ & - \\
\hline NGC 4486 & - & - & $\sqrt{ }$ & - & - \\
\hline NGC 4494 & $\sqrt{ }$ & $\sqrt{ }-$ & $\sqrt{ }$ & $\sqrt{ }$ & $\sqrt{ }$ \\
\hline NGC 5846 & $\sqrt{ }$ & - & $\sqrt{ }$ & $\sqrt{ }$ & - \\
\hline
\end{tabular}

Notes. Column (1): name of the galaxy. Column (2): Newtonian model without DM for the internal part (interior to $\sim 3 R_{\mathrm{e}}$ ). Column (3): Newtonian model without DM for the exterior part (exterior to $\sim 3 R_{\mathrm{e}}$ ). Column (4): stars + NFW DM model (for the whole galaxy). Column (5): MOND models without DM for the internal part (interior to $\sim 3 R_{\mathrm{e}}$ ). Column (6): MOND models without DM for the exterior part (exterior to $\sim 3 R_{\mathrm{e}}$ ). Here, the sign " $\sqrt{ }$ " denotes that a given model can provide a successful fit to the observed data. The sign "-" denotes that the given model cannot provide a successful fit to the observed data. The sign " " for galaxy NGC 2768 denotes that this galaxy can be fit with a constant mass-to-light ratio, but the value obtained is marginally higher than that predicted by SPS models. The combination of signs (" $\sqrt{ }-$ ") for NGC 4494 denotes that the mass-to-light ratio obtained for this galaxy in its outer parts is consistent with the value obtained using SPS models. Because of the increase of the mass-to-light ratio in its outer part, however one cannot exclude the existence of DM (see text for details).

galaxy (for which we used a single value of the $\gamma$ parameter in the power law) can be modeled with a single value of the constant mass-to-light ratio that is approximately consistent with the value of the stellar component, NGC 2768, showing the lack of significant amount of DM. Three more galaxies (NGC 1400, NGC 3377, and NGC 4494) show an increase of the total mass-to-light ratio with radius between the innermost and outermost radii, which suggests the existence of DM in them. The object NGC 4486 is the only galaxy that needs significant amount of DM in its inner region. The case of NGC 2768 is complex because a high mass-to-light ratio obtained in the inner region suggests the existence of DM even in the innermost parts of the galaxy $\left(r \lessgtr 1 R_{\mathrm{e}}\right)$ assuming radial anisotropies. The remaining five galaxies require a significant amount of DM beyond $\sim 2-3 R_{\mathrm{e}}$ to explain their dynamics that are inferred using GCs. The objects NGC 1407, NGC 4278, NGC $4365^{\circ}$, and NGC 3115 require DM beyond $\sim 3 R_{\mathrm{e}}$, whereas DM becomes important in NGC 5846 beyond $\sim 2 R_{\mathrm{e}}$ and NGC 4486 requires $\mathrm{DM}$ in its inner parts (interior to $\sim 0.35 R_{\mathrm{e}}$ ), as shown above. The largest estimated mass-to-light ratios were found in NGC 5846 for which $64.2<M / L_{B}<127.4$ beyond $\sim 6 R_{\mathrm{e}}$ was established. The lowest mass-to-light ratio was found in NGC 4494 for which we find $2.6<M / L_{B}<5.7$. All slow rotators in our sample require a significant amount of DM in their outer parts. The NFW models provided the best fits for all the galaxies in the sample. The inferred density $\rho_{\mathrm{s}}$ varied from low values found in NGC $4494\left(0.0015<\rho_{\mathrm{s}}<0.0060 M_{\odot} \mathrm{pc}^{-3}\right.$; the same lower value was also found for NGC 1400) to higher values, $0.0600<\rho_{\mathrm{s}}<0.1200 M_{\odot} \mathrm{pc}^{-3}$, found for NGC 3115. Our results are in good agreement for those galaxies for which the estimates of the mass-to-light ratio in the outer parts exist in the literature.

3. We also solved the Jeans equation in the spherical approximation for three different MOND models: we tested standard, simple and toy models. To model the LOS observed velocity dispersion points of the galaxies from the sample,

\footnotetext{
8 Because of its large effective radius used in this paper this galaxy requires a significant amount of DM beyond one effective radius.
}

the standard model required the largest mass-to-light ratio while the toy MOND model required the smallest. We found that the following galaxies could be modeled with a mass-tolight ratio consistent with the no dark-matter hypothesis by assuming the values of the mass-to-light ratios that are consistent with the stellar mass only: NGC 1400, NGC 2768, NGC 3377, and NGC 4494. The case of NGC 4486 is peculiar. This is the only galaxy in our sample that requires DM in MOND in its inner region (interior to $\left.\sim 0.35 R_{\mathrm{e}}\right)^{9}$. The following galaxies require DM even in the MOND approach in their outer parts. The objects NGC 1407, NGC 4278, NGC 5846, and NGC 3115 require DM beyond $\sim 3 R_{\mathrm{e}}$, and NGC 4365 and NGC 4486 require DM beyond $\sim 1 R_{\mathrm{e}}(\sim 20.7 \mathrm{kpc})$ and $\sim 0.35 R_{\mathrm{e}}(\sim 15 \mathrm{kpc})$, respectively. The general conclusion regarding the MOND modeling of the early-type galaxies in the sample is that some galaxies can be fit with the visible matter only; in some cases, the additional dark component must be added to obtain successful fits of the LOS observed velocity dispersion. This obviously diminishes the appeal of MOND based on one free parameter only (mass-to-light ratio in this case). Whether these problems are a consequence of a breakdown of MOND for certain objects "remains unclear" as Famaey \& McGaugh (2012) warn. From our analysis, one can conclude that the MOND approach cannot provide a successful fit for the galaxies that are slow rotators. We speculate that the dynamical mass $M_{\text {dyn }} \simeq 1.58 \times 10^{11} M_{\odot}$ separates two classes of early-type galaxies (based on our sample that includes only two galaxies below and eight galaxies above the limit). The objects for which the established dynamical mass is below this value can be fit by using MOND with the stellar component only, whereas those objects for which the dynamical mass is above this value require an additional, dark halo. The examples of two well-known objects found in the literature (NGC 5128, as the galaxy below this limit, and NGC 1399, as the galaxy above it) support this hypothesis.

9 As shown above, there is a strong indication for NGC 4365 that DM is required in MOND, even interior to one effective radius, because only the tangentially anisotropic toy MOND model can marginally fit the kinematics of GCs there. 
This critical mass, which represents a total stellar mass, is close to the critical mass beyond which Cattaneo et al. (2011) argue that most galaxies are built by mergers.

4. We compared our result from the Jeans modeling with the estimates of the stellar mass-to-light ratios coming from the following SPS models with various IMFs: BC models (based on the Salpeter IMF), PEGASE models (based on the Salpeter IMF), IP13 exponential SFH, and disc galaxy models (based on the Kroupa and Salpeter IMFs), and CvD12 models (with IMF variation and a fixed MW Kroupa IMF). We found that the galaxies in our sample are best described with the IP13 Salpeter models for the Newtonian models. In the case of the MOND models, the IP13 SPS models with the Kroupa IMF can best describe the galaxies in our sample using the simple MOND formula.

5. We calculated the virial mass and the virial concentration index (of the DM) for all the galaxies in the sample. We found that the value of the concentration index of only one galaxy (NGC 1407) agrees with the $\Lambda$ CDM simulations, and we note that the existence of two classes of objects emerges. For one class, the concentration index is above the value predicted by the $\Lambda \mathrm{CDM}$ simulations (NGC 4278, NGC 4486, NGC 4365, NGC 3115, and NGC 5846): these galaxies show the increase of the mass-to-light ratio in their outer parts and, thus, require DM in Newtonian approach. They also require DM in the various MOND models and, for five of them, (NGC 3115, NGC 4278, NGC 4365, NGC 4486, and NGC 5846) with NGC 1407, no MOND model could be found that provides a fit to the LOS observed velocity dispersion without DM. For the other class of galaxies, which include NGC 3377, NGC 2768, NGC 4494, and NGC 1400, the concentration index is below the value predicted by the $\Lambda C D M$ simulations. The values of the mass-to-light ratio related to them is consistent with the hypothesis with no DM or that with the small amount of DM. The galaxies from this class can be fit using the MOND models without invoking the dark component. If one wants to generalize the conclusions based on the results obtained using the virial concentration index, one can generally notice that, both Newtonian and MOND models must include its contribution whenever there is a need of a significant amount of DM in a certain galaxy: when such a case is encountered, none of the three different tested MOND models proved to be superior and capable to provide a successful fit where the other tested MOND models failed. Whereas fast rotators can have either the smallest or highest values of the concentration parameter that is not consistent with the predictions of the $\Lambda$ CDM simulations, the galaxies that are slow rotators have values of concentration parameters that either agree with the simulations (NGC 1407) or are higher.

6. It remains difficult to generalize the conclusion about the existence and importance of DM in early-type galaxies. Only new, carefully conceived observational campaigns (SLUGGS is a valuable accomplishment in this respect) with improved dynamical and stellar population modeling can bring progress to our understanding of the content of early-type galaxies. The analysis of the galaxies in the sample analyzed above proves that a variety of possibilities is present - from galaxies, which DM is not necessary (or at least its contribution is minor) for the explanation of their dynamics, to galaxies, which DM dominates luminous matter in the outer parts. Our present analysis, however, strongly suggests that DM in early-type galaxies is not dynamically dominant when the values are less than $\sim 3 R_{\mathrm{e}}$, which indicates a diffuse DM distribution (see e.g. Matteucci 2012). This agrees with our earlier findings based on the analysis of their long-slit spectra (Samurović \& Danziger 2005; Samurović 2007). Beyond $\sim 3 R_{\mathrm{e}}$, DM may or may not dominate luminous matter, and bigger samples of observed earlytype galaxies, which will provide more objects, will help us to firmly establish whether the apparent lack of DM in some early-type galaxies is an anomaly or the rule.

Acknowledgements. This work was supported by the Ministry of Education, Science and Technological Development of the Republic of Serbia through the project No. 176021, "Visible and Invisible Matter in Nearby Galaxies: Theory and Observations". The author expresses his gratitude to the referee, Dr. Gary Mamon, on the detailed report and numerous useful suggestions and corrections that significantly improved the quality of the manuscript. The author also thanks the editor for the help. This research made use of the NASA/IPAC Extragalactic Database (NED), which is operated by the Jet Propulsion Laboratory, California Institute of Technology, under contract with the National Aeronautics and Space Administration. We acknowledge the usage of the HyperLeda database (http: //leda.univ-lyon $1 . \mathrm{fr}$ ).

\section{References}

Arnold, J. A., Romanowsky, A. J., Brodie, J. P., et al. 2014, ApJ, 791, 80 Auger, M. W., Treu, T., Bolton, A. S., et al. 2009, ApJ, 705, 1099

Bekenstein, J. 2004, Phys. Rev. D, 70, 083509

Bell, E. F., \& de Jong, R. S. 2001, ApJ, 550, 212

Bender, R., Surma, P., Döbereiner, S., Möllenhoff, C., \& Madejsky, R. 1989, A\&A, 217, 35

Bertin, G., Ciotti, L., \& Del Principe, L. 2002, A\&A, 386, 149

Bertola, F., Pizzella, A., Persic, M., \& Salucci, P. 1993, ApJ, 416, L45

Binney, J. J., \& Mamon, G. 1982, MNRAS, 200, 361

Binney, J. J., \& Tremaine, S. 2008, Galactic Dynamics, 2d edn. (Princeton: Princeton Univ. Press)

Bruzual, G., \& Charlot, S. 2003, MNRAS, 344, 1000 (BC)

Cappellari, M., Bacon, R., Bureau, M., et al. 2006, MNRAS, 366, 1126 Cappellari, M., Scott, N., Alatalo, K., et al. 2013a, MNRAS, 432, 1709 Cappellari, M., McDermid, R. M., Alatalo, K., et al. 2013b, MNRAS, 432, 1862 Carlberg, R. G., Yee, H. K. C., \& Ellingson, E. 1997, 478, 462

Casuso, E., Vazdekis, A., Peletier, R. F., \& Beckman, J. E. 1996, ApJ, 458, 533

Cattaneo, A., Mamon, G. A., Warnick, K., \& Knebe, A. 2011, A\&A, 533, A5

Coccato, L., Gerhard, O., Arnaboldi, M., et al. 2009, MNRAS, 394, 1249

Conroy, C., Prada, F., Newman, J. A., et al. 2007, ApJ, 654, 153

Conroy, C., \& van Dokkum, P. G. 2012, ApJ, 760, 71 (CvD12)

Courteau, S., Cappellari, M., de Jong, R. S., et al. 2014, Rev. Mod. Phys., 86, 47 Crocker, A. F., Bureau, M., Young, L. M., \& Combes, F. 2008, MNRAS, 386, 1811

da Costa, L. N., Willmer, C. N. A., Pellegrini, P. S., et al. 1998, AJ, 116, 1 de Lorenzi, F., Gerhard, O., Coccato, L., et al. 2009, MNRAS, 395, 76

de Vaucouleurs, G., de Vaucouleurs, A. Corwin, H. G. Jr., et al. 1991, 3rd Reference Catalogue of Bright Galaxies (New York: Springer-Verlag)

Deason, A. J., Belokurov, V., Evans, N. W., \& McCarthy, I. G. 2012, ApJ, 748, 2 Dekel, A., Stoehl, F., Mamon, G. A., et al. 2005, Nature, 437, 707

Diehl, S., \& Statler, T. S. 2007, ApJ, 668, 150

Duncan, M. J., \& Wheeler, J. C. 1980, ApJ, 237, L27

Emsellem, E., Dejonghe, H., \& Bacon, R. 1999, MNRAS, 303, 495

Emsellem, E., Cappellari, M., Krajnović, D., et al. 2007, MNRAS, 379, 401

Famaey, B., \& Binney, J. 2005, MNRAS, 363, 603

Famaey, B., \& McGaugh, S. S. 2012, Liv. Rev. Relativity, 15, 10

Famaey, B., Gentile, G., Bruneton, J.-P., \& Zhao, H. S. 2007a, Phys. Rev. D, 75, 3002

Famaey, B., Bruneton, J.-P., \& Zhao, H.S, 2007b, MNRAS, 377, L79

Faure, C., Kneib, J.-P., Covone, G., et al. 2008, ApJSS, 176, 19

Fioc, M., \& Rocca-Volmerange, B. 1997, A\&A, 326, 550

Garcia, A. M. 1993, A\&AS, 100, 47

Gerhard, O. 1993, MNRAS, 265, 213

Gnedin, O. Y., Brown, W. R., Geller, M. J., \& Kenyon, S. J. 2010, ApJ, 720, L108

Graham, A. W., Erwin, P., Trujillo, I., \& Ramos, A. A. 2003, AJ, 125, 2951

Gunn, J. E., \& Gott, J. R. 1972, ApJ, 176, 1

Hernquist, L. 1990, ApJ, 356, 359

Hu, J. 2008, MNRAS, 386, 2242

Humphrey, P. J., Buote, D. A., Gastaldello, F., et al. 2006, ApJ, 646, 899

Humphrey, P. J., Buote, D. A., Canizares, C. R., Fabian, A. C., \& Miller, J. M. 2011, ApJ, 729, 53 
Into, T., \& Portinari, L. 2013, MNRAS, 430, 2715 (IP13)

Jarrett, T. H., Chester, T., Cutri, R., Schneider, S. E., \& Huchra, J. P. 2003, AJ, 125,525

Joanes, D. N., \& Gill, C. A. 1998, The Statistician, 47, 183

Katgert, P., Biviano, A., \& Mazure, A. 2004, ApJ, 600, 657

Kleinheinrich, M., Schneider, P., Rix, H.-W., et al. 2006, A\&A, 455, 441

Kormendy, J., Fisher, D. B., Cornell, M. E., \& Bender, R. 2009, ApJSS, 182, 216

Krajnović, D., Alatalo, K., Blitz, L., et al. 2013, MNRAS, 432, 1768

Kroupa, P. 2001, MNRAS, 322, 231

Lauer, T. R., Faber, S. M., Gebhardt, K., et al. 2005, AJ, 129, 2138

Lauer, T. R., Gebhardt, K., Faber, S. M., et al. 2007, ApJ, 664, 226

Lemze, D., Postman, M., Genel, S., et al. 2013, ApJ, 776, 91

Łokas, E. L. 2003, MNRAS, 333, 697

Łokas, E. L. 2008, ApJ, 680, L101

Łokas, E. L., \& Mamon, G. A. 2003, 343, 401

Mamon, G. A., \& Lokas, E. L. 2005, MNRAS, 363, 705

Mastropietro, C., Moore, B., Mayer, L., et al. 2005, MNRAS, 364, 607

Mathews, W. G., \& Brighenti, F. 2003, ARA\&A, 41, 191

Matteucci, F. 2012, Chemical Evolution of Galaxies (Berlin Heidelberg: Springer-Verlag)

Mei, S., Blakeslee, J. P., Côté, P., et al. 2007, ApJ, 655, 144

Merritt, D. 1987, ApJ, 313, 121

Milgrom, M. 1983, ApJ, 270, 365

Milgrom, M. 2012, Phys. Rev. Lett. 109, id. 131101

Milgrom, M., \& Sanders, R. H. 2003, ApJ, 599, L25

More, S., van den Bosch, F. C., Cacciato, M., et al. 2011, MNRAS, 410, 210

Morganti, L., Gerhard, O., Coccato, L., Martinez-Valpuesta, I., \& Arnaboldi, M. 2013, MNRAS, 432, 3350

Napolitano, N. R., Romanowsky, A. J., Coccato, L., et al. 2009, MNRAS, 393, 329

Napolitano, N. R., Romanowsky, A. J., Capaccioli, M., et al. 2011, MNRAS, 411,2035

Napolitano, N. R., Pota, V., Romanowsky, A. J., et al. 2014, MNRAS, 439, 659

Navarro, J. F., Frenk, C. S., \& White, S. D. M. 1997, ApJ, 490, 493

Nieto, J.-L., Bender, R., Arnaud, J., \& Surma, P. 1991, A\&A, 244, L25

Pota, V., Forbes, D. A. Romanowsky, A. J., et al. 2013, MNRAS, 428, 389 (P13)
Prugniel, P., \& Simien, F. 1997, A\&A, 321, 111

Rest, A., van den Bosch, F. C., \& Jaffe, W. 2001, AJ, 121, 2431

Richardson, T., \& Fairbairn, M. 2013, MNRAS, 432, 3361

Richtler, T., Schuberth, Y., Hilker, M., et al. 2008, A\&A, 478, L23

Richtler, T., Famaey, B., Gentile, G., \& Schuberth, Y. 2011, A\&A, 531, A100

Rix, H.-W., de Zeeuw, P. T., Cretton, N., van der Marel, R. P., \& Carollo, C. M. 1997, ApJ, 488, 702

Romanowsky, A. J., Douglas, N. G., Arnaboldi, M., et al. 2003, Science, 5640, 1696

Romanowsky A. J., Strader J., Spitler L. R., et al. 2009, AJ, 137, 4956

Salinas, R., Richtler, T., Bassino, L. P., Romanowsky, A. J., \& Schuberth, Y. 2012, A\&A, 538, A87

Samurović, S. 2007, Dark Matter in Elliptical Galaxies, Publications of the Astronomical Observatory of Belgrade, 81

Samurović, S. 2010, A\&A, 514, A95

Samurović, S. 2012, A\&A, 541, A138

Samurović, S., \& Danziger, I. J. 2005, MNRAS, 363, 769

Samurović, S., \& Danziger, I. J. 2006, A\&A, 458, 79

Samurović, S., \& Ćirković, M. M. 2008, A\&A, 488, 873

Samurović, S., \& Lalović, A. 2011, A\&A, 531, A82

Sandage, A., \& Bedke, J. 1994, The Carnegie Atlas of Galaxies, Volumes I, II (Carnegie Institution of Washington Publ.)

Sanders, R. H., \& McGaugh, S. 2002, ARA\&A, 40, 263

Saxton, C. J., \& Ferreras, I. 2010, MNRAS, 405, 77

Schlegel, D. J., Finkbeiner, D. P., \& Davis, M. 1998, ApJ, 500, 525

Schuberth, Y., Richtler, T., Hilker, M., et al. 2012, A\&A, 544, A115

Schwarzschild, M. 1979, ApJ, 232, 236

Spolaor, M., Forbes, D. A., Hau, G. K. T., Proctor, R. N., \& Brough, S. 2008, MNRAS, 385, 667

Strader, J., Romanowsky, A. J., Brodie, J. P., et al. 2011, ApJSS, 197, 33

Tiret, O., Combes, F., Angus, G. W., Famaey, B., \& Zhao, H. S. 2007, A\&A 476, L1

Tonry, J. L. 1983, ApJ, 266, 58

Tonry, J. L., Dressler, A., Blakeslee, J. P., et al. 2001, ApJ, 546, 681

Tortora, C., Napolitano, N. R., Romanowsky, A. J., \& Jetzer, P. 2010, ApJ, 721, L1

Wojtak, R., \& Mamon, G. 2013, MNRAS, 428, 2407 